\title{
MASTER MASTER
}

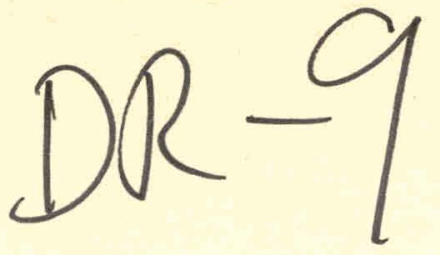

Kinetic Transport Properties of a Bumpy Torus with Finite Radial Ambipolar Field

\author{
D. A. Spong \\ E. G. Harris \\ C. L. Hedrick
}




\section{DISCLAIMER}

This report was prepared as an account of work sponsored by an agency of the United States Government. Neither the United States Government nor any agency Thereof, nor any of their employees, makes any warranty, express or implied, or assumes any legal liability or responsibility for the accuracy, completeness, or usefulness of any information, apparatus, product, or process disclosed, or represents that its use would not infringe privately owned rights. Reference herein to any specific commercial product, process, or service by trade name, trademark, manufacturer, or otherwise does not necessarily constitute or imply its endorsement, recommendation, or favoring by the United States Government or any agency thereof. The views and opinions of authors expressed herein do not necessarily state or reflect those of the United States Government or any agency thereof. 


\section{DISCLAIMER}

Portions of this document may be illegible in electronic image products. Images are produced from the best available original document. 
Printed in the United States of America. Available from National Terhnir:al Informalion Service

U.S. Department of Commerce

5285 Port Royal Road, Springfield, Virginia 22161

Price: Printed Copy $\$ 6.50$; Microfiche $\$ 3.00$

This report was prepared as an account of work sponsored by an agency of the United States Government. Neither the United States Government nor any agency thereof, nor any of their employees, contractors, subcontractors, or their employees, makes any warranty, express or implied, nor assumes any legal liability or responsibility for any third party's use or the results of such use of any information, apparatus, product or process disclosed in this report, nor represents that its use by such third party would not infringe privately owned rights. 
ORNL/TM-6215

Dist. Category UC-20g

Contract No. W-7405-eng-26

FUSION ENERGY DIVISION

KINETIC TRANSPORT PROPERTIES OF A BUMPY TORUS

WITH FINITE RADIAL AMBIPOLAR FIELD

D. A. Spong, E. G. Harris, and C. L. Hedrick

Date Published: April 1978

NOTICE This document contains information of a preliminary nature.

It is subject to revision or correction and therefore does not represent a final report.

Prepared by the OAK RIDGE NATIONAL LABORATORY Oak Ridge, Tennessee 37830 operated by UNION CARBIDE CORPORATION for the DEPARTMENT OF ENERGY
This report was prepored an secount of work spansored by the United States Government. Neither the United States nor the United States Departmene of Energ, nor any of their employees, nor any of their contractors, subcontractors, of their employees, makes any warranty, express or implied, or assumes any lega liability or responsibility for the aceuracy, completerese or usefulness of any information, apparatus, product or process disclosed, or represents that its use would not infrimge privately owned rights. 


\section{THIS PAGE}

\section{WAS INTENTIONALLY \\ LEFT BLANK}


CONTENTS

FIGURES

ABSTRACT . . . . . . . . . . . . . . . . . . . . vii

I. INTRODUCTION . . . . . . . . . . . . . . . . . I

II. BOUNCE-AVERAGED DRIFT KINETIC EQUATION . . . . . . . . . 5

A. Drift and Acceleration Terms . . . . . . . . . 5

B. Collision Operator . . . . . . . . . . . . . . 9

III. TREATMENT OF CONFIGURATION SPACE DEPENDENCE . . . . . . . 17

A. Expansion of Theta Dependence . . . . . . . . . . 17

B. Loca1 Approximation to Radial Dependence . . . . . . . 20

C. Expressions for Transport Coefficients... . . . . . 21

IV. PARTICLE ORBIT MODEL . . . . . . . . . . . . . . 25

A. Bounce-Averaged Poloidal Drift Frequency . . . . . . 25

B. Bounce-Averaged Vertical Drift . . . . . . . . 36

V. NUMERICAL SOLUTION PROCEDURES AND RESULTS . . . . . . . . 41

A. Functional Expansion Method . . . . . . . . . . 42

B. Finite Difference (ADI) Method ............ 55

C. Range of Validity for Local Approximation . . . . . . 62

VI. PARTICLE AND ENERGY LIFETIME ESTIMATES . . . . . . . . . 65

VII. SUMMARY AND CONCLUSIONS . . . . . . . . . . . . . 69

APPENDIX A. PRECESSIONAL DRIFT FREQUENCY MATRIX ELEMENTS • • • 71

APPENDIX B. LANDAU COLLISION OPERATOR MATRIX ELEMENTS . • • • . 75

APPENDIX C. VARIATIONAL PRINCIPLE FOR TRANSPORT
COEFFICIENTS . . . . . . . . . . . . . . . . . 87

REFERENCES ........................... 91 


\section{THIS PAGE}

\section{WAS INTENTIONALLY LEFT BLANK}




\section{FIGURES}

\section{Figure}

1 Toroidal coordinate system and single particle drifts . . .

2 Poloidal drift frequency due to curvature and gradients in a bumpy cylinder field $(\vec{E} \times \vec{B}$ drifts are not included) $\mathrm{vs} v / \mathrm{v}_{\mathrm{th}}$ and $\zeta(=\cos x) \ldots . . .$.

3 Contours where the poloidal drift frequency goes to zero vs $v_{\perp} / v_{t h}$ and $v_{\|} / v_{t h}$ as a function of. the ambipolar potential

4 Dimensionless poloidal drift frequency function $\Omega^{\prime} / \mathrm{c}$ vs $\zeta$ (solid line) and approximate fit (dashed line) for EB'T-I parameters . . . . . . . . . .

5(a) Comparison of bounce-averaged vertical drift $\left\langle v_{y}\right\rangle$ (solid line) with the same drift before bounce $y$ averaging (dashed line)... . . . . . . . . . 38

5(b) Approximate fit (dashed line) to bounce-averaged vertical drift $\left\langle\mathrm{v}_{\mathrm{y}}\right\rangle$ (solid line) . . . . . . . . .

6 Convergence properties of functional representation method for $D_{n}$ transport coefficient vs $\nu_{0} / \Omega_{0}$ for $\mathrm{e} \phi_{\mathrm{o}} / \mathrm{kT}=0$

7 Convergence properties of functional representation method for $D_{n}$ transport coefficient vs $\nu_{0} / \Omega_{0}$ for $\mathrm{e} \phi_{0} / \mathrm{kT}=1$. . . . . . . . . . . . . . . . . . . .

$8 \quad t_{s n}^{\prime}$ distribution function $v s \zeta$ and $v / v_{\text {th }}$ for e $\phi_{0} / k T=0,0.2,0.4,0.6$, and $\nu_{0} / \Omega_{0}=0.1 \ldots . . .$.

9 (a) $D_{n}^{\prime}$ bumpy torus neoclassical transport coefficients vs $\nu_{0} / \Omega_{0}$ and as a function of the ambipolar potential ........................

9(b) $D_{\mathrm{T}}^{\prime}$ bumpy torus neoclassical transport coefficients vs $\nu_{0} / \Omega_{0}$ and as a function of the ambipolar potential . . . . . . . . . . . . . . . . .

9 (c) $K_{n}^{\prime}$ bumpy torus neoclassical transport coefficients vs $\nu_{0} / \Omega_{0}$ and as a function of the ambipolar potential . . . . . . . . . . . . . . . .

9 (d) $K_{T}^{\prime}$ bumpy torus neoclassical transport coefficients vs $\nu_{0} / \Omega_{0}$ and as a function of the ambipolar potential . . . . . . . . . . . . . . . . . 


\section{Figure}

10 Convergence properties of ADI finite difference solution of Eq. (93) using the constant coefficient model collision operator vs the damping factor $\alpha_{s} . . . .457$

11 Comparison between functional expansion and ADI finite difference solutions for the four transport coefficients vs e $\phi_{0} / k T$ and at $\nu_{0} / \Omega_{0}=1 . . . \cdot . . . .559$

12 Dependence of $\mathrm{D}_{\mathrm{n}}$ on $\mathrm{e} \phi_{\mathrm{o}} / \mathrm{kT}$ for positive and negative values at $\nu_{0} / \Omega_{0}=0.1$ (constant coefficient collision operator is used here) . . . . . . . . . 60

$13 \mathrm{f}_{\mathrm{s} n}^{\prime}$ distribution function $v s \zeta$ and $v / v_{\text {th }}$ for $\mathrm{e} \phi_{\mathrm{o}} / \mathrm{kT}=-U .4$ and $v_{\mathrm{o}} / \Omega_{\mathrm{o}}=0.1 \ldots . . . . . . . . .61$

14 Upper limit un $D_{11}^{\prime}$ for validity of the local approximation as indicated by Eq. (100) . . . . . . . 64

15 Neoclassical particle and energy lifetimes (msec) as a function of collisionality $\left(\nu_{0} / \Omega_{0}\right)$ and ambipolar field $\left(e \phi_{o} / \mathrm{kT}\right)$ for EBT-I parameters . . . . . 67 
Bumpy torus neoclassical transport coefficients have been calculated including finite values of the radial ambipolar field. These are obtained by solving a bounce-averaged drift kinetic equation in a local approximation for perturbations in the distribution function (away from a stationary Maxwellian) caused by toroidicity and radial gradients in plasma density, temperature, and potential. Particle and energy fluxes along with the associated transport coefficients are then calculated by taking appropriate moments of the distribution function. Particle orbits are treated by breaking them up into a vertical drift component (due to toroidicity) and a $\theta$ precessional drift (as a result of $\vec{E} \times \vec{B}$ and drifts due to the bumpy toroidal field). The kinetic equation has been solved using both a functional expansion method and finite difference techniques [Alternating-Direction-Implicit (ADI)]. The resulting transport coefficients exhibit a strong dependence on the ambipolar electric field and plasma collisionality. In the large electric field limit, our results are in close agreement with the earlier work of Kovrizhnykh. 1 


\section{INTRODUCTION}

The transport properties of high temperature plasmas in bumpy torus confinement systems have recently become of significant interest with respect to the interpretation of results obtained on the EBT experiment. ${ }^{2}$ Comparisons between the measured plasma parameters and simple neoclassical point models ${ }^{3}$ have indicated that the observed gross energy lifetimes could be largely accounted for by collisional transport processes. A primary motivation for developing an accurate bumpy torus transport model is that it is expected to represent an upper bound on the possible plasma containment in such a device. Continuing comparison of experimental results agalnst this point of reference indicates the extent to which nonclassical or anomalous transport mechanisms may be operative.

While extensive work has been done in recent years on neoclassical transport in axisymmetric devices, ${ }^{4,5}$ relatively little consideration has been given to nonaxisymmetric geometries. ${ }^{6}$ A unique feature of transport in the latter case is that electron and ion diffusion coefficients are not automatically equal and consequently an ambipolar field develops to maintain quasi-neutrality. This field acts to modify transport rates both by its direct influence through the cross-field mobility (electrostatic confinement) and by its effect on the centering of orbits (thus changing the step size and transport coefficients of one species relative to the other). Transport coefficients have been calculated ${ }^{1}$ using a Landau collision operator for the bumpy torus in the limit where the ambipolar field is large relative to the plasma thermal energy $(e \phi / k T$ $>1)$. Also, transport rates were given ${ }^{7}$ for a bumpy torus (and stellarator) using a Krook collision operator (most appropriate to weakly ionized gases) under certain limited ranges of the gradient B, drift, collision, and rotational transform frequencies for finite values of $e \phi / k T$.

In addition to the above work for bumpy tori, estimates of collislonal transport rates have also been made for the closely related concept of toroidally-linked minimum B mirrors. ${ }^{8-10}$ Such estimates have been based on a random walk analysis of particle orbit diffusion and 
generally do not consider the influence of the ambipolar field. These systems, as well as the bumpy torus, are characterized by localized regions in velocity and configuration space where orbits are not well confined and intersect the wall. Over certain ranges of collisonality, such regions can account for a large portion of the plasma transport.

In the present paper we examine neoclassical transport in a low beta bumpy torus for arbitrary values of the ambipolar field. The motivation behind such calculations arises from the fact that the large field limit is not generally valid close to the center of such devices and near the high beta electron annuli (necessary for macroscopic etability). In addition, the dependence of transport rates on the ambipolar field is of critical importance in the self-consistent determination of the field. We shall present transport coefficients obtained using the Landau collision operator; comparison of these with results using a number of model collision operators will be discussed in a separate publication.ll Only a radial component of the ambipolar field will be considered; recent heavy ion beam probe measurements ${ }^{12}$ on EBT in the stable $\mathrm{T}$-mode of operation have indicated the $\theta$ component of the ambipolar field is small relative to the radial component. Transport rates for a bumpy torus including both $E_{r}$ and $E_{\theta}$ ambipolar fleld componente were calculated in Ref. 7 using a Krook model collision operator. It was demonstrated there that in thc large field limite (eф/kT $\because 1)$ only the radial component $\mathrm{E}_{\mathrm{r}}$ is of importance in transport. The importance of the $E_{\theta}$ field when $e \phi / k T$ is finite has not been assessed yet and requires further research.

The transport cuelificients which are calculated in this work exhibit a novel, nonlinear dependence on the radial ambipolar field which does not occur, for example, in axisymmetric devices or simple gas discharges. Transport rates are sensitive to both the magnitude and direction of the ambipolar field - in the strong field limit, they scale as the inverse square of the electric field. For smaller field values, an even stronger dependence is obtained. The reasons for this behavior relate to the basic particle drift orbits and neoclassical step size. As in all toroidal devices, single particle confinement in a bumpy torus depends 
on compensation of the toroidal vertical drift by a poloidal rotation (caused in this case by bumpiness in the toroidal field and $\vec{E} \times \vec{B}$ drifts). Transport of particles and energy across field lines occurs when pitch angle and energy scattering randomly move particles onto orbits which are not as well confined as those prior to the scattering event. In the case of a bumpy torus, there are certain regions of velocity space (discussed in more detail in Sect. IV) where the poloidal drift becomes small and in which confinement is expected to be significantly degraded from the average. The size and direction of the ambipolar field directly controls the number of particles which are affected by such regions.

In addition to the dependence on the ambipolar electric field, bumpy torus transport coefficients are also a function of the ratio $(\nu / \Omega)$ of the plasma collisionality to the poloidal drift frequency. This dependence may be explained ${ }^{7}$ on the basis of heuristic arguments Involving single particle orbits. In the low collision frequency limit $(\nu / \Omega<1)$ where particles are able to trace out complete closed drift orbits before suffering a collision, the step size for transport scales as the horizontal displacement of the orbit away from the center of the bore $\left(\Delta \simeq v_{y} / \Omega\right.$, where $v_{y}=$ vertical drift due to toroidal curvature in the magnetic field). The diffusion coefficient thus scales as $D_{n} \simeq v\left(v_{y} / \Omega\right)^{2}$ where $\nu$ is the $90^{\circ}$ scattering frequency. However, in the high collision frequency limit $(\nu / \Omega \gg 1)$, where particles are not able to trace out closed drift orbits, the effective step size is reduced by the fraction of the nrhit which has been completed $\left[\Delta \simeq(\Omega / \nu)\left(v_{y} / \Omega\right)\right]$ on the avelaye before a scattering event occurs. In this case the diffusion coefficient, $D_{n} \simeq v_{y}^{2} / \nu$, scales inversely with respect to the plasma collisionality. In applying the results presented in this paper to specific experiments, it should be kept in mind that they are based on a number of approximations. The most important ones are mentioned here and elaborated on at appropriate polnts in the following analysis. (1) All trausport flows are one-dimensional (i.e., macroscopic quantities such as temperature and density have significant variations only in the radial direction). (2) The effective neoclassical step size is small relative to 
the scale lengths of gradients in plasma parameters (local approximation). (3) The lowest order part of the distribution function is a Maxwellian. (4) The $\theta$ dependence in the distribution function and particle orbits is treated by retaining only first order terms (proportional to $\sin \theta$ and $\cos \theta$ ) in a Fourier series. (5) The ambipolar field is purely radial. (6) Only classical Coulomb interactions are present in the collision term. (7) The specific results given here for transport coefficients have been evaluated near the central region of the plasma.

In Sect. II we derive a bounce-averaged drift kinetic equation. Next, the conflguration space dependence of the distribution function is treated by expanding the $\theta$ dependence and making a local approximatiun in radius. The transport coefficient matrix is given in terms of integrals over the Clssl vider distribution function. Affer this, the specific particle orbit model which has been used for the present results is discussed. Several numerical procedures for solving the kinetic equation are mentioned and results presented for transport coefficients. Finally, estimates are made of plasma particle and energy lifetimes based on the transport coefficients which have been obtained. 
II. BOUNCE-AVERAGED DRIFT KINETIC EQUATION

The motion of particles parallel to field lines in a bumpy torus is characterized either by a circulation around the torus or by trapping between adjacent mirrors. A consideration of the relative magnitudes of the collision and bounce frequencies reveals that there are typically very few collisions per bounce period.

$$
\frac{\nu}{\omega_{b}}=\frac{\nu R}{v_{t h}^{N}}=3.4 \times 10^{-4} \frac{(R / 150)\left(\mathrm{n} / 10^{12}\right)}{(\mathrm{N} / 24)(\mathrm{kT} / 100 \mathrm{eV})^{2}},
$$

where

$$
\begin{aligned}
& \mathrm{N}=\text { number of mirror sections, } \\
& \mathrm{n}=\text { plasma density }\left(\mathrm{cm}^{-3}\right), \\
& \mathrm{R}=\text { major radius of torus }(\mathrm{cm}), \text { and } \\
& \mathrm{T}=\text { plasma temperature }(\mathrm{eV}) .
\end{aligned}
$$

As may be seen from this formula, $v / \omega_{b} \ll 1$ for the present EBT device. A consideration of the parameters which are expected to characterize future, larger bumpy tori also indicates that such an ordering will rcmain true for these devices. This relation makes it desirable to average the rapid bounce motion since collisional transport occurs on a much slower time scale. Such an average also reduces the number of spatial dimensions which must be considered in the problem. Since experimental quantities are gencrally medsured only at the midplane, all bounce-averaged variables will be referred back to this point.

\section{A. Drift and Acceleration Terms}

Drift kinetic equations have been derived recently by a number of different authors.13,14 In obtaining a bounce-averaged drift kinetic equation for a bumpy torus it is convenient to use a bounce-averaged Liouville conservation relation in $\alpha, \beta, \mu, J$ phase space ${ }^{15}$ such as that given below. 


$$
\frac{\partial F}{\partial t}+\frac{\partial}{\partial \alpha}\left(\langle\dot{\alpha}>F)+\frac{\partial}{\partial \beta}\left(\langle\dot{\beta}>F)+\frac{\partial}{\partial J}(\dot{J} F)+\frac{\partial}{\partial \mu}(\dot{\mu} F)=\langle C F\rangle,\right.\right.
$$

where $\alpha$ and $\beta$ are field line coordinates with $\vec{B}=\vec{\nabla} \alpha \times \vec{\nabla} \beta$,

$$
\begin{aligned}
& \mu=\mathrm{mv}_{\perp}^{2} / 2 \mathrm{~B}, \\
& \varepsilon=\frac{1}{2} \mathrm{mv}^{2}+\mathrm{e} \phi, \\
& \mathrm{J}=\mathrm{m} \phi \mathrm{d} \ell \mathrm{v}_{\|}=\sqrt{2 \mathrm{~m}} \oint \mathrm{d} l \sqrt{\varepsilon-\mu \mathrm{B}-\mathrm{e} \phi}, \\
& \mathrm{F}(\alpha, \beta, \mu, \mathrm{J})=\langle\mathrm{f}\rangle=\tau^{-1} \oint \mathrm{d} \ell \mathrm{f} / \mathrm{v}_{\|}, \\
& \tau=\oint \mathrm{d} \ell / \mathrm{v}_{\|}, \\
& \mathrm{d} \ell=\text { differential length along a fleld line, } \\
& \phi=\text { electrostatic potential, } \\
& \text { brackets }<>\text { denote bounce averages, and } \\
&\langle\mathrm{C} f\rangle=\text { bounce-averaged collision operator. }
\end{aligned}
$$

The limits on field line integrals in these averages run frow the midplane to the turning point for mirror trapped particles and from the midplane to the mirror throat for circular1ng particles. The volume element in $J, \mu, \alpha, \beta, l$ space is related in the following way to the usual phase space volume element.

$$
\frac{4 \pi}{\mathrm{m}^{2} \mathrm{v}_{\|} \tau} \mathrm{d} \ell \mathrm{dJd} \mu \mathrm{d} \alpha \mathrm{d} \beta=4 \pi \mathrm{v}_{1} d \mathrm{v}_{1} \mathrm{dv}_{\|} \mathrm{d}^{3} \mathrm{x}
$$

The additional factor of two in Lq. (3) results from aceuming that $f\left(-v_{\|}\right)=f\left(v_{\|}\right)$so that only positive values of $v_{\|}$need be considered. Note that:

$$
\int_{\ell=0}^{\ell=\mathrm{L}} \frac{4 \pi}{\mathrm{m}^{2} \mathrm{v}_{\|} \tau} \mathrm{d} \ell \mathrm{dJ} \mathrm{d} \mu \mathrm{d} \alpha \mathrm{d} \beta \mathrm{f}=\frac{4 \pi}{\mathrm{m}^{2}} \mathrm{dJd} \mu \mathrm{d} \alpha \mathrm{d} \beta \mathrm{F} .
$$


Since the Jacobian involved in going from $v_{\perp}, v_{\|}$, and $\vec{x}$ to $J, \mu, \alpha, \beta$ is a constant, it is clear that integrating Eq. (1) over phase space results in a continuity or particle balance relation:

$$
\frac{\partial N}{\partial t}+\text { flux leaving volume }=\text { sources }
$$

Since $\mu$ and $J$ are conserved quantities in the bumpy torus, $\dot{\mu}=\dot{J}=0$. Also, the drift velocities $\langle\dot{\alpha}\rangle$ and $\langle\dot{\beta}\rangle$ may be related ${ }^{16}$ to the Hamiltonian $\mathrm{K}(\alpha, \beta, \mu, \mathrm{J})$ as indicated below.

$$
\begin{aligned}
& \langle\dot{\alpha}\rangle=-\frac{c}{\mathrm{e}} \frac{\partial \mathrm{K}}{\partial \beta} . \\
& \langle\dot{\beta}\rangle=\frac{\mathrm{c}}{\mathrm{e}} \frac{\partial \mathrm{K}}{\partial \alpha} .
\end{aligned}
$$

Using these relations, and transforming from $F(J, \mu, \alpha, \beta)$ to $\hat{F}(\varepsilon, \mu, \alpha, \beta)$, Eq. (1) may be reduced to the following simple form:

$$
\frac{\partial \hat{F}}{\partial t}+\langle\dot{\alpha}\rangle \frac{\partial \hat{F}}{\partial \alpha}+\langle\dot{\beta}\rangle \frac{\partial \hat{F}}{\partial \beta}=\langle\hat{C F}\rangle
$$

In the calculations that folluw, it is desirable to express Eq. (7) in a $v, x, r, \theta$ coordinate system [where $x$ is the pitch angle $=\cos ^{-1}\left(v_{\|} / v\right)$ ] The relations between $v, \chi$ and $\varepsilon, \mu$ are given below.

$$
\mathrm{v}=\sqrt{\frac{2}{\mathrm{~m}}(\varepsilon-\mathrm{e} \phi)} ; \quad \chi=\cos ^{-1}\left(1-\frac{\mu \mathrm{B}}{\varepsilon-\mathrm{e} \phi}\right)^{1 / 2}
$$

The transformation from $\alpha$ and $\beta$ to $r$ and $\theta$ is facil.itated by the following: 16 


$$
\begin{aligned}
& \left\langle\overrightarrow{\mathrm{v}}_{\mathrm{D}}\right\rangle=\frac{\mathrm{c}}{\mathrm{e} \dot{\mathrm{BT}}} \vec{\nabla} \mathrm{J} \times \hat{\mathrm{n}}, \\
& \langle\dot{\alpha}\rangle=\frac{\mathrm{c}}{\mathrm{e} \tau} \frac{\partial \mathrm{J}}{\partial \beta},
\end{aligned}
$$

and

$$
\langle\dot{\beta}\rangle=\frac{\mathrm{c}}{\mathrm{e} \tau} \frac{\partial \mathrm{J}}{\partial \alpha}
$$

Making the transformation from $\hat{F}(\varepsilon, \mu, \alpha, \beta)$ to $\bar{F}(v, x, r, \theta)$, Eq. (7) becomes:

$$
\begin{aligned}
\frac{\partial \bar{F}}{\partial t}+\left\langle\vec{v}_{D}\right\rangle \cdot \vec{\nabla} F+\frac{1}{2}\left\langle\vec{v}_{D}\right\rangle & \cdot\left(\vec{\nabla}_{\ln } \ln \right) \tan \chi \frac{\partial \bar{F}}{\partial x} \\
& +\frac{e}{m v}\left\langle\vec{v}_{D}\right\rangle \cdot \vec{E}\left(\frac{\partial \bar{F}}{\partial v}-\frac{\tan x}{v} \frac{\partial \bar{F}}{\partial x}\right)=\langle C \bar{F}\rangle .
\end{aligned}
$$

The kinetic equation given above has been written in a form particularly suited to the drift orbit model which will be described in Sect. IV. There the $r$ and $\theta$ components of $\left\langle\vec{v}_{D}\right\rangle$ will be specified based on bounce averages over a bumpy cylindcr model for the vacuum fielda. We note, hnwever, that for purposes of more accurate toroldal field and orbit models, it is not necessary to decompose $\left\langle\vec{v}_{D}\right\rangle$ into these components and Eq. (10) may be written directly in terms of the $J$ invariant, using relation $(y)$.

$$
\begin{aligned}
\frac{\partial \bar{F}}{\partial t}+\frac{c}{e B \tau r}\left\lceil\frac{\partial \bar{F}}{\partial r} \frac{\partial J}{\partial \theta}-\frac{\partial F}{\partial \theta} \frac{\partial J}{\partial r}+\frac{\tan \chi}{2} \frac{\partial \bar{F}}{\partial \chi} \times\left(\frac{\partial \bar{F}}{\partial r} \frac{\partial \ln B}{\partial \theta}-\frac{\partial F}{\partial \theta} \frac{\partial \ln B}{\partial r}\right)\right. \\
\left.+\frac{e}{m v}\left(E_{r} \frac{\partial J}{\partial \theta}-r E_{\theta} \frac{\partial J}{\partial r}\right)\left(\frac{\partial \bar{F}}{\partial v}-\frac{\tan X}{v} \frac{\partial \bar{F}}{\partial \chi}\right)\right]=\langle C \bar{F}\rangle .
\end{aligned}
$$

Such a form would be useful, for example, in directly utilizing calculations of $J$ and $\tau$ from tensor pressure, finite $B$, and $3-D$ equilibrium codes. ${ }^{2}$ 
B. Collision Operator

The collision operator for Coulomb interactions most generally used in plasma kinetic theory is the Fokker-Planck operator. This has been given ${ }^{1-19}$ for species $a$ as the following.

$$
\Gamma_{a}^{-1} \mathrm{Cf}_{a}=-\frac{\partial}{\partial v_{i}}\left(f_{a} \frac{\partial h_{a}}{\partial v_{i}}\right)+\frac{1}{2} \frac{\partial^{2}}{\partial v_{i} \partial v_{j}}\left(f_{a} \frac{\partial^{2} g_{a}}{\partial v_{i} \partial v_{j}}\right),
$$

where

$$
\begin{aligned}
\Gamma_{a} & =\frac{4 \pi z_{a}^{4} e^{4}}{m_{a}^{2}} \ln \Lambda_{a}, \\
\Lambda_{a} & =\frac{3 k T a}{z_{a}^{2} e^{2}}\left(\frac{k T_{a}}{4 \pi N_{e} e^{2}}\right)^{1 / 2}, \\
h_{a}(\vec{v}) & =\sum_{b}\left(1+\frac{m_{a}}{m_{b}}\right)\left(\frac{z_{b}}{z_{a}}\right)^{2} \int d^{3} v^{\prime} \frac{f_{b^{\prime}}\left(\vec{v}^{\prime}\right)}{\left|\vec{v}-\vec{v}^{\prime}\right|}, \\
g_{a}(\vec{v}) & =\sum_{b}\left(\frac{z_{b}}{z_{a}}\right)^{2} \int d^{3} v^{\prime} f_{b}\left(\vec{v}^{\prime}\right)\left|\vec{v}-\vec{v}^{\prime}\right|,
\end{aligned}
$$

and

$$
\text { 7. } a, z_{b}=\text { sharge numbers of speicles } a \text { and } b \text {. }
$$

The potentials $h_{a}$ and $g_{a}$ further satisfy the relations 17,19 given below. 


$$
\begin{aligned}
& \nabla^{2} h_{a}=-4 \pi \sum_{b}\left(1+\frac{m_{a}}{m_{b}}\right)\left(\frac{z_{b}}{z_{a}}\right)^{2} f_{b}(\vec{v}) . \\
& \nabla^{2} g_{a}=2 \sum_{b}\left(\frac{z_{b}}{z_{a}}\right)^{2} \int \frac{d^{3} v^{\prime} f_{b^{\prime}}\left(\vec{v}^{\prime}\right)}{\left|\vec{v}-\vec{v}^{\prime}\right|} . \\
& \nabla^{4} g_{a}=-8 \pi \sum_{b} f_{b}\left(\frac{z_{b}}{z_{a}}\right)^{2} .
\end{aligned}
$$

Using the above relations, Eq. (11) may be expanded into the followluy form:

$$
\mathrm{l}_{a}^{-1} \mathrm{Cf} a=\frac{\partial \mathrm{H}_{a}}{\partial v_{j}} \frac{\partial f_{a}}{\partial v_{j}}+\frac{1}{2} \frac{\partial^{2} g_{a}}{\partial v_{j} \partial v_{i}} \frac{\partial^{2} f_{a}}{\partial v_{j} \partial v_{i}}+4 \pi f_{a} \sum_{b} \frac{m_{a}}{m_{b}}\left(\frac{z_{b}}{Z_{a}}\right)^{2} f_{b},
$$

where

$$
H_{a}=\sum_{b}\left(\frac{z_{b}}{z_{a}}\right)^{2}\left(1-\frac{m_{a}}{m_{b}}\right) \int \frac{d^{3} v^{\prime} f_{b}\left(v^{\prime}\right)}{\left|\vec{v}-\vec{v}^{\prime}\right|} .
$$

This collision operator is bilinear in $\mathrm{f}_{\mathrm{a}}$ and it will be necessary to linearize it for the present application. This is achieved by breaking $f_{a}$ into a zero order, equilibrium part ( $f_{00}-$ usually taken as the Maxwellian) and a perturbed part $\left(f_{a l}\right)$; the resulting collision operator is then of the form given below.

$$
\mathrm{Cf}_{\mathrm{a}}=\mathrm{C}_{0} \mathrm{f}_{\mathrm{al}}+\mathrm{C}_{1} \mathrm{f}_{\mathrm{a} 0} \cdot
$$

The $C_{0} f_{a l}$ part involves velocity space derivatives of the unknown first order distribution, $f_{a l}$, while the $\mathrm{C}_{1} \mathrm{f}_{\mathrm{a} 0}$ part involves integrals over $f_{a l}$. In a v, $\chi$ velocity space coordinate system, the $C_{0} f_{a l}$ part of the 
collision term may be written as follows, using the relations given in Eq. (12).

$$
\begin{aligned}
& \Gamma_{\mathrm{a}}^{-1} \mathrm{C}_{0} \mathrm{f}_{\mathrm{al}}=\frac{1}{2} \frac{\partial^{2} \mathrm{~g}_{\mathrm{a} 0}}{\partial \mathrm{v}^{2}} \frac{\partial^{2} \mathrm{f}_{\mathrm{al}}}{\partial \mathrm{v}^{2}}+\left(\frac{\partial \mathrm{H}_{\mathrm{a} 0}}{\partial \mathrm{v}}+\frac{1}{\mathrm{v}^{2}} \frac{\partial \mathrm{g}_{\mathrm{a} 0}}{\partial \mathrm{v}}\right) \frac{\partial \mathrm{f}_{\mathrm{al}}}{\partial \mathrm{v}} \\
& +\frac{1}{2 \mathrm{v}^{3} \sin x} \frac{\partial}{\partial \chi}\left(\sin \times \frac{\partial \mathrm{f}_{\mathrm{al}}}{\partial \chi}\right) \frac{\partial \mathrm{g}_{\mathrm{a} 0}}{\partial \mathrm{v}}+4 \pi \mathrm{f}_{\mathrm{al}} \sum_{\mathrm{b}}\left(\frac{\mathrm{z}_{\mathrm{b}}}{\mathrm{z}_{\mathrm{a}}}\right)^{2} \frac{\mathrm{m}_{\mathrm{a}}}{\mathrm{m}_{\mathrm{b}}} \mathrm{f}_{\mathrm{b} 0},
\end{aligned}
$$

where

$$
g_{a 0}=\sum_{b}\left(\frac{z_{b}}{z_{a}}\right)^{2} \int d^{3} v^{\prime} f_{b 0}\left(v^{\prime}\right)\left|\vec{v}-\vec{v}^{\prime}\right|,
$$

and

$$
\mathrm{H}_{\mathrm{a} 0}=\sum_{\mathrm{b}}\left(\frac{\mathrm{z}_{\mathrm{b}}}{\mathrm{z}_{\mathrm{a}}}\right)^{2}\left(1-\frac{\mathrm{m}_{\mathrm{a}}}{\mathrm{m}_{\mathrm{b}}}\right) \int \frac{\mathrm{d}^{3} \mathrm{v}^{\prime} \mathrm{f}_{\mathrm{b}_{0}}\left(\mathrm{v}^{\prime}\right)}{\left|\overrightarrow{\mathrm{v}}-\overrightarrow{\mathrm{v}}^{\prime}\right|} .
$$

As a result of the symmetry of the collision operator [see Eq. (13)], the $C_{1} f_{a 0}$ part of the collision term is as given below.

$$
\begin{aligned}
\Gamma_{a}^{-1} \mathrm{C}_{1} \mathrm{f}_{\mathrm{a} 0} & =\frac{1}{2} \frac{\partial^{2} \mathrm{~g}_{\mathrm{al}}}{\partial \mathrm{v}^{2}} \frac{\partial^{2} \mathrm{f}_{\mathrm{a} 0}}{\partial \mathrm{v}^{2}}+\left(\frac{\partial \mathrm{H}_{\mathrm{al}}}{\partial \mathrm{v}}+\frac{1}{\mathrm{v}^{2}} \frac{\partial \mathrm{g}_{\mathrm{al}}}{\partial \mathrm{v}}\right) \frac{\partial \mathrm{f}_{\mathrm{a} 0}}{\partial \mathrm{v}} \\
& +\frac{1}{2 \mathrm{v}^{3} \sin \times} \frac{\partial}{\partial x}\left(\sin \times \frac{\partial \mathrm{g}_{\mathrm{al}}}{\partial \mathrm{x}}\right) \frac{\partial \mathrm{f}_{\mathrm{a} 0}}{\partial \mathrm{v}}+4 \pi \mathrm{f}_{\mathrm{a} 0} \sum_{\mathrm{b}}\left(\frac{\mathrm{z}_{\mathrm{b}}}{\mathrm{z}_{\mathrm{a}}}\right)^{2} \frac{\mathrm{m}_{\mathrm{a}}}{\mathrm{m}_{\mathrm{b}}} \mathrm{f}_{\mathrm{bl}}(\overrightarrow{\mathrm{v}}),
\end{aligned}
$$


where $H_{a l}$ and $g_{a l}$ are defined similarly to $H_{a 0}$ and $g_{a 0}$ except that $f_{b 0}$ is replaced by $f_{b l}$. The functions $g_{a 0}$ and $H_{a 0}$ may be expressed as ${ }^{17-19}$

$$
\begin{aligned}
g_{a 0}=4 \pi \sum_{b}\left(\frac{z_{b}}{z_{a}}\right)^{2}\left\{\int_{0}^{v} d v^{\prime} f_{b 0}\left(v^{\prime}\right) v v^{\prime 2}\left[1+\frac{1}{3}\left(\frac{v^{\prime}}{v}\right)^{2}\right]\right. \\
\left.+\int_{v}^{\infty} d v^{\prime} f_{b 0}\left(v^{\prime}\right) v^{\prime 3}\left[1+\frac{1}{3}\left(\frac{v^{\prime}}{v}\right)^{2}\right]\right\} ;(18) \\
H_{a 0}-4 \pi \sum_{b}\left(\frac{z_{b}}{\bar{z}_{a}}\right)^{2}\left(1-\frac{m_{a}}{m_{b}}\right)\left[\int_{0}^{v} \frac{v^{\prime 2}}{v} f_{b u^{\prime}}\left(v^{\prime}\right) d v^{\prime}+\int_{v}^{w} v^{\prime} f_{b 0}\left(v^{\prime}\right) d v^{\prime}\right] .
\end{aligned}
$$

Performing the gyroangle integrations ${ }^{1 \%, 1 y}$ in $\mathrm{g}_{\mathrm{al}}$ and $\mathrm{H}_{\mathrm{a}_{1}}$ (and leaving $f_{b l}$ an arbitrary function of $v$ and $x$ ) results in the formulas given below.

$$
\begin{aligned}
& g_{a l}=4 \sum_{b}\left(\frac{z_{b}}{z_{a}}\right)^{2} \int_{0}^{w} v^{\prime 2} d v^{\prime} \int_{0}^{\pi} \operatorname{cin} x^{\prime} d x^{\prime} f_{b l}\left(v^{\prime}, x^{\prime}\right) q E(p / q) \cdot(20) \\
& H_{a l}=4 \sum_{b}\left(\frac{z_{b}}{z_{a}}\right)^{2}\left(1-\frac{m_{a}}{m_{b}}\right) \int_{0}^{\infty} v^{\prime 2} d^{\prime} \int_{0}^{\pi} \sin x^{\prime} d x^{\prime} f_{b l}\left(v^{\prime}, x^{\prime}\right) \frac{k(p / q)}{q},
\end{aligned}
$$

where

$$
\begin{aligned}
E, K & =\text { elliptic integrals, } \\
p^{\prime} & =\sqrt{4 v v^{\prime} \sin \chi \sin \chi^{\prime}}, \text { and } \\
q & =\left[v^{2}+v^{\prime 2}-2 v v^{\prime}\left(\cos x \cos x^{\prime}-\sin x \sin \chi^{\prime}\right)\right]^{1 / 2} .
\end{aligned}
$$


In order to bounce average the collision operators given in Eqs. (15) and (17), it is first desirable to transform to a coordinate system with conserved independent variables such as $v$. and $\mu$ ( $\mu=$ magnetic moment) which do not depend on the particle's spatial position along its bounce orbit, i.e., let $f(v, X)=F(v, \mu)$. Then

$$
\frac{\partial f}{\partial v}=\frac{\partial F}{\partial v}+\frac{\partial F}{\partial \mu} \frac{\partial \mu}{\partial v}=\frac{\partial F}{\partial v}+\frac{2 \mu}{v} \frac{\partial F}{\partial \mu}
$$

where

$$
\mu=\frac{m v^{2}}{2 B} \sin ^{2} x
$$

and

$$
\frac{\partial f}{\partial \chi}=\frac{\partial F}{\partial \mu} \frac{\partial \mu}{\partial x}=\frac{m^{2}}{B} \sin x \cos x \frac{\partial F}{\partial \mu}
$$

Since $\partial f / \partial v$ does not depend on spatially varying quantities (except through F), the first and second terms of the operator given in Eq. (15) are bounce averaged simply by replacing $\mathrm{f}_{\mathrm{al}}$ by $\left\langle\mathrm{f}_{\mathrm{al}}\right\rangle$ where $\left\langle\mathrm{f}_{\mathrm{al}}\right\rangle=$ $\tau^{-1} \oint \mathrm{dlf}{ }_{\text {al }} / \mathrm{v}_{\|}$and $\tau=\oint \mathrm{d} \ell / \mathrm{v}_{\|}$. This is also all that needs to be done in bounce averaging the last term in Eq. (15). However, the pitch-angle derivative term does depend on spatial position and must be averaged as follows:

$$
\begin{aligned}
& \langle Q\rangle=\left\langle\frac{1}{2 v^{3} \sin \gamma \cdot \partial \gamma} \frac{\partial}{\partial x}\left(\sin x \frac{\partial f_{a l}}{\partial x}\right)\right\rangle=\left\langle\frac{m_{\|}}{v^{3}} \frac{\partial}{\partial \mu}\left(\frac{\mu v_{\|}}{B} \frac{\partial f_{a l}}{\partial \mu}\right)\right\rangle \\
& =\frac{\mathrm{m}}{\tau \mathrm{v}^{3}} \frac{\partial}{\partial \mu}\left(\mu \frac{\partial<\mathrm{f} \mathrm{al}^{>}}{\partial \mu} \oint \frac{\mathrm{d} \ell}{\mathrm{B}} \mathrm{v}_{\|}\right) \text {. }
\end{aligned}
$$

We now convert back to the $\mathrm{v}, \mathrm{X}_{\mathrm{m}}$ coordinate system where $\chi_{\mathrm{mn}}$ is understood to be the pitch angle at the midplane. 


$$
\cos x_{\mathrm{m}}=\left.\frac{\mathrm{v}_{\|}}{\mathrm{v}}\right|_{\text {midplane }}=\sqrt{\frac{\mathrm{mv}^{2}-2 \mu \mathrm{B}_{\mathrm{M}}}{\mathrm{mv}^{2}}},
$$

where $B_{M}=$ magnetic field at the midplane. In this system,

$$
\langle Q\rangle=\frac{B_{M}}{2 \tau v^{5} \sin x_{m} \cos x_{m}} \frac{\partial}{\partial x_{m}}\left(\tan x_{m} \frac{\partial<f_{a l}>}{\partial x_{m}} \oint \frac{d l}{B} v_{\| l}\right) .
$$

To summarize, the bounce average of Eq. (15) is then given by the following.

$$
\begin{gathered}
\Gamma_{a}^{-l}<C_{a 0} f_{a l}>=\frac{1}{2} \frac{\partial^{2} g_{a u}}{\partial v^{2}} \frac{\partial^{2}<f_{a 1}>}{\partial v^{2}}+\left(\frac{\partial H_{a 0}}{\partial v}+\frac{1}{v^{2}} \frac{\partial g_{a 0}}{\partial v}\right) \frac{\partial<f_{a l}>}{\partial v} \\
+\langle Q\rangle+4 \pi<f_{a 1}>\sum_{b}\left(\frac{z_{b}}{z_{a}}\right)^{2} \frac{m_{a}}{m_{b}} f_{b 0}
\end{gathered}
$$

Bounce averaging the integral part of the collision operator, as given in Eq. (17), proceeds in much the same way as the bounce averaging of Eq. (15) with the result given below.

$$
\begin{aligned}
& \Gamma_{a}^{-1}\left\langle\mathrm{C}_{a 1} f_{a 0}\right\rangle=\frac{1}{2} \frac{\partial^{2} f_{a 0}}{\partial v^{2}}\left\langle\frac{\partial^{2} g_{a l}}{\partial v^{2}}\right\rangle+\frac{\partial f_{a 0}}{\partial v}\left(\left\langle\frac{\partial \mathrm{H}_{a l}}{\partial v}\right\rangle+\frac{1}{v^{2}}\left\langle\frac{\partial g_{a l}}{\partial v}\right\rangle\right) \\
& +4 \pi f_{a 0} \sum_{b}\left(\frac{z_{b}}{z_{a}}\right)^{2} \frac{m_{a}}{m_{b}}\left\langle f_{b l}>\right. \\
& +\frac{B_{M}}{2 \tau v^{5} \sin \chi_{1 u} \cos \chi_{m}} \quad \frac{\partial f_{a 0}}{\partial v} \frac{\partial}{\partial \chi_{m}}\left(\tan \chi_{m} \Phi \frac{d \ell}{B} v_{\|} \frac{\partial g_{a l}}{\partial x}\right) .
\end{aligned}
$$


In order to complete the bounce averaging of this term, the quantities $\left\langle\partial^{2} g_{a l} / \partial v^{2}\right\rangle,\left\langle\partial H_{a l} / \partial v\right\rangle,\left\langle\partial g_{a l} / \partial v\right\rangle$, and $\phi(d \ell / B) v_{\|}\left(\partial g_{a l} / \partial \chi\right)$ must be calculated. These are obtained using Eqs. (20) and (21) for $\mathrm{g}_{\mathrm{al}}$ and $\mathrm{H}_{\mathrm{al}}$ and will not be written out in detail at this point. There are several possible methods to evaluate these derivatives of $\mathrm{g}_{\mathrm{al}}$ and $\mathrm{H}_{\mathrm{a} . \mathrm{l}}$. One is to use Eqs. (20) and (21) and calculate the derivatives either analytically or numerically. Unfortunately, bounce averages of these terms then become quite unwieldly, and if the derivatives are done numerically, large amounts of storage may be required. ${ }^{19}$ An alternate approach, leading to expressions which would be easier to bounce average than Eqs. (20) and (21) is to expand the pitch-angle dependence of $f_{a l}$ in a Legendre series and then calculate $\mathrm{g}_{\mathrm{al}_{1}}$ and $\mathrm{H}_{\mathrm{al}} \cdot \mathrm{H}_{\mathrm{al}}$ and $\mathrm{g}_{\mathrm{al}_{1}}$ are then expressed as an expansion in Legendre polynomials and may be readily bounce averaged. The necessary expressions to evaluate $g_{a l}$ and $\mathrm{H}_{\mathrm{al}}$ in this manner were indicated in Ref. 17. Such an approach has been used successfully, for example in Ref. 20; however, bounce averages were not performed in this work.

These results are arbitrary with respect to species. For the particular case treated in this paper (ion transport) the collision operator may be simplified somewhat. Due to the large value of the ionto-electron mass ratio, and since like-particle collisions can cause particle transport in nonaxisymmetric devices, it is adequate to retain only the ion-ion collision term and neglect the electron-ion collisions in solving the ion transport problem. This approximation results in $\mathrm{H}_{\mathrm{i} 0}=\mathrm{H}_{\mathrm{il}}=0$. In contrast, for electron transport calculations, it is necessary to consider both electron-electron and electron-ion collisions, as well as enhanced pitch-angle scattering by microwaves.21,22 In the following we shall be concerned only with ions; species subscripts will. be dropped for simplicity. 
THIS PAGE

\section{WAS INTENTIONALLY LEFT BLANK}


III. TREATMENT OF CONFIGURATION SPACE DEPENDENCE

The bounce-averaged kinetic Eq. (10) involves four dimensions: $r, \theta$ in configuration space and $v, \chi$ in velocity space. It is impractical, even with a numerical approach, to accurately treat any more than two of these at once. In the present analysis, the dimensionality is reduced by making approximations to the configuration space dependence in the distribution function and by treating the velocity space dependence more carefully. Other bumpy torus transport calculations are being pursued 22 where one dimension in velocity space $(v)$ and one dimension in configuration space $(r)$ are retained.

\section{A. Expansion of Theta Dependence}

A truncated Fourier series has been employed in treating the $\theta$ dependence (short way around the torus) in the first order part of the distribution as follows.

$$
f(r, \theta, v, \chi)=f_{o}(r, v)+f_{s}(r, v, \chi) \sin \theta+f_{c}(r, v, \chi) \cos \theta
$$

Such an approximation has also been utilized in earlier work, ${ }^{1,7}$ on bumpy torus transport coefficients and is motivated by a consideration of the single particle orbil characteristics. These orbits are, for the most part, circular in shape and shifted outward in major radius; they are thus amenable to the representation used in Eq. (28). Such an approximation neglects the fact that certail urblts w1ll deviate away from circular and may, in localized regions of velocity space, be banana shaped. As will be discussed in Sect. IV, such noncircular orbits are generally present when there is a cancellation between the poloidal $\vec{E} \times \vec{B}$ drifts and $\vec{\nabla} B \times \vec{B}$ drifts. Since the size of these regions in velocity space is relatively narrow, only a small fraction of the distribution is normally involved in such orbits, and they are not expected to significantly modify transport rates except possibly at very low collisionalities $(\nu / \Omega<1)$ where the transport due to scattering of the 
nearly circular orbits is small. We note that inclusion of such noncircular orbits would require retaining higher order terms in the $\theta$ expansion in Eq. (28) and would also necessitate the treatment of a $\theta$ dependence in the ambipolar potential.

The drift velocity $\left\langle\vec{v}_{D}\right\rangle$ may also be broken into components proportional to $1, \sin \theta$, and $\cos \theta$ as indicated below. The components of the drift velocity and coordinate system are shown in Fig. 1.

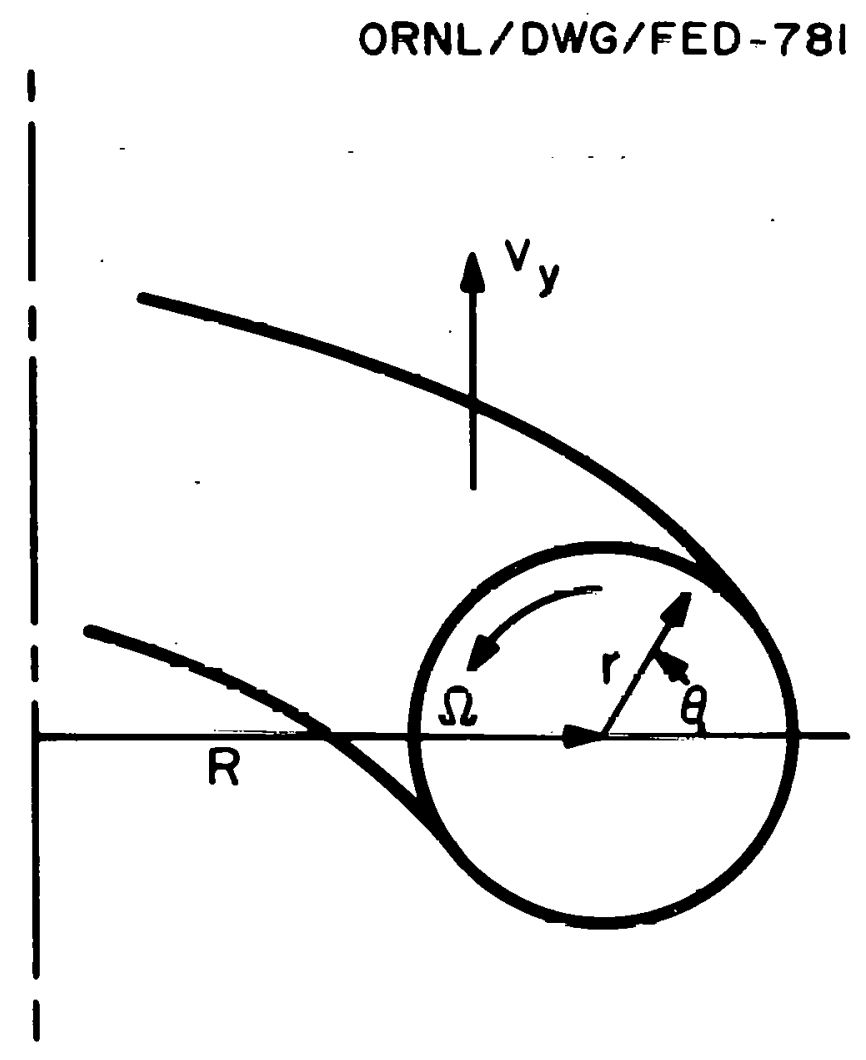

Fig. 1. Toroidal coordinate system and single particle drifts.

$$
\left\langle\overrightarrow{\mathrm{v}}_{\mathrm{D}}\right\rangle=\left\langle\mathrm{v}_{\mathrm{y}}\right\rangle \sin \theta \hat{\mathrm{e}}_{\mathrm{r}}+\left(\mathrm{r}\langle\Omega\rangle+\left\langle\mathrm{v}_{\mathrm{y}}\right\rangle \cos \theta\right) \hat{\mathrm{e}}_{\theta}
$$


where

$$
\begin{aligned}
& v_{y}=\text { vertical drift caused by the toroidal curvature } \\
&=\frac{v^{2}}{2 \omega_{c} R}\left(1+\frac{v_{\|}^{2}}{v^{2}}\right) \\
& \omega_{c}=\text { cyclotron frequency, } \\
& \Omega=\Omega_{E \times B}+\Omega_{\nabla B}, \\
& \Omega_{E \times B}=E_{r} / r B, \text { and } \\
& \Omega_{\nabla B}=\text { drift frequency in } \theta \text { direction due to the inhomogeneous } \\
& \text { magnetic field. }
\end{aligned}
$$

Substituting Eqs. (28) and (29) into the drift kinetic Eq. (10) and equating terms proportional to $\sin \theta$ and $\cos \theta$ separately to zero leads to the following set of coupled equations.

$$
\left\{\begin{array}{l}
\frac{\partial f_{s}}{\partial t}-\left\langle\Omega>f_{c}-\left\langle C_{o} f_{s}>-\left\langle C_{s} f_{o}>=-\left\langle v_{y}>\left(\frac{\partial f_{o}}{\partial r}+\frac{e E_{r}}{m v} \frac{\partial f_{o}}{\partial v}\right)\right.\right.\right.\right. \\
\frac{\partial f}{\partial t}+\left\langle\Omega>f_{s}-\left\langle C_{o} f_{c}>-\left\langle C_{c} f_{o}>=0 .\right.\right.\right.
\end{array}\right.
$$

In deriving these, we have assumed that $f_{s}$ and $f_{c}$ are of order $v_{y} / \Omega$ while terms of $O\left(v_{y}^{2} / \Omega^{2}\right)$ and higher have been neglected. Also, it is assumed that $\mathrm{f}_{\mathrm{o}}$ is a Maxwellian distribution function.

$$
f_{0}(r, v)=n(r)\left[\frac{m}{2 \pi k T(r)}\right]^{3 / 2} e^{-m v^{2} / 2 k T(r)} .
$$

Substituting this into Eq. (30), one obtains the following: 


$$
\begin{aligned}
\frac{\partial f_{s}}{\partial t}-\left\langle\Omega>f_{C}-\left\langle C_{o} f_{s}\right\rangle\right. & -\left\langle C_{s^{\prime}} f_{o}\right\rangle \\
& =-\left\langle v_{y}>\left[\frac{n^{\prime}}{n}+\left(\frac{E}{k T}-\frac{3}{2}\right) \frac{T^{\prime}}{T}+\frac{e \phi}{k T} \frac{\phi^{\prime}}{\phi}\right] f_{o},\right.
\end{aligned}
$$

where $\mathrm{E}=1 / 2 \mathrm{mv}^{2}$.

B. Local Approximation to Radial Dependence

A local approximation in the radial dependence is a necessary step in order to define neoclassical transport coefficients. The validity of this depends on the average step size characteristic of the transport process being small relative to gradient scale lengths in macroscopic variables; otherwise, a nonlocal transport theory is required. In Sect. V, estimates of the ratio of the effective step size to the plasma minor radius will be given based on the results presented there for transport coefficients. This parameter is useful in determining the range of validity of these results.

T.nrally in radius, density, temperature, and potential gradients appearing in the driving term on the right-hand side of Eq. (32) are independent quantities. If these gradients are not large relative to the inverse diffusion step size, then $f_{c}$ and $f_{s}$ distributions may be expanded including only first order derivatives in density, temperature, and potential.

$$
\begin{aligned}
& f_{s}=f_{s n} \frac{\mathrm{Il}^{\prime}}{\mathrm{n}}+\mathrm{f}_{s T} \frac{\mathrm{T}^{\prime}}{\mathrm{T}}+\mathrm{f}_{s e} \frac{\phi^{\prime}}{\phi} ; \\
& \mathrm{f}_{\mathrm{c}}=\mathrm{f}_{\mathrm{cn}} \frac{\mathrm{n}^{\prime}}{\mathrm{n}}+f_{c T} \frac{\mathrm{T}^{\prime}}{\mathrm{T}}+\mathrm{f}_{\mathrm{ce}} \frac{\phi^{\prime}}{\phi} .
\end{aligned}
$$

Substituting the above approximation into Eqs. (31) and (32) and setting coefficients of the density, temperature, and pocentral yradients independently to zero leads to the following three sets of coupled equations. 


$$
\begin{aligned}
& \left\{\begin{array}{l}
\frac{\partial f_{s n}}{\partial t}-\left\langle C_{o} f_{s n}>-<C_{s n} f_{o}>-<\Omega>f_{c n}=-<v_{y}>f_{0}\right. \\
\frac{\partial f_{c n}}{\partial t}-\left\langle C_{o} f_{c n}>-\left\langle C_{c n} f_{o}>+\left\langle\Omega>f_{s n}=0\right.\right.\right.
\end{array}\right. \\
& \left\{\begin{array}{l}
\frac{\partial f_{s T}}{\partial t}-\left\langle C_{o} f_{s T}>-\left\langle C_{s T} f_{o}>-\left\langle\Omega>f_{c T}=\left\langle v_{y}>\left(\frac{E}{k T}-\frac{3}{2}\right) f_{0}\right.\right.\right.\right. \\
\frac{\partial f_{c T}}{\partial t}-\left\langle C_{0} f_{c T}>-\left\langle C_{c T} f_{0}>+<\Omega>f_{s T}=0 .\right.\right.
\end{array}\right. \\
& \left\{\begin{array}{l}
\frac{\partial f_{s e}}{\partial t}-\left\langle C_{o} f_{s e}>-\left\langle C_{s e} f_{o}>-\left\langle\Omega>f_{c e}=-\frac{e \phi}{k T}\left\langle v_{y}>f_{o}\right.\right.\right.\right. \\
\frac{\partial f}{\partial t}-\left\langle C_{o} f_{c e}>-\left\langle C_{c e} f_{o}>+\left\langle\Omega>f_{s e}=0 .\right.\right.\right.
\end{array}\right.
\end{aligned}
$$

Methods for solving these coupled equations for the functions $f_{s n}$, $f_{c n}$, $f_{s T}$. . ., $f_{s e}, f_{c e}$ will be discussed in the next section. Since the driving term on the right-hand side of the coupled Eq. (36) is just eq/kT times that of Eq. (34), the solutions, $f_{s e}, f_{c e}$ of Eq. (36), are $e \phi / k T$ times those of Eq. (34), $\mathrm{f}_{\mathrm{sn}}, \mathrm{f}_{\mathrm{cn}}$.

\section{Expreesione for Traneport Cocfficiento}

The $\theta$-averaged radial particle and energy fluxes are given by integrating $\left\langle\mathrm{v}_{\mathrm{dr}}\right\rangle$ (radial component of the drift velocity) and $\left\langle\mathrm{v}_{\mathrm{dr}}\right\rangle$ times the kinetic energy over the distribution function. This form for the fluxes has been verified by Jaeger et $a 1 .{ }^{23}$ for the bumpy torus directly from the drift kinetic Eq. (10). 


$$
\begin{aligned}
& \text { Particle flux }=S_{r}=\frac{1}{2 \pi} \int_{0}^{2 \pi} \mathrm{d} \theta \int \mathrm{d}^{3} \mathrm{v}<\mathrm{v}_{\mathrm{dr}}>\mathrm{f} ; \\
& \text { energy } \mathrm{flux}=\mathrm{Q}_{\mathrm{r}}=\frac{1}{2 \pi} \int_{0}^{2 \pi} \mathrm{d} \theta \int \mathrm{d}^{3} \mathrm{v}\left(\frac{\mathrm{mv}}{2}\right)<\mathrm{v}_{\mathrm{dr}}>\mathrm{f},
\end{aligned}
$$

where $\left\langle v_{\mathrm{dr}}\right\rangle=\left\langle\mathrm{v}_{\mathrm{y}}\right\rangle \sin \theta$. Substituting Eqs. (28) and (33) into Eq. (37) results in the following.

$$
\begin{aligned}
& s_{r}-\frac{1}{2} \int d^{3} v v_{y}>\left(f_{s n} \frac{n^{\prime}}{n}+f_{s T} \frac{T^{\prime}}{T}+f_{s e} \frac{\phi^{\prime}}{\phi}\right) \\
& Q_{r}=\frac{1}{2} \int d^{3} v\left(\frac{m^{2}}{2}\right)<v_{y}>\left(f_{s n} \frac{n^{\prime}}{n}+f_{s T} \frac{T^{\prime}}{T}+f_{s e} \frac{\phi^{\prime}}{\phi}\right) .
\end{aligned}
$$

The six transport coefficients which relate particle and energy fluxes to gradients in temperature, density, and potential are normally defined as follows.

$$
\begin{aligned}
& S_{r}=-D_{n} \frac{d n}{d r}-D_{T} \frac{d T}{d r}-n \mu_{n} \frac{d \phi}{d r} . \\
& Q_{r}=-K_{n} \frac{d n}{d r}-K_{T} \frac{d T}{d r}-n \mu_{E} \frac{d \phi}{d r} .
\end{aligned}
$$

Comparison with Eqs. (38) and (39) then indicates that these transport coefficients may be related to the following moments of $f_{s n}$ and $f_{s T}$.

$$
\begin{aligned}
& \mathrm{D}_{\mathrm{n}}=-\frac{1}{2 \mathrm{n}} \int \mathrm{d}^{3} \mathrm{v}<\mathrm{v}_{\mathrm{y}}>\mathrm{f}_{\mathrm{sn}} . \\
& \mathrm{D}_{\mathrm{T}}=-\frac{1}{2 k \mathrm{~T}} \int \mathrm{d}^{3} \mathrm{v}<\mathrm{v}_{\mathrm{y}}>\mathrm{f}_{\mathrm{sT}} .
\end{aligned}
$$




$$
\begin{aligned}
& \mathrm{K}_{\mathrm{n}}=-\frac{1}{2 \mathrm{n}} \int \mathrm{d}^{3} \mathrm{v}\left(\frac{\mathrm{mv^{2 }}}{2}\right)<\mathrm{v}_{\mathrm{y}}>\mathrm{f}_{\mathrm{sn}} . \\
& \mathrm{K}_{\mathrm{T}}=-\frac{1}{2 \mathrm{kT}} \int \mathrm{d}^{3} \mathrm{v}\left(\frac{\mathrm{mv}}{2}\right)<\mathrm{v}_{\mathrm{y}}>\mathrm{f}_{\mathrm{sT}} .
\end{aligned}
$$

Since the solutions $f_{s e}$, $f_{c e}$ of Eq. (36) are simply e $\phi / k T$ times those of Eq. (34), the particle and energy mobilities, $\mu_{n}$ and $\mu_{E}$, are connected to $D_{n}$ and $K_{n}$ by Einstein relations.

$$
\mu_{\mathrm{n}}=\frac{\mathrm{e}}{\mathrm{kT}} \mathrm{D}_{\mathrm{n}} ; \quad \mu_{\mathrm{E}}=\frac{\mathrm{e}}{\mathrm{kT}} \mathrm{K}_{\mathrm{n}} \text {. }
$$




\section{THIS PAGE}

\section{WAS INTENTIONALLY \\ LEFT BLANK}




\section{PARTICLE ORBIT MODEL}

Guiding center particle orbits are a fundamental component in calculations of neoclassical transport rates. Not only is the average step size closely related to orbit characteristics, but so also is the fraction of velocity space in which the predominant transport losses occur. As a result, the effective collision frequency or degree of scattering required to take a particle from the well confined to the lossy region of velocity space is determined by orbit parameters.

For the results presented in this paper, a particular drift orbit model has been chosen which we describe in the present section. A basic assumption inherent in this model is that the aspect ratio of the torus is sufficiently large so that drifts introduced by the toroidal curvature represent, on the average, a small correction to the straight mirror field drifts and motion along field lines. Specifically, the particle drifts are broken into two components: (1) the rotation $(\Omega)$ around the $\theta$ direction caused by inhomogeneous mirror field drifts and $\vec{E} \times \vec{B}$ drifts (related to the radial ambipolar field); and (2) the toroidal vertical drift $\left(v_{y}\right)$ caused by toroidal curvature. It is assumed that the rotation frequency $\Omega$ may be calculated without consideration of the toroidal curvature, i.e., in a bumpy cylinder. Also, the bounce average of $v_{y}$ will be performed using bumpy cylinder vacuum magnetic fields.

\section{A. Bounce-Averaged Poloidal Drift Frequency}

As mentioned earlier, the poloidal drift frequency $\Omega$ is composed of the following two parts:

$$
\Omega_{1}=\Omega_{E \times B}+\Omega_{V B},
$$

where $\Omega_{E \times B}=-\vec{\nabla} \phi / r \vec{B}$, and $\Omega_{\nabla B}=$ drift due to inhomogeneous mirror fields. $\Omega_{\nabla B}$ is calculated using the longitudinal invariant $J$ and is thus a bounce-averaged quantity. Since the ambipolar potential may usually be approximated with a parabola, $\phi(r)=\phi_{0}\left(I-r^{2} / a^{2}\right)$, and $\phi_{0}$ is not expected to vary significantly along a field line (or bounce orbit), 
$\Omega_{\vec{E} \times \vec{B}}$ is effectively bounce averaged simply by specifying the $B$ field in the denominator to be the average seen by a particle in its bounce motion, $\left\langle B^{-l}\right\rangle=\tau^{-1} \oint \mathrm{d} l / v_{||}$. For the purposes of calculating $\Omega_{\nabla B}$, we shall consider the vacuum magnetic field produced in a straight cylinder carrying a surface current that is sinusoidally distributed in the $z$-direction. The magnetic fields interior to the cylinder are then given by: 24

$$
\begin{aligned}
& B_{r}=-B_{0} E_{l}(r) \sin k Z ; \\
& B_{Z}=B_{0}\left[1-E_{0}(r) \cos k Z\right],
\end{aligned}
$$

where

$$
\begin{aligned}
& k=2 \pi / d, \\
& \mathrm{~d}=\text { distance between mirrors, } \\
& E_{0}(r)=\left(\frac{M_{0}-1}{M_{0}+I}\right) I_{0}(k r) \text {, } \\
& E_{I}(r)=\left(\frac{M_{0}-1}{M_{0}+1}\right) I_{1}(k r) \text {, } \\
& B_{0}=\frac{M_{o}+1}{2 M_{0}} B_{\max }, \\
& M_{0}=\text { mirróor racto on axls, and } \\
& \text { - } \mathrm{I}_{\mathrm{o}}, \mathrm{I}_{1}=\operatorname{modified} \text { Bessel functions. }
\end{aligned}
$$

The longitudinal invariant $\mathrm{J}$ is given by:

$$
\mathrm{J}=\mathrm{m} \oint \mathrm{d} \ell \mathrm{v}_{\|}
$$

where the integration is understood to be along a field line over onehalf the field period (a distance d/2) for untrapped particles and over 
one-half of the particle's bounce period for trapped particles. Using Eq. $(9), \Omega_{\nabla B}$ is given by:

$$
\Omega_{\nabla B}=\frac{1}{m r \omega_{c} \tau} \frac{\partial J}{\partial r}
$$

where $\tau=\oint \mathrm{d} \ell / v_{\|}$is the period of the bounce motion. $\mathrm{d} \ell$ and $v_{\|}$are given by the following:

$$
\begin{aligned}
\mathrm{d} \ell & =\sqrt{1+(\mathrm{dr} / \mathrm{dz})^{2}} \mathrm{dz}=\sqrt{1+\left(\mathrm{B}_{\mathrm{r}} / \mathrm{B}_{\mathrm{Z}}\right)^{2}} \mathrm{dz} \\
& =\left(\mathrm{B} / \mathrm{B}_{\mathrm{Z}}\right) \mathrm{dz} \\
\mathrm{v}_{\|} & =\mathrm{v}_{\| \mathrm{m}} \sqrt{1-\mathrm{k}^{2} \sin ^{2} \mathrm{kZ} / 2},
\end{aligned}
$$

where $v_{\| m}=$ parallel velocity at the midplane, $v_{\perp m}=$ perpendicular velocity at the midplane; and

$$
\begin{aligned}
k^{2} & =\frac{z_{\perp}^{2}}{z_{\|}^{2}}[M(r)-1], \\
z_{\perp} & =v_{\perp m} / v, \\
z_{\|} & =v_{\| m} / v, \text { and } \\
M(r) & =\text { mirror ratio at radius } r .
\end{aligned}
$$

SuBstleuting Éqs. $(b 1)$ and ( $(22)$ into $J$ and $\tau$ results in the following.

$$
\begin{aligned}
& \mathrm{J}=\mathrm{mv}_{\| \mathrm{m}} \oint \mathrm{dz} \frac{\mathrm{B}}{\mathrm{B}_{\mathrm{Z}}} \sqrt{1-\mathrm{k}^{2} \sin ^{2} \mathrm{kZ} / 2} . \\
& \tau=\frac{1}{\mathrm{v}_{\| \mathrm{m}}} \oint \mathrm{dZ} \frac{\mathrm{B}}{\mathrm{B}_{\mathrm{Z}}} \frac{1}{\sqrt{1-\mathrm{k}^{2} \sin ^{2} \mathrm{kZ} / 2}} .
\end{aligned}
$$


For $\kappa^{2}>1$, particles are trapped between mirrors, while for $\kappa^{2}<1$, particles are untrapped. In the approximation that $\mathrm{B} / \mathrm{B}_{\mathrm{Z}}=1$, the above integrations may be carried out and lead to the results given below.

$$
\begin{aligned}
& \frac{\mathrm{J}}{\mathrm{m}}= \begin{cases}\frac{2 \mathrm{v}_{\| \mathrm{m}}}{\mathrm{k}} \kappa\left[\mathrm{E}\left(\frac{1}{\kappa^{2}}\right)-\left(1-\frac{1}{\kappa^{2}}\right) \mathrm{k}\left(\frac{1}{k^{2}}\right)\right] & \kappa^{2}>1 \\
2 \mathrm{v}_{\| \mathrm{m}} \mathrm{E}\left(\kappa^{2}\right) & \kappa^{2}<1 . \\
\cdots \mathrm{k} & \end{cases} \\
& \tau= \begin{cases}\frac{2}{k v_{\| m^{k}}} k\left(\frac{1}{k^{2}}\right) & k^{2}>1 \\
\frac{2}{k v_{\| m}} k\left(\kappa^{2}\right) & \kappa^{2}<1 .\end{cases} \\
& \mathrm{E}, \mathrm{K}=\text { complete elliptic integrals. }
\end{aligned}
$$

Particles with $k^{2}>1$ are locally trapped in the mirror sections while those with $\kappa^{2}<1$ are able to circulate around the torus. Using the relations,

$$
\frac{\partial}{\partial r} \ln v_{\| m}=\frac{1}{4} \frac{\kappa^{2}}{E_{j}(r)} \frac{\partial E_{o}}{\partial r},
$$

and

$$
\frac{\partial}{\partial r} \ln k^{2}=\frac{1}{E_{0}} \frac{\partial E_{0}}{\partial \mathrm{r}}\left(1-\frac{\kappa^{2}}{2}\right),
$$

one obtains the following result for $\Omega_{\nabla B}$.

$$
\Omega_{\nabla B}=\Omega_{0} \Omega^{\prime}
$$


where

$$
\Omega_{0}=\frac{2 \pi^{2}}{\omega_{c} d^{2}}\left(\frac{2 k T}{m}\right)\left[\frac{I_{l}(k r)}{k r I_{0}\left(k_{r}\right)}\right],
$$

and

$$
\Omega^{\prime}=c^{2} z_{\|}^{2} \begin{cases}\kappa^{2}\left[\frac{E\left(k^{-2}\right)}{k\left(\kappa^{-2}\right)}-\frac{1}{2}\right] & \kappa^{2}>1 \\ \frac{E\left(\kappa^{2}\right)}{k\left(k^{2}\right)}-1+\frac{\kappa^{2}}{2} & k^{2}<1 . \\ c^{2}=m v^{2} / 2 k T .\end{cases}
$$

As a result of the approximation made in Eqs. (56) and (57) that $\mathrm{B} / \mathrm{B}_{Z}=1$, the above expression is not accurate near $\mathrm{v}_{\|} / \mathrm{v}=1\left(\kappa^{2}=0\right)$. This situation is remedied by separately considering the special case, $\mathrm{v}_{\|} / \mathrm{v}=1$, and matching this solution to the one given above. At $v_{\|} / v=1$, $J$ and $\tau$ reduce to the integrals given below.

$$
\begin{aligned}
& \mathrm{J}=\mathrm{mv}_{\| \mathrm{m}} \oint \mathrm{d} \ell=\mathrm{mv}_{\| \mathrm{m}} \mathrm{L} / \mathrm{N} . \\
& \tau=\frac{1}{\mathrm{v}_{\| \mathrm{m}}} \oint \mathrm{d} \ell=\frac{\mathrm{L}}{\mathrm{Nv}_{\| \mathrm{m}}},
\end{aligned}
$$

where $\mathrm{L}=$ length of a field line, and $\mathrm{N}=$ number of mirrors. The precession frequency $\Omega_{\nabla B}$ is then given by:

$$
\Omega_{\nabla B}=\frac{v^{2}}{r \omega_{c} L} \frac{\partial L}{\partial r} .
$$


In order to evaluate $L$, the following approximation is used for $B / B_{Z}$.

$$
\frac{B}{B_{Z}}=\sqrt{1+\frac{E_{1}^{2} \sin k Z}{1-E_{0} \cos k Z}} \simeq 1+\frac{1}{2} \frac{E_{1}^{2} \sin ^{2} k Z}{1-E_{0} \cos k Z} .
$$

$\mathrm{L} / \mathrm{N}$ then may be calculated as indicated below:

$$
\frac{\mathrm{L}}{\mathrm{N}}=\oint \mathrm{dZ} \frac{\mathrm{B}}{\mathrm{B}_{\mathrm{Z}}}=\frac{\mathrm{d}}{2}+\frac{E_{1}^{\mathrm{Z}}}{2} \int_{0}^{\mathrm{d} / 2} \frac{\sin ^{2} \mathrm{kZdZ}}{\left(1-F_{0} \cos \mathrm{kZ}\right)^{2}} .
$$

Retaining terms of order $E_{1}^{2}$ and $E_{1}^{2} E_{0}^{2}$ the above becomes:

$$
\frac{\mathrm{L}}{\mathrm{N}}=\frac{\mathrm{d}}{2}\left[1+\frac{\tau_{\mathrm{I}}^{2}}{4}\left(1+\frac{3 E_{\mathrm{o}}^{2}}{4}\right)\right] \text {. }
$$

Typically $M_{U}=2$ and $E_{0}=1 / 3$ so that the final term above may be neglected. In this case $\Omega_{\nabla B}$ is given as:

$$
s \varepsilon_{\nabla B} \simeq \frac{v^{2}}{r \omega_{c}}\left(\frac{M_{o}-1}{M_{o}+I}\right)^{2} \frac{\partial I_{1}^{2}(k r)}{\partial r}
$$

Near the axis $r=0$, the above correction to $\Omega$ becomes:

$$
\dot{\Omega}_{\nabla \mathrm{R}} \simeq \frac{\mathrm{v}^{2} \mathrm{k}^{2}}{8 \omega_{\mathrm{c}}}\left(\frac{M_{0}-1}{M_{0}+1}\right)^{2}=\frac{c^{2} \Omega_{0}}{2}\left(\frac{M_{0}-1}{M_{0}+1}\right)^{2} .
$$


Thus

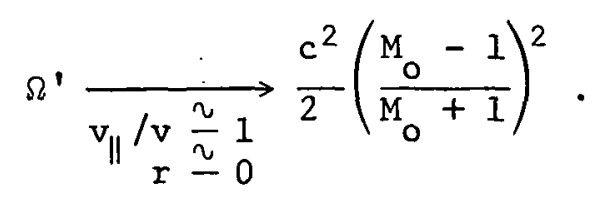

The result for $\Omega^{\prime}$ given in Eq. (60) may be modified to take into account the above correction by adding a linear function of the form given below.

$$
\frac{c^{2}}{2}\left(\frac{M_{0}-1}{M_{0}+1}\right)^{2}\left(\frac{v_{\|} / v-1 / \sqrt{2}}{1-1 / \sqrt{2}}\right) .
$$

This goes to the above result [Eq. (68)] when $v_{\|} / v=1$ and is zero at $v_{\|} / v=1 / 2\left(\kappa^{2}=1\right)$, where it may be shown that the correction term should vanish.

A plot of the $\Omega_{\nabla B}$ function is presented in Fig. 2 vs the velocity variables, $|\vec{v}| / v_{\text {th }}$ and $v_{\|} / v$ (= cos of pitch angle). As may be seen, $\Omega_{\nabla B}$ is largest for the high pitch-angle particles (which are locally trapped in mirror sections); this group will then have the best centered orbits. In the transition region between trapped and untrapped populations, $\Omega_{\nabla B}$ passes through zero twice and has a cusped region. This is related to trapped/circulating orbits which are just beginning to become circulating/ trapped and experience nearly equal amounts of positive and negative curvature. Such orbits will be shifted o!t near the wall and more exact calculations $^{2}$ indicate that some of these will be banana shaped. Since $\Omega_{\nabla B}$ in the present work is based on a bumpy cylinder model and thus has no $\theta$ dependence, the banana shaped orbits are not included here. We note that this approximation may lead to a slight overestimate of the transport losses since an orbit which is unconfined $\left(\Omega_{\nabla B}=0\right)$ in the bumpy cylinder model may actually turn out to be confined in the more exact toroidal calculation. The region in velocity space where $\Omega_{\nabla B}=0$ tends to be very lossy, and a significant portion of the particle and 
energy losses may occur near this region or as particles scatter into it. Significant losses can also be associated with the scattering of particles from locally trapped to circulating orbits (which are shifted farther off-center).

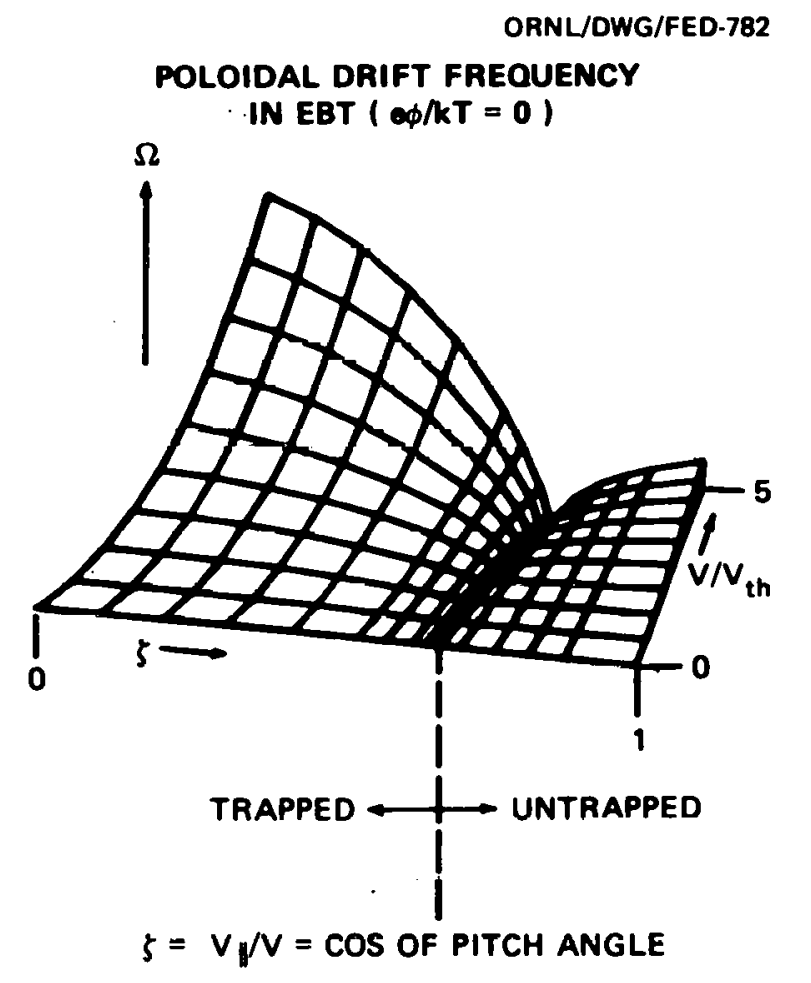

Fig. 2. Poloidal drift frequency due to curvature and gradients in a bumpy cylinder field $(\vec{E} \times \vec{B}$ drifts are not included) $\mathrm{vs} v / \mathrm{v}_{\text {th }}$ and $\zeta(=\cos \chi)$.

Since ambipolar fields are generally present, $\Omega_{\vec{E} \times \vec{B}}$ adds onto $\Omega_{\nabla B}$ and the total rotation frequency $\Omega$ will have the same shape as the function plotted in Fig. 2 but shifted up or down relative to the $\Omega=0$ plane (depending on the sign of $\mathrm{E}_{\mathrm{r}}$ and species). Agatn the reglons near $\Omega=0$ - where there is a cancellation between $\Omega_{\nabla B}$ and $\Omega_{\vec{E} \times \vec{B}}$ - will be particularly lossy and are of importance in particle and energy transport. The exact location of such regions is sensitive to the species, sign, and magnitude of the electric field. For ions and large positive 
electric fields, the loss region is moved out to high velocities where there are relatively few particles. In this case, transport is related mostly to the scattering of locally trapped particles into the circulating population. For ions and negative electric fields, the loss region is at lower velocities and can affect more of the distribution. Similar statements apply to electrons (except reversed). In Fig. 3 the location of the $\Omega=0$ region is plotted vs $v_{\|}$and $v_{\perp}$ for ions as a function of the ambipolar field for EBT-I parameters.

For the numerical calculations to be discussed in the following section, it is useful to have a simple analytic fit to the function $\Omega^{\prime} / \mathrm{c}^{2}$ vs $\mathrm{v}_{\| .} / \mathrm{v}$. In Fig. $4, \Omega^{\prime} / \mathrm{c}^{2}$ is plotted as given exactly by Eq. (60) plus the correction term of Eq. (69). Also plotted is a three-parameter fit which has been used extensively in the numerical calculations (particularly in the functional representation method). This fit is given below:

$$
\frac{\Omega^{\prime}}{c^{2}}=\frac{1}{2}-1.6\left(\frac{v_{\|}}{v}\right)^{2}+1.2\left(\frac{v_{\|}}{v}\right)^{4} .
$$

In Eq. (60), a normalization factor $\Omega_{u}$ was given for $\Omega_{\nabla B}$. Since the total precessional drift frequency $\Omega$ used in the following calculations will generally consist of a component from the $\vec{E} \times \vec{B}$ drifts as well, we define a normalization for the total drift frequency as given below.

$$
\Omega=\Omega_{0}\left(\alpha_{1} \Omega_{\nabla B}^{\prime}+\alpha_{2} \Omega_{\vec{E} \times \vec{B}}^{\prime}\right)
$$

Here

$$
\Omega_{0}=\frac{N^{2} k T}{2 e B R^{2}}\left[1+\frac{2 e E_{r}}{r k T}\left(\frac{R}{N}\right)^{2}\right],
$$



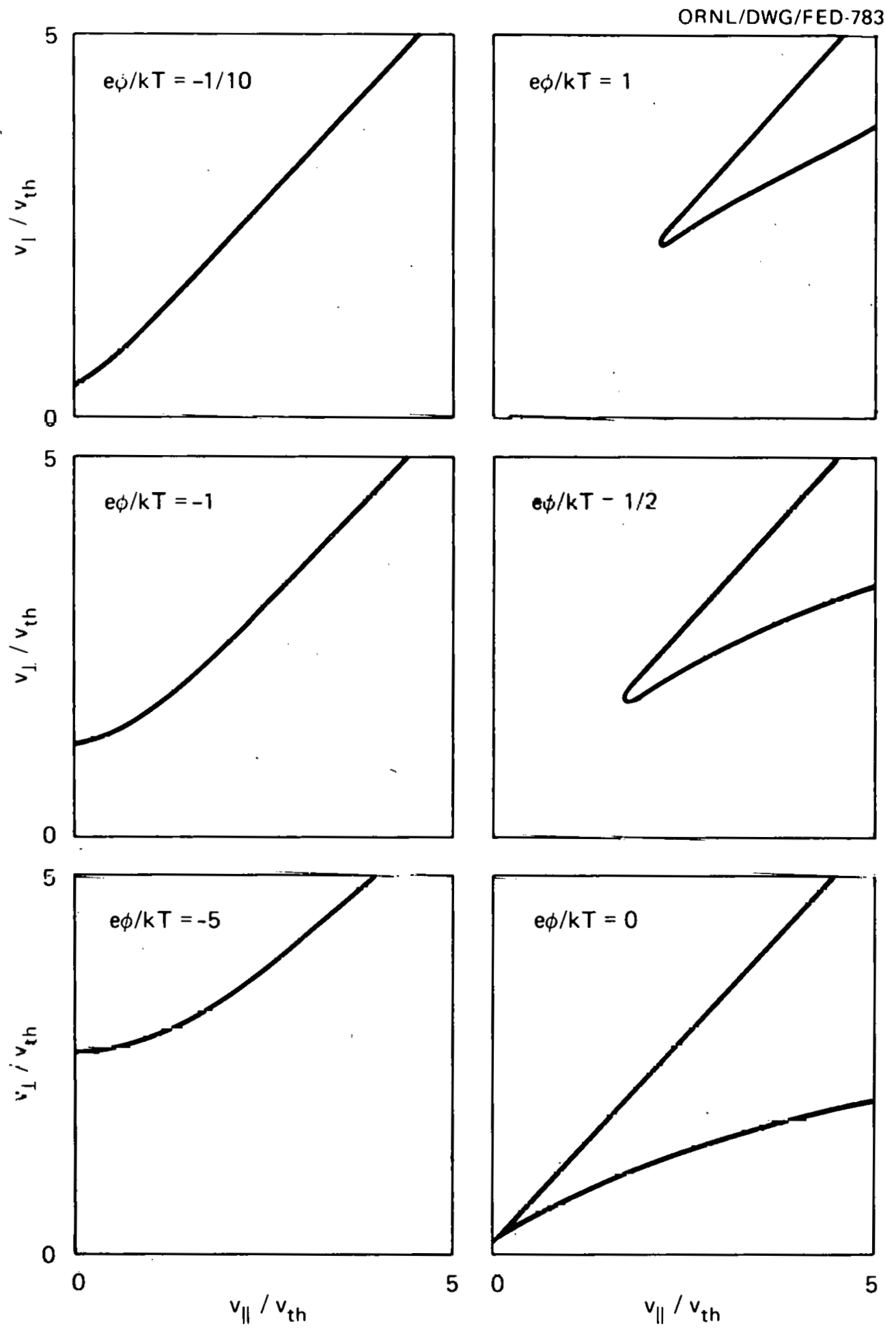

Fig. 3. Contours where the poloidal drift frequency goes to zero vs $v_{\perp} / v_{t h}$ and $v_{\|} / v_{t h}$ as a function of the ambipolar potential. 


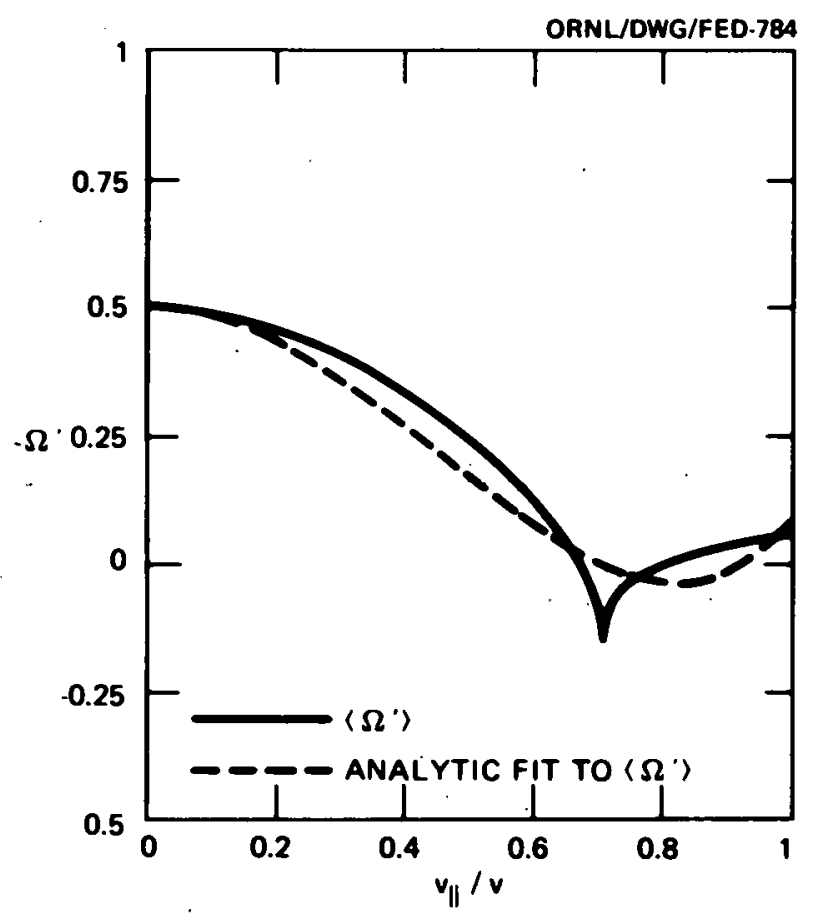

Fig. 4. Dimensionless poloidal drift frequency function $\Omega$ /c vs $\zeta$ (solid line) and approximate fit (dashed line) for EBT-I parameters.

where

$$
\begin{aligned}
R & =\text { major radius, } \\
N & =\text { number of mirror sections, } \\
E_{r} & =\text { ambipolar radial field, } \\
\alpha_{1} & =1 /(1+\alpha), \\
\alpha_{2} & =\alpha /(1+\alpha), \\
\alpha & =\frac{2 \mathrm{eE}}{\mathrm{rkT}}\left(\frac{\mathrm{R}}{\mathrm{N}}\right)^{2}, \\
\Omega_{\mathrm{E} \times \mathrm{B}}^{\prime} & =1, \text { and } . \\
\Omega_{\nabla \mathrm{B}}^{\prime} & \text { is given in Eq. (60). }
\end{aligned}
$$


This choice of normalization is suited to the case of $E_{x}>0$, however, due to the singularity in $\alpha$, and $\alpha_{2}$ at $\alpha=-1$, the above definition is not convenient when $E_{r}<0$. In this case (to be discussed in Sect. V.B) $\Omega_{0}$ is normally taken simply as $N^{2} k T / 2 e B R$.

In the results given in Sect. $V$, we shall assume a parabolic form for the potential $\phi(r)=\phi_{0}\left(1-r^{2} / a^{2}\right)$ so that $\Omega_{0}$ scales as $e \phi_{0} / k T$ instead of $\mathrm{eE}_{\mathrm{r}} \mathrm{a}^{2} / 2 \mathrm{kTr}$. Because the analysis of this paper is local in radius, these two representations of the field are completely interchangeable at any point. The parameterization in terms of $e \phi_{0} / \mathrm{kT}$ is chosen only for convenience.

\section{B. Bounce-Averaged Vertical Drift}

The drift velocity $v_{y}$ caused by the toroldal curvature and associated gradients has been given in Eq. (29). In this form, $v_{y}$ is as yet not bounce averaged since both $v_{\|} / v$ and $\omega_{c}$ are functions of position along a field line. The bounce average of $v_{y}$ is given by the following:

$$
\left\langle\mathrm{v}_{\mathrm{y}}>=\frac{m v^{2}}{2 \mathrm{eR}} \oint \frac{\mathrm{d} \ell}{\mathrm{v}_{\|} \mathrm{B}}\left(1+\frac{\mathrm{v}_{\|}^{2}}{\mathrm{v}^{2}}\right) .\right.
$$

In performing the above average, we will use the magnetic field near the center of the plasma as given in Eq. (48):

$$
B \simeq B_{0}\left[1-\left(\frac{M_{0}-1}{M_{0}+1}\right) \text { cus } k Z\right] \text {. }
$$

For $M_{0}=2$, the bounce average of $v_{y}$ is then:

$$
\begin{array}{r}
<v_{y}>=\frac{2 v_{y m}}{v_{\| m^{\tau}}}\left[\int_{0}^{z_{T}} \frac{d z}{(3-\cos k z) \sqrt{1-k^{2} \sin ^{2} \mathrm{kz} / 2}}\right. \\
\left.\quad+\frac{v_{\| m}^{2}}{v^{2}} \int_{0}^{z_{T} \frac{d z \sqrt{1-k^{2} \sin ^{2} k z / 2}}{3-\cos k z}}\right],
\end{array}
$$


where

$$
\begin{aligned}
v_{y m} & =\frac{m^{2}}{2 e R B_{M}}, \\
B_{M} & =f i e l d \text { at midplane, } \\
z_{T} & =\frac{2}{k} \begin{cases}\sin ^{-1} \kappa^{-1} & \kappa^{2}>1 \\
\pi / 2 & \kappa^{2}<1,\end{cases}
\end{aligned}
$$

and $v_{1 m}, v_{\| m}=v_{1}$ and $v_{\|}$at the midplane, The bounce time $\tau$ has been given in Eq. (57). Substituting this function in Eq. (74) and making a transformation in the integration variable for the $\kappa^{2}>1$ case (to remove singularities), $\left\langle\mathrm{v}_{\mathrm{y}}\right\rangle$ becomes:

$$
\left\langle v_{y}\right\rangle=v_{y m}\left(I_{1}+I_{2}\right)
$$

where

$$
I_{1}= \begin{cases}\frac{1}{k\left(1 / k^{2}\right)} \int_{0}^{\pi / 2} \frac{d x}{\left(1+\kappa^{-2} \sin ^{2} x\right) \sqrt{1-k^{-2} \sin ^{2} x}} & k^{2}>1 \\ \frac{1}{k\left(k^{2}\right)} \int_{0}^{\pi / 2} \frac{d x}{\left(1+\sin ^{2} x\right) \sqrt{1-k^{2} \sin ^{2} x}} & \kappa^{2}<1,\end{cases}
$$

and

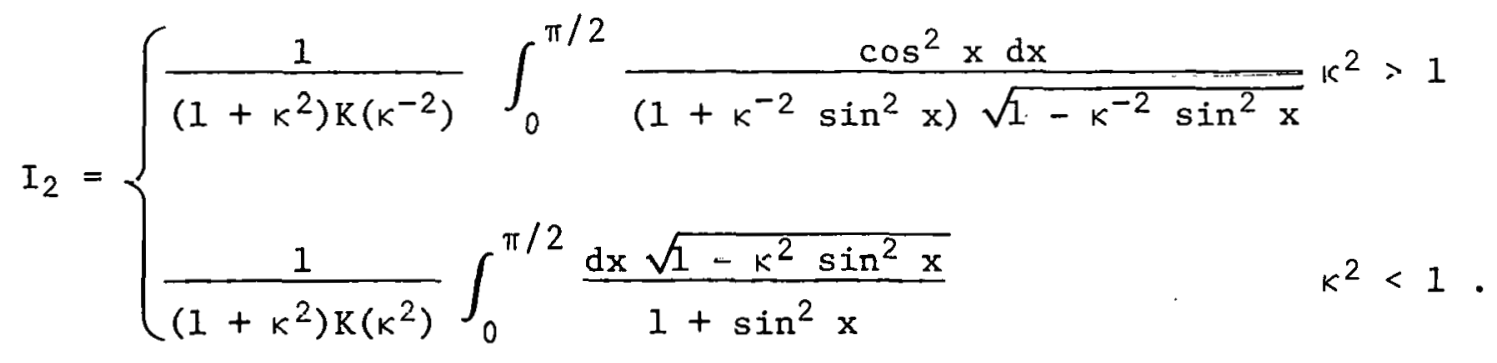


The above integrals have been evaluated numerically over the range $0<v_{\|} / v<1$ and results for the sum $I_{1}+I_{2}$ are presented in Fig. 5a vs $v_{\|} / v$ (solid line). This is compared against the dependence of $v_{y}$ on $v_{\|} / v$ (dashed line $=1+v_{\|}^{2} / v^{2}$ ) before bounce averaging. The dip in the bounce-averaged result at $v_{\|} / v=0.7\left(k^{2}=1\right)$ occurs at the point where orbits are just changing from trapped to passing. Orbits in this particular range of $v_{\|} / v$ spend a significant fraction of their bounce motion in the mirror throats with $v_{\|} \simeq 0$ and thus $v_{y}$ is lowered for such particles. A similar cusped regiun was present in the bounce-averaged rotation frequency $\Omega$ as was mentioned in Sect: IV.A.
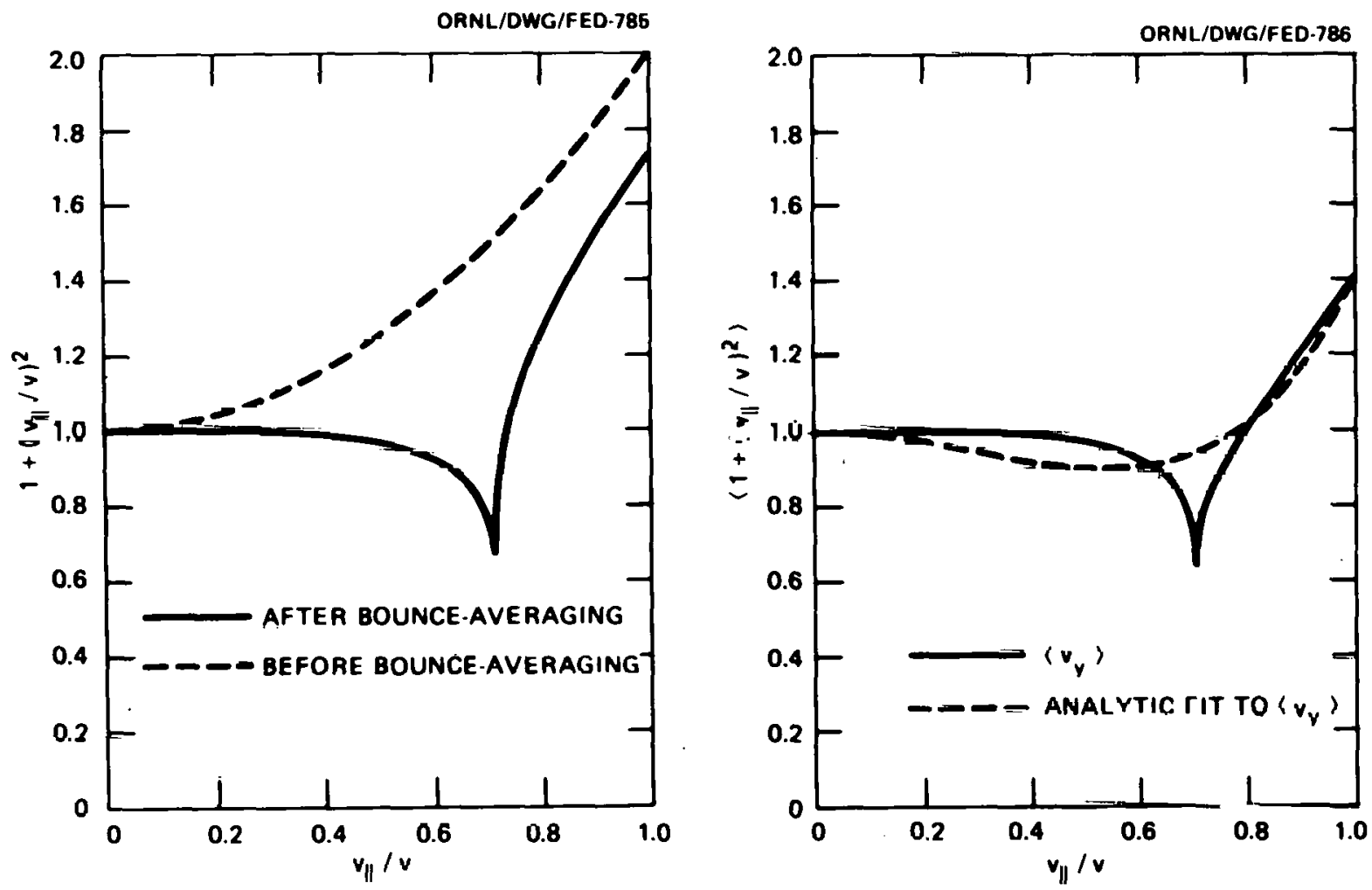

Fig. 5(a). Comparison of bounce-averaged vertical drift $\left\langle v_{y}\right\rangle$ (solid line) with the same drift before bounce averaging (dashed line). (b) Approximate fit (dashed line) to bounce-averaged vertical drift $<v_{y}>$ (solid line). 
An analytic fit to the bounce-averaged drift $\left\langle v_{y}\right\rangle$ has been developed for use in the codes which solve the kinetic equations (discussed in the next section). The fit is given below:

$$
<v_{y}>=v_{y m}\left[1-0.63\left(\frac{v_{\|}}{v}\right)^{2}+1.5\left(\frac{v_{\|}}{v}\right)^{4}\right]
$$

Comparison of this fit against $\left\langle v_{y}\right\rangle$ 1s indicated in Fig. $5 b$. 
THIS PAGE

\section{WAS INTENTIONALLY}

\section{LEFT BLANK}




\section{NUMERICAL SOLUTION PROCEDURES AND RESULTS}

In order to obtain neoclassical transport coefficients, the coupled kinetic Eqs. (34)-(36) of Sect. III must be solved for the distributions $\mathrm{f}_{\mathrm{sn}}, \mathrm{f}_{\mathrm{sT}}$ and appropriate moments taken as indicated by Eqs. (42)-(45). Because the collisinn term considered here is a two-dimensional, integrodifferential operator, and the drift velocities $v_{y}$, and $r \Omega$ are functions having a fair amount of structure with respect to pitch angle and energy, it is difficult to obtain analytic solutions over any realistic range of parameters. As a result, numerical procedures have been utilized and will be discussed in the present section. The transport coefficients given here are specific examples of the methods which have been developed and are not, in their present form, generally applicable over a wide range of experimental devices. Because the particle orbits in a bumpy torus are dependent on particular system parameters such as mirror ratio, distance between mirrors, etc., it has been necessary to use a specific magnetic field model. In this case, the bumpy cylinder model discussed in the previous section has been utilized and system parameters appropriate to the EBT-I device are assumed. We shall not consider modifications of the magnetic field due to finite plasma beta. Also, the results given here have been obtained using a collision operator which is not fully bounce averaged. 'I'his approximation was made in order to facilitate comparison with earlier calculations ${ }^{1,7}$ where such was also the case. In Sect. II, methods and results have been given for bounce averaging the collision Lerm. Also, in the earlier calculations ${ }^{1,7}$ the $v_{y}$ drift was not bounce averaged; results here have a similar approximation. The bounce averaging of $v_{y}$ given in the previous section will be included in future work.

The two solution methods used here are: expansion of the distribution function in a complete set of orthogonal polynomials and finite differcnce iterative soluliuns. The former of these was used first and works adequately for the case where $e \phi_{o} / k T>0$, but fails to converge when $e \phi_{o} / k T<0$. This is related to the structure which can develop in the distribution function when a loss region $(\Omega=0)$ is present at energies near thermal. It might be expected that a functional expansion 
method which is based on a smooth, continuous set of functions will require a very large number of terms in order to represent the steep slopes which can form in a distribution function near such regions. This observation motivated the development of a finite difference code to solve the kinetic equations using the Alternating-Difference-Implicit (ADI) method. The convergence of this method is not as highly dependent on the form of the solution as the functional expansion method is.

\section{A. Functional Expansion Method}

Functional representations have been used extenslvely in plasma kinetic theory. 25-27 More recently, such methods have also been employed in solving the Boltzmann equation in collisfonal plasma model studies of tokamak neoclassical transport and drift wave instabilities. 28-31 Our approach uses a similar representation in velocity space as in Ref. 30 .

This solution method consists of expanding the first order distribution function in terms of the generalized Laguerre polynomials in $v$ and Legendre polynomials in pitch angle, times the Maxwellian function.

$$
\pm_{1}=\sum_{n, l}\left|f_{1}\right\rangle_{n \ell} \psi_{n \ell} .
$$

where

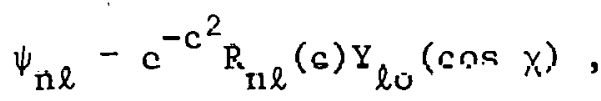

$$
\begin{aligned}
& c^{2}=m v^{2} / 2 k T, \\
& \mathrm{~K}_{\mathrm{n} \ell}=\mathrm{N}_{\mathrm{n} \ell} \mathrm{c}^{\ell} \mathrm{L}_{(\mathrm{n}-\ell) / 2}^{\ell+1 / 2}\left(\mathrm{c}^{2}\right),
\end{aligned}
$$

and

$$
\begin{aligned}
N_{n \ell} & =\text { normalization factor } \\
& =\left[\frac{2}{\pi^{1 / 2}} \int_{0}^{\infty} c^{2} d c \int_{-1}^{1} \mathrm{~d}(\cos x) \mathrm{e}^{-c^{2}} \mathrm{R}_{\mathrm{n} \ell}^{2}(\mathrm{c}) \mathrm{Y}_{\ell 0}^{2}(\cos x)\right]^{-1 / 2} .
\end{aligned}
$$


Solution of the kinetic Eqs. (34)-(36) then involves substitution of the above expansion for $f_{s n}, f_{c n}, . ., f_{s e}, f_{c e}$ and inversion of the resulting matrix equations for the coefficient vectors $\left|f_{s n}\right\rangle$, $\left|\mathrm{f}_{\mathrm{cn}}{ }, . . .,\right| \mathrm{f}_{\mathrm{se}}{ }, \mid \mathrm{f}_{\mathrm{ce}}{ }^{\rangle}$. The particular functions given in Eq. (79) have been chosen since they are exact eigenfunctions of a simplified model collision operator which has been given in Ref. 32. These functions also have the convenient property that the lowest order term is the Maxwellian.

As an example of the functional expansion method, the procedure for solving the $\mathrm{f}_{\mathrm{sn}}, \mathrm{f}_{\mathrm{cn}} \mathrm{Eq} .(34)$ is outlined below. We shall assume steady state and abbreviate the collision terms as $\mathrm{Cf}_{\mathrm{sn}}$ and $\mathrm{Cf} \mathrm{cn}$. Also, brackets indicating bounce averages are dropped for simplicity.

$$
\left\{\begin{array}{l}
\mathrm{Cf}_{\mathrm{sn}}+\Omega \mathrm{f}_{\mathrm{cn}}=\mathrm{v}_{\mathrm{y}} \mathrm{f}_{\mathrm{o}} \\
\mathrm{Cf_{ \textrm {cn } }}-\Omega \mathrm{f}_{\mathrm{sn}}=0 .
\end{array}\right.
$$

In order to cast these equations into a convenient form for numerical solution, the following dimensional factors are defined.

$$
\begin{aligned}
& \Omega=\Omega_{0} \Omega^{\prime} \quad\left[\Omega_{0}\right. \text { was given in Eq. (71)] . } \\
& v_{y}=v_{0} v_{y}^{\prime} ; \quad v_{0}=2 k T / e B_{M} R \\
& C=\nu_{0} C^{\prime} \\
& \nu_{0}=\left(\mathrm{nl} / \pi^{3 / 2} \alpha^{3}\right) ; \quad \Gamma=4 \pi^{2} \mathrm{e}^{4} \ln \Lambda / \mathrm{m}^{2} . \\
& \mathrm{f}_{\mathrm{o}}=\left(\mathrm{n} / \pi^{3 / 2} \alpha^{3}\right) \mathrm{f}_{\mathrm{o}}^{\prime} ; \quad \alpha=\sqrt{2 \mathrm{kT} / \mathrm{m}} . \\
& \zeta=\cos x=v_{\|} / v
\end{aligned}
$$




$$
\begin{aligned}
& \left.f_{s n}=\left(\frac{n v_{o}}{\pi^{3 / 2} \alpha^{3} v_{0}}\right) f_{s n_{0}^{\prime}}^{\prime} \quad \text { (the same factor applies to } f_{s T} \text { and } f_{s e}\right) . \\
& \mathrm{f}_{\mathrm{cn}}=\left(\frac{\mathrm{n} \Omega_{\mathrm{o}} \mathrm{v}_{\mathrm{o}}}{\pi^{3 / 2} \alpha^{3} v_{\mathrm{o}}^{2}}\right) \mathrm{f}_{\mathrm{cn} \mathrm{n}_{\mathrm{o}}}^{\prime} \text { (the same factor applies to } \mathrm{f}_{\mathrm{cT}} \text { and } \mathrm{f}_{\mathrm{ce}} \text { ). }
\end{aligned}
$$

Note from the above definitions that:

$$
\begin{aligned}
& f_{0}^{\prime}=e^{-c^{2}}, \\
& v_{y}^{\prime}=c^{2}\left(1+10^{2}\right) / 2,
\end{aligned}
$$

and

$$
E^{\prime}=E / k T=c^{2}
$$

Also, the coupled kinetic Eq. (80) becomes:

$$
\begin{aligned}
& C^{\prime} f_{\text {sn }}^{\prime}+\lambda \Omega^{\prime} f_{c n}^{\prime}=v_{y}^{\prime}, \\
& C^{\prime} f_{c n}^{\prime}-\Omega^{\prime} f_{\text {sn }}^{\prime}=0,
\end{aligned}
$$

where $\lambda=\left(\Omega_{0} / v_{0}\right)^{2}$. $f_{s n}^{\prime}, f_{c n}^{\prime}$, and $v_{y}^{\prime}$ are then expanded using the functions given in Eq. (79):

$$
\begin{aligned}
f_{\theta, c n}^{\prime} & =\sum_{n=0}^{N} \sum_{\ell=0}^{n} R_{L i \ell}(c) Y_{\ell, 0}(\zeta)\left|f_{s, c n}^{\prime}\right\rangle_{n \ell} ; \\
v_{y}^{\prime} & =\sum_{n=0}^{N} \sum_{\ell=0}^{n} R_{n \ell}(c) Y_{\ell v}\left(\zeta_{2}\right)\left|v_{y}^{\prime}\right\rangle_{n \ell} .
\end{aligned}
$$


Substituting expansions given in Eq. (82) into Eq. (81), and taking moments with

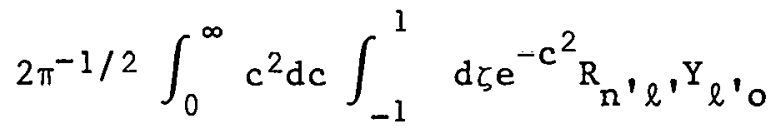

results in the following matrix equations.

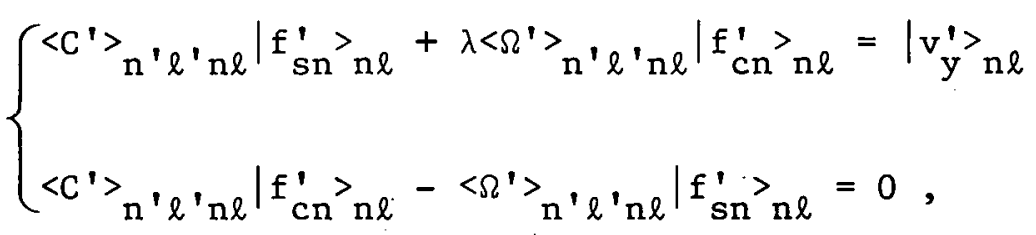

where $\left\langle\Omega^{\prime}\right\rangle_{n^{\prime}} \ell^{\prime} n \ell$ and $\left\langle C^{\prime}\right\rangle_{n^{\prime}} \ell^{\prime} n \ell$ are the matrix elements of the poloidal rotation frequency and collision operator. These are calculated in Appendixes $A$ and $B$, respectively, using the fit to $\Omega^{\prime}$ given in Eq. (70) and the Landau form of the collision operator.

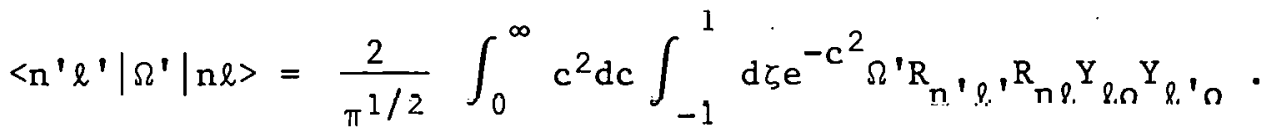

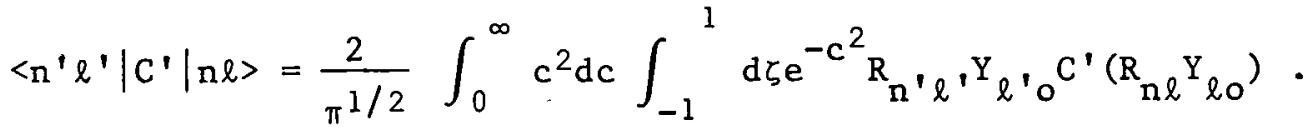

The solution of Eq. (83) may be written as follows.

$$
\begin{aligned}
& \left|\mathrm{f}_{\mathrm{cn}}^{\prime}\right\rangle=\left(\lambda\left\langle\Omega^{\prime}\right\rangle+\left\langle\mathrm{C}^{\prime}\right\rangle\left\langle\Omega^{\prime}\right\rangle^{-1}\left\langle\mathrm{C}^{\prime}\right\rangle\right)^{-1}\left|\mathrm{v}_{\mathrm{y}}^{\prime}\right\rangle . \\
& \left|\mathrm{f}_{\mathrm{sn}}^{\prime}\right\rangle=\left\langle\Omega^{\prime}\right\rangle^{-1}\left\langle\mathrm{C}^{\prime}\right\rangle\left(\lambda\left\langle\Omega^{\prime}\right\rangle+\left\langle\mathrm{C}^{\prime}\right\rangle\left\langle\Omega^{\prime}\right\rangle^{-1}\left\langle\mathrm{C}^{\prime}\right\rangle\right)^{-1}\left|v_{\mathrm{y}}^{\prime}\right\rangle .
\end{aligned}
$$


Solutions for the distributions $\mid f_{\mathrm{CT}^{\prime}}^{\prime}{ }^{\prime}$ and $\mid \mathrm{f}_{\mathrm{ST}^{\prime}}^{\prime}{ }^{\prime}$ of $\mathrm{Eq}$. (35) are of exactly the same form as Eqs. (86) and (87) except that $\left|v_{y}^{\prime}\right\rangle$ is replaced by $\left|v_{y}^{\prime}(E / k T-3 / 2)\right\rangle$. The six transport coefficients are then obtained by taking the following products of the vectors, $\left|f_{s n}^{\prime}\right\rangle,\left|f_{s T}^{\prime}\right\rangle,\left|v_{y}^{\prime}\right\rangle$, and $\left|E^{\prime} v_{y}^{\prime}\right\rangle$ :

$$
\begin{aligned}
D_{n} & =-\frac{v_{0}^{2}}{2 v_{0}}\left\langle v_{y}^{\prime} \mid f_{s n}^{\prime}\right\rangle, \\
D_{T} & =-\frac{v_{v}^{2}}{2 v_{0}^{\prime k T}}\left\langle v_{y}^{\prime}\right| f_{s T^{\prime}}^{\prime}, \\
K_{n} & =-\frac{v_{0}^{2} k T}{2 v_{0}}\left\langle E^{\prime} v_{y}^{\prime} \mid f_{s n}^{\prime}\right\rangle, \\
& \ddots \\
K_{T} & =-\frac{v_{0}^{2}}{2 v_{0}}\left\langle v_{y}^{\prime} \mid f_{s T^{\prime}}^{\prime}\right\rangle, \\
\mu_{n} & =\frac{e}{k T} D_{n},
\end{aligned}
$$

and

$$
\mu_{E}-\frac{\mathrm{e}}{\mathrm{kT}} \mathrm{k}_{\mathrm{n}}
$$

To summarize, the basic steps involved in the functional expansion method are: (1) calculate the matrix elements for the collision operator and the pololdal rotation frcquency $s^{\prime}$, (2) perfuim the utd trix Inversions and multiplications of $\mathrm{Eq}$. (87) to obtain $\left|\mathrm{f}_{\mathrm{sn}}^{\prime}\right\rangle$ (and an analogous equation for $\left.\left|f_{s T}^{\prime}\right\rangle\right)$, and (3) take the vector products of Eq. (88) to obtain the transport coefficients.

This procedure has been automated into a computer code which is highly flexible with respect to the number of trial functions used in 
the representation. Up to 78 terms in the expansions of Eq. (82) may be retained (corresponding to $n=22,1=22$ ). By varying the number of terms in the expansion, the convergence properties may be checked. It has been found that relatively few terms are required when the ambipolar field is large (e $\phi_{0} / \mathrm{kT}>1$ ), but significantly more are necessary when $0<\mathrm{e} \phi_{\mathrm{o}} / \mathrm{kT}<1$. This feature is illustrated in Figs. 6 and 7 where the density diffusion coefficients are plotted against $\nu_{0} / \Omega_{0}$ and as a function of the number of terms used in the expansions. Results are given for $e \phi_{o} / k T=0$ (Fig. 6) and $e_{o} / k T=1$ (Fig. 7); the lines plotted there correspond to using $6,10,15, . . ., 78$ functions in the representation (i.e., $\mathfrak{n}=4,6,8, \ldots . ., 22$ ). The other transport coefficients exhibit a similar behavior with respect to varying the number of functions. As may be seen, $D_{n}$ is monotone increasing as the number of basis functions is augmented. This feature is a result of the fact that $D_{n}$ may be cast into the form of a variational principle which is extremized as the representation becomes more exact (derived in Appendix $C$ ). The slower convergence behavior obtained for $e_{0} / k T=0$ is related to the presence of regions with $\Omega=0$ at velocities in the thermal range, as discussed in sect. IV. As e $\phi_{0} / k T$ is increased $\left(e \phi_{0} / k T>>1\right)$, such regions are moved out to high velocities (see Fig. 3) and affect only a very insignificant fraction of the distribution. As was mentioned earlier, convergence was not obtained when $e_{o} / \mathrm{kT}<0$ (except for large negative values, e $\phi_{0} / \mathrm{kT}<-10$ ) even with up to 78 functions retained in the expansion.

In Fig. 8(a-d), the distribution function, $f_{s n}$ (used in calculating $\mathrm{D}_{\mathrm{n}}$ and $\left.\mathrm{K}_{\mathrm{n}}\right)$, is plotted vs the velocity space variables $\zeta(=\cos x)$ and $|\overrightarrow{\mathrm{v}}| / v_{\text {th }}$ for $\nu_{0} / \Omega_{0}=0.1$ (using 78 functions). As may be seen, there is a peak near $\mathrm{v} / \mathrm{v}_{\mathrm{th}} \simeq 1$ and at $\mathrm{v}_{\|} / \mathrm{v} \simeq 1$, the height of which is strongly dependent on $e \phi_{o} / k T$. This feature is related to the fact that parciclca scattering into the region of $\mathrm{v}_{\|} / \mathrm{v} \simeq 1$ have the largest shifts away from the center of the torus and thus the largest neoclassical step sizes. Also, the $\Omega=0$ region is located near $v_{\|} / v=1$ for $0<e \phi_{0} / k T<1$. 


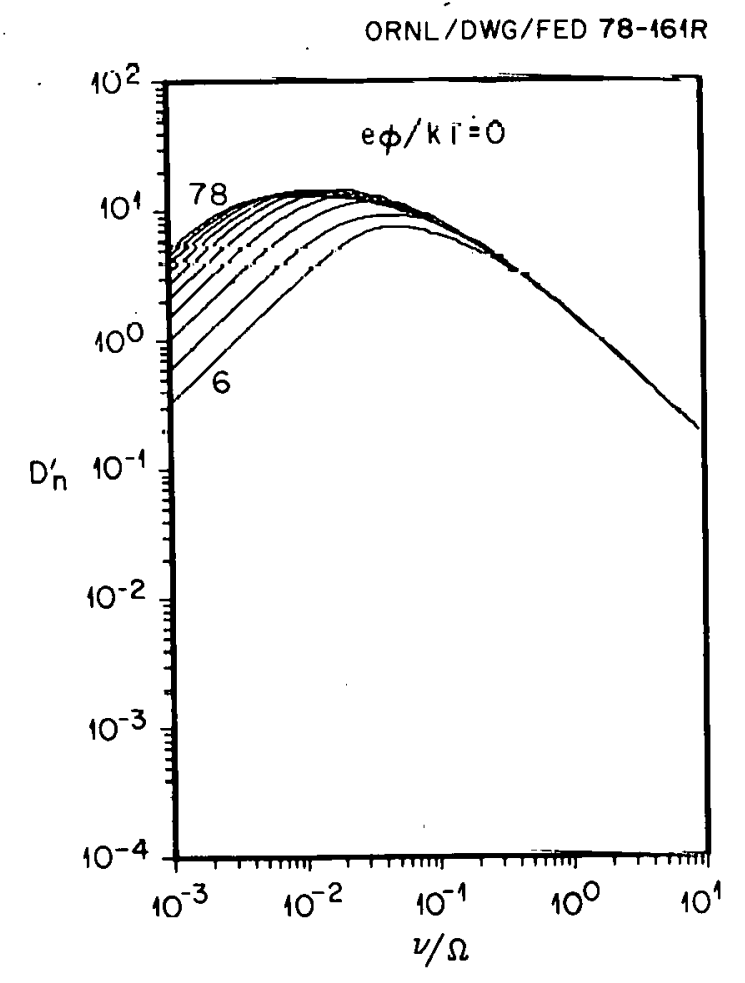

Fig. 6. Convergence properties of functiona1. representation method for $D_{n}$ transport coefficient vs $\nu_{0} / \Omega_{0}$ for $e \phi_{0} / k^{\prime} l=0$. Resulcs alt plotted using $6,10,15, . . ., 78$ functione.

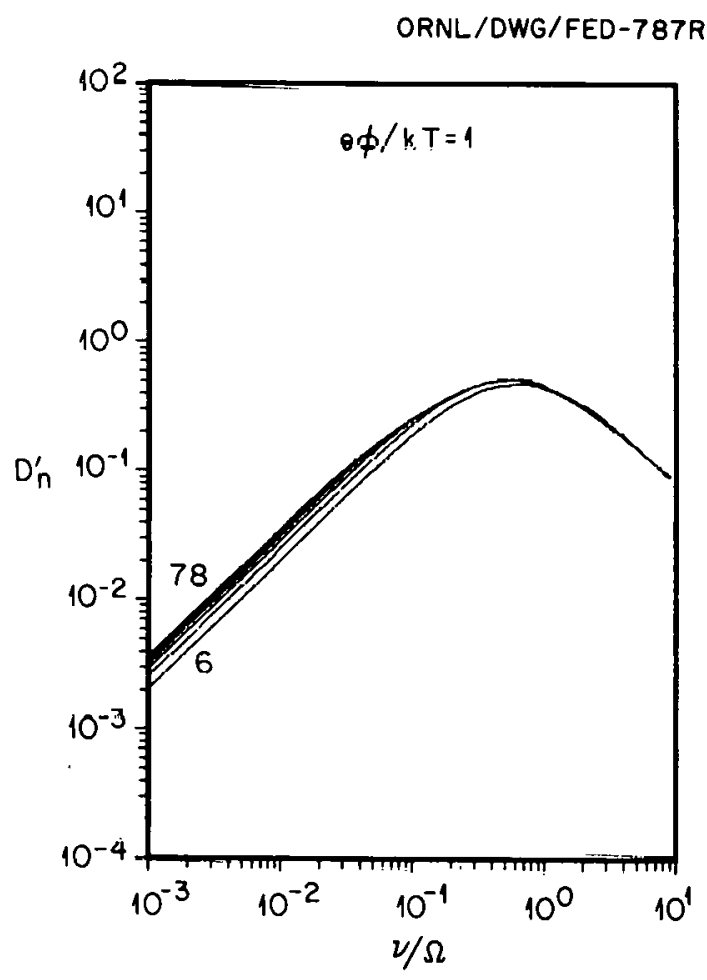

Fig. 7. Convergence properties of functional representation method For $D_{n}$ trancport coefficient vc $\nu_{0} / \Omega_{0}$ for o $\phi_{0} / k \cdot T=1$. Results are plotted using $6,10,15, . . ., 78$ functions. 


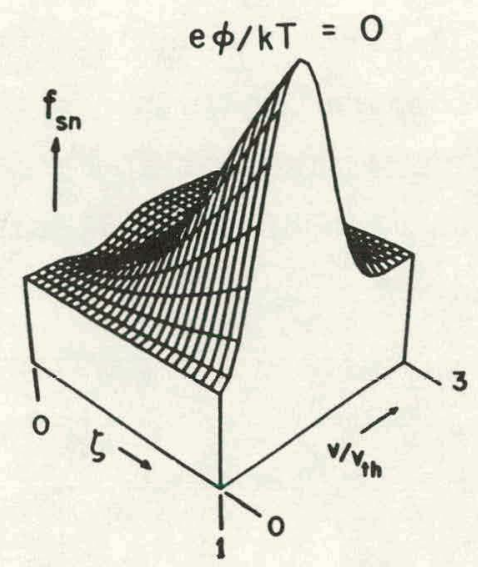

ORNL/DWG/FED-788
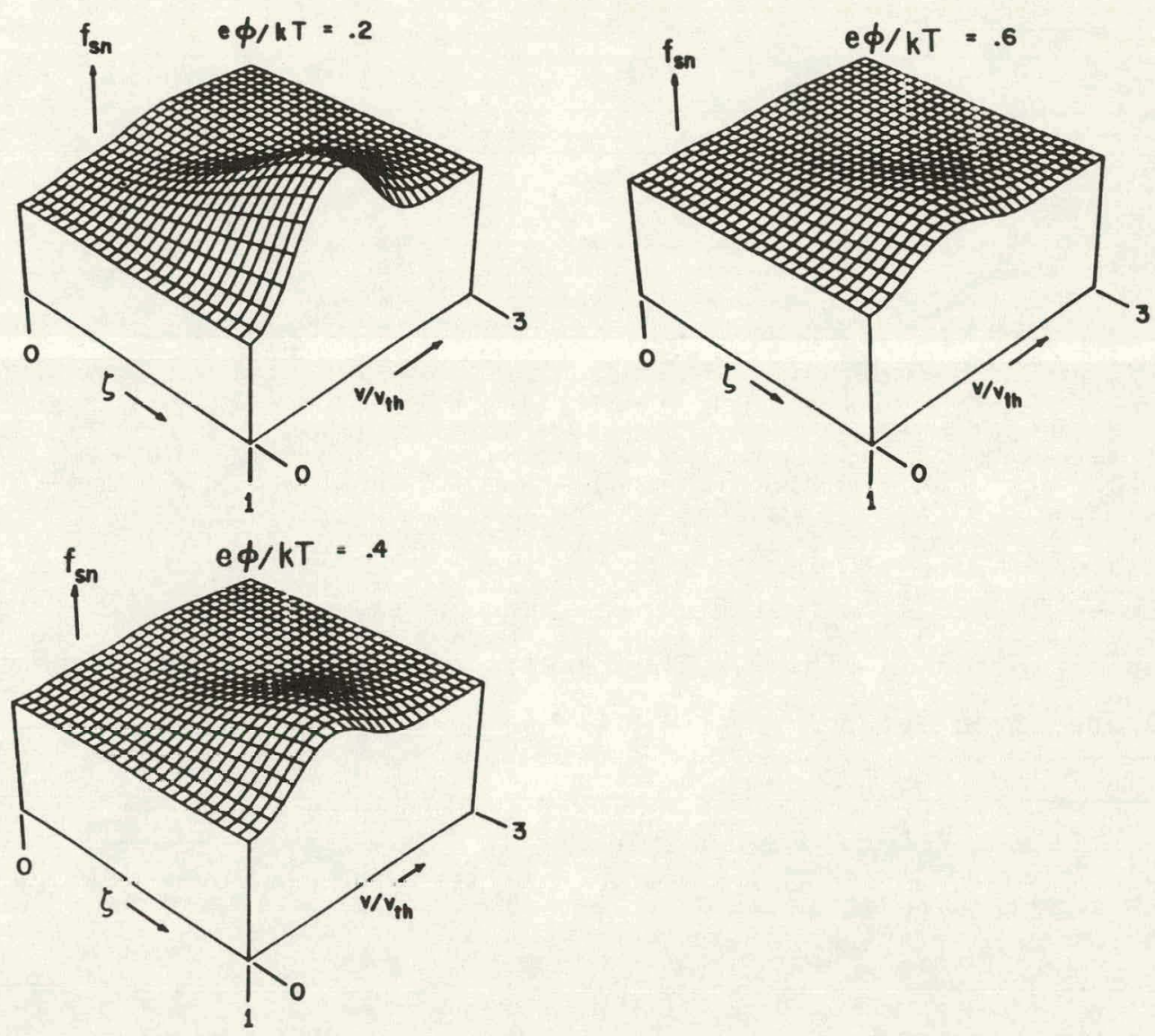

Fig. 8. $\mathrm{f}_{\mathrm{sn}}^{\prime}$ distribution function $v s \zeta$ and $v / v_{\text {th }}$ for $\mathrm{e} \phi_{\mathrm{o}} / \mathrm{kT}=0,0.2,0.4,0.6$, and $\nu_{\mathrm{o}} / \Omega_{\mathrm{o}}=0.1$. 
The full set of neoclassical transport coefficients, $D_{n}, D_{T}, K_{n}, K_{T}$, are plotted in Fig. 9 vs the parameters $\nu / \Omega_{0}$ and $e \phi_{0} / k T . \mu_{n}$ and $\mu_{E}$ have been related to $\mathrm{D}_{\mathrm{n}}$ and $\mathrm{K}_{\mathrm{n}}$ by Eq. (46). Results are presented for $\mathrm{e} \phi_{\mathrm{o}} / \mathrm{kT}=$ $0,1 / 2,1,2,4$, and $>>1$. The latter is compared against earlier work by Kovrizhnykh ${ }^{1,7}$ (indicated by triangles) and also for the e $\phi_{0} / \mathrm{kT}>1$ case. The functions plotted here have been made dimensionless and are related to the actual transport coefficients by the following factors.

$$
\begin{aligned}
& \mathrm{D}_{\mathrm{n}}=\frac{\mathrm{v}_{\mathrm{o}}^{2}}{2 \Omega_{\mathrm{o}}} \mathrm{D}_{\mathrm{n}}^{\prime}, \\
& \mathrm{D}_{\mathrm{T}}-\frac{\mathrm{nv_{ \textrm {o } } ^ { 2 }}}{2 \Omega_{\mathrm{o}} \mathrm{kT}} \mathrm{D}_{\mathrm{T}}^{\prime}, \\
& \mathrm{K}_{\mathrm{n}}=\frac{\mathrm{kTv_{ \textrm {o } } ^ { 2 }}}{2 \Omega_{\mathrm{o}}} \mathrm{K}_{\mathrm{n}}^{\prime},
\end{aligned}
$$

and

$$
\mathrm{K}_{\mathrm{T}}=\frac{\mathrm{nv}_{\mathrm{o}}^{?}}{2 \Omega_{\mathrm{o}}} \mathrm{K}_{\mathrm{T}}^{\prime} \text {, }
$$

where $v_{0}=2 k T / e M_{M} R, \Omega_{0}$ was defined in Eq. (71), and $D_{n}^{\prime}, D_{T}^{\prime}, K_{n}^{\prime}, K_{T}^{\prime}=$ functions plotted in Fig. 9. The results of Kovrizhnykh ${ }^{1,7}$ presented in Fig. 9 are given by:

$$
\begin{aligned}
& D_{\mathrm{n}}^{\prime}=\frac{1}{12} \frac{\nu_{\mathrm{o}} / \Omega_{0}}{1+\left(\nu_{\mathrm{o}} / \Omega_{0}\right)^{2}}, \\
& \mathrm{D}_{\mathrm{T}}^{\prime}=\frac{1}{6} \frac{\nu_{\mathrm{o}} / \Omega_{0}}{1+\left(\nu_{\mathrm{o}} / \Omega_{0}\right)^{2}},
\end{aligned}
$$


ORNL/DWG/FED-789R

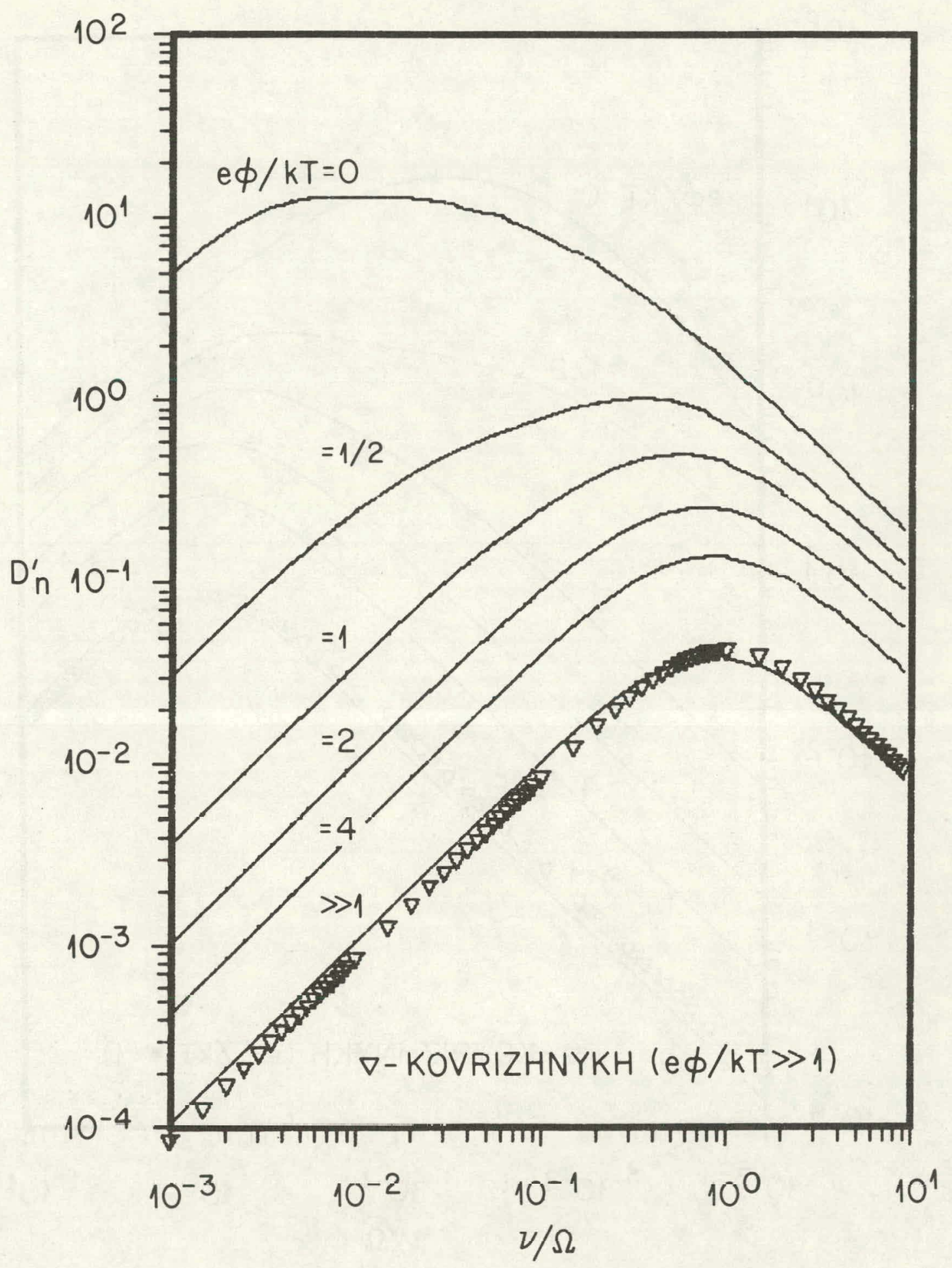

Fig. 9(a). $D_{n}^{\prime}$ bumpy torus neoclassical transport coefficients vs $\nu_{0} / \Omega_{0}$ and as a function of the ambipolar potential. 
ORNL / DWG/FED-7810R

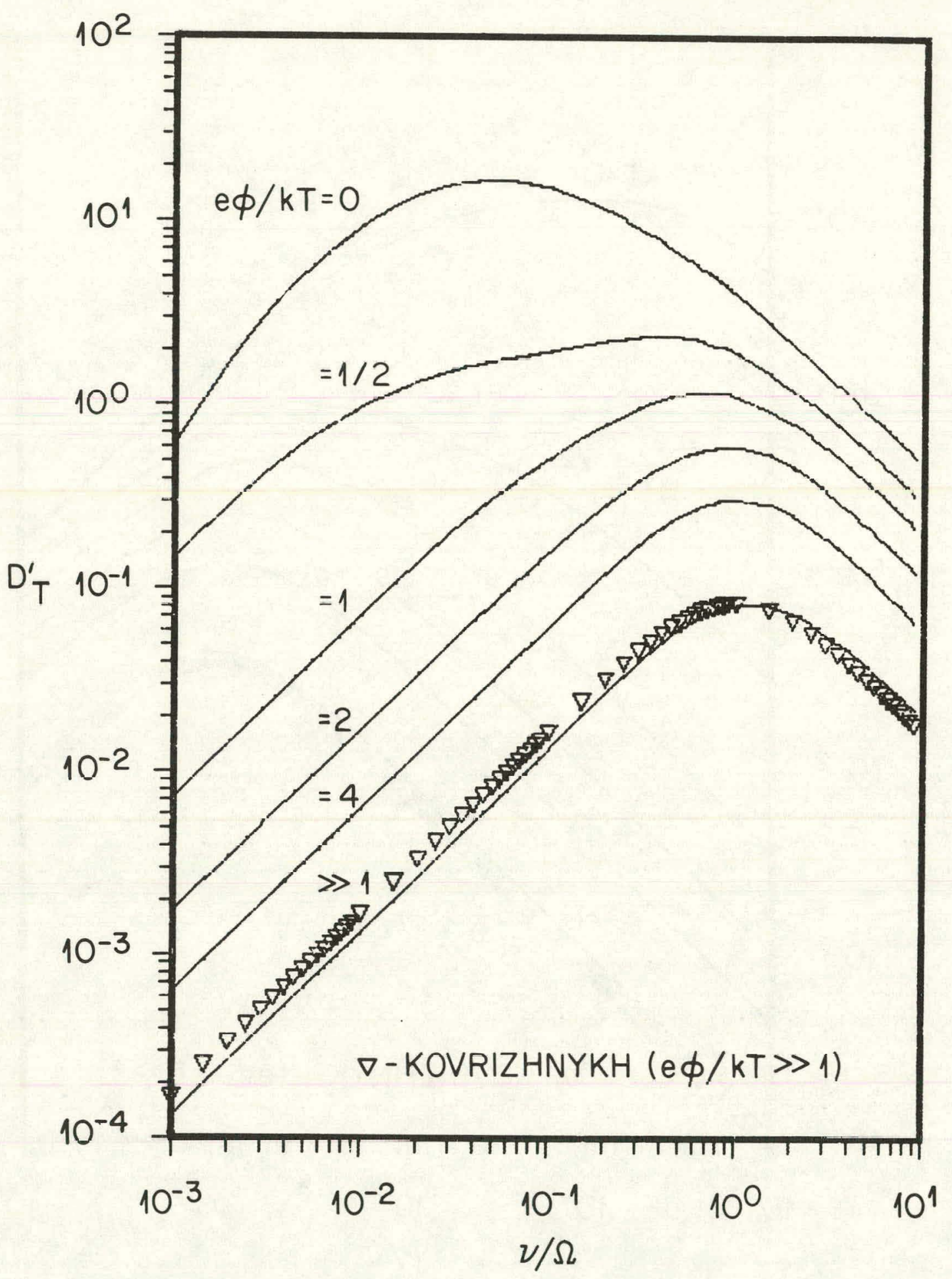

Fig. 9(b). $D_{T}^{\prime}$ bumpy torus neoclassical transport coefficients vs $\nu_{0} / \Omega_{0}$ and as a function of the ambipolar potential. 
ORNL / DWG/FED-7811R

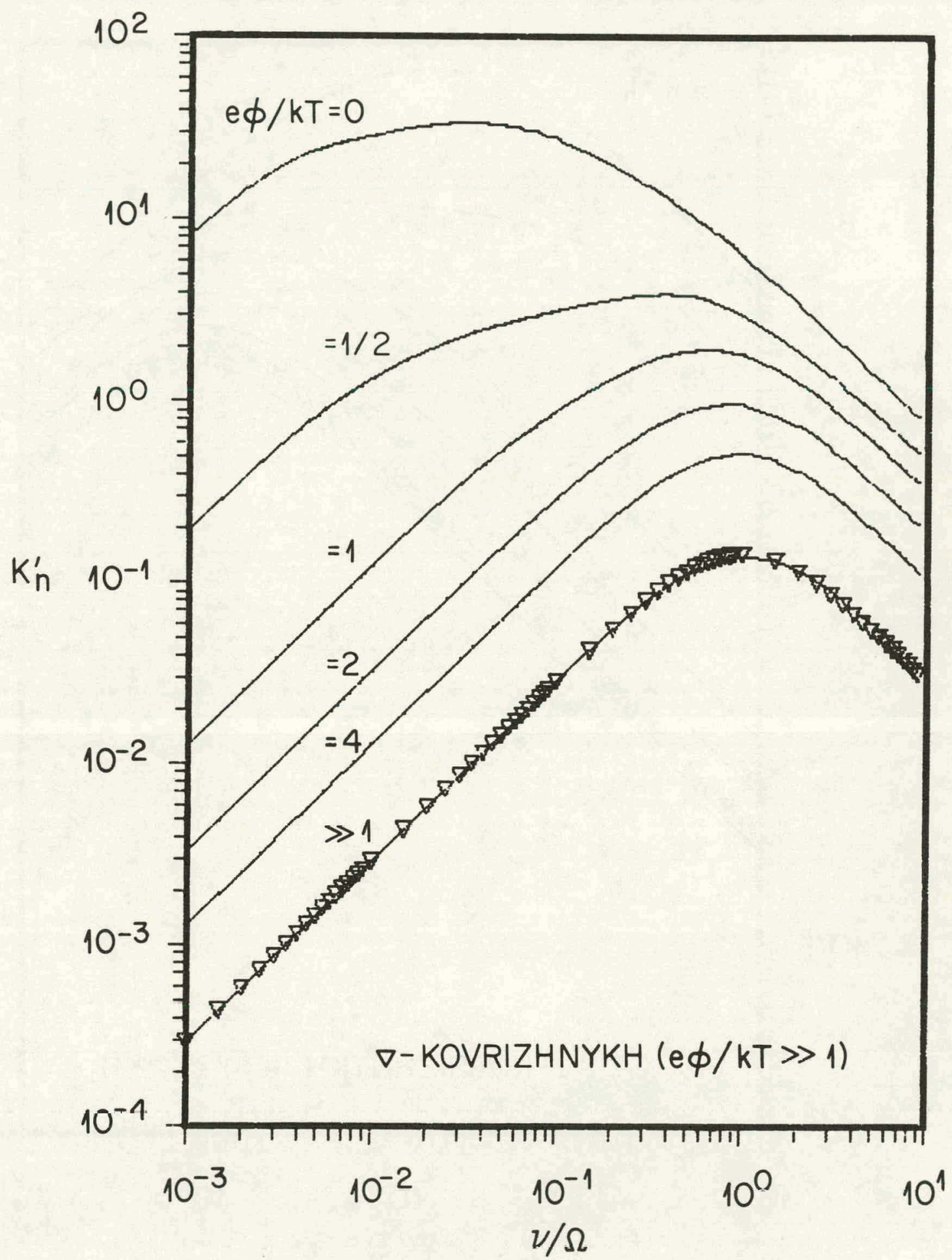

Fig. 9(c). $K_{n}^{\prime}$ bumpy torus neoclassical transport coefficients vs $\nu_{0} / \Omega_{0}$ and as a function of the ambipolar potential. 


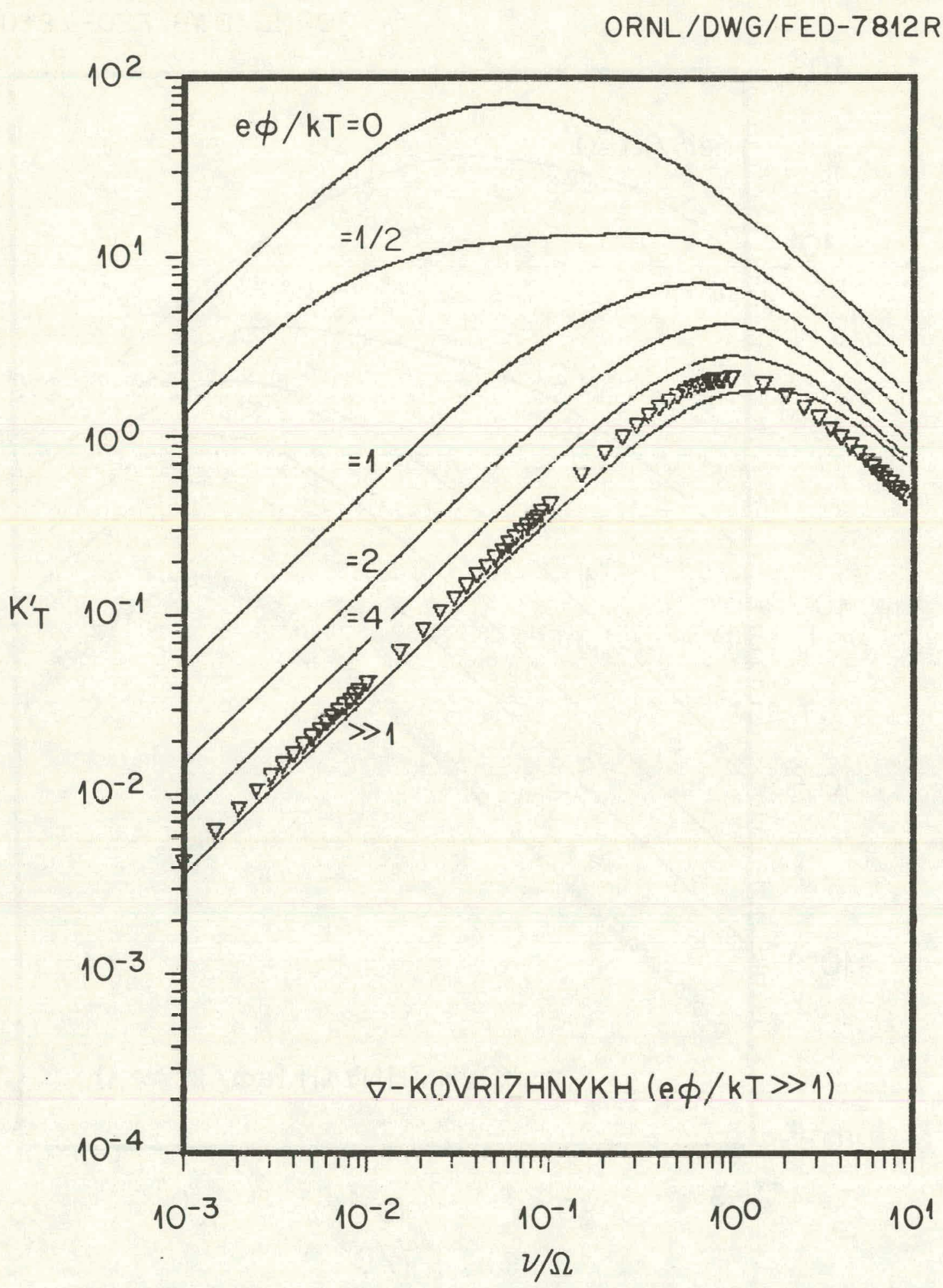

Fig. $9(\mathrm{~d}) \cdot \mathrm{K}_{\mathrm{T}}^{\prime}$ bumpy torus neoclassical transport coefficients vs $\nu_{0} / \Omega_{0}$ and as a function of the ambipolar potential. 


$$
\mathrm{K}_{\mathrm{n}}^{\prime}=\frac{7}{24} \frac{\nu_{0} / \Omega_{0}}{1+\left(\nu_{0} / \Omega_{0}\right)^{2}},
$$

and

$$
\mathrm{K}_{\mathrm{T}}^{\prime}=\frac{101}{24} \frac{\nu_{\mathrm{o}} / \Omega_{\mathrm{o}}}{1+\left(\nu_{\mathrm{o}} / \Omega_{\mathrm{o}}\right)^{2}}
$$

Figure 9 shows that the transport coefficients have a strong dependence on the size of the ambipolar field. This is related to the way in which it controls the location of the $\Omega=0$ loss regions in velocity space (discussed in Sect. IV). In the large field limit [Eq. (90)] where such regions are not present, the transport coefficients scale as $1 / \mathrm{E}_{\mathrm{r}}^{2}$ because this is the dependence of the step size squared $\left[\left(v_{y} / \Omega\right)^{2}\right]$ on $E_{r}$. For finite fields, an even stronger dependence is present due to the influence of the $\Omega=0$ zones on transport.

\section{B. Finite Difference ( $A D I)$ Method}

The ADT method has heen used extensively 19,20 in solving FokkerPlanck kinetic equations for mirror confined plasmas. As was mentioned earlier, this technique has been utilized in EBT transport to obtain results for inwardly pointing ambipolar fields (i.e., e $\phi_{0} / \mathrm{kT}<0$ ); it has also been useful in checking results obtained using the functional expansion method for $\mathrm{e} \phi_{\mathrm{o}} / \mathrm{kT}>0$.

Since the ADI method is designed to solve time-dependent problems, the time derivatives in Eqs. (34)-(36) are retained and the solution is followed until steady-state conditions are achieved. Due to the fact that each of the coupled Eqs. (34)-(36) behave like slowly damped harmonic oscillators, it has been necessary to add artificial damping terms to these equations which go to zero in the steady state. For example, the coupled time-dependent equations for $\mathrm{f}_{\mathrm{sn}}^{\prime}$ and $\mathrm{f}_{\mathrm{cn}}^{\prime}$ are: 


$$
\begin{aligned}
& \frac{\partial f_{s n}^{\prime}}{\partial t^{\prime}}-C^{\prime} f_{s n}^{\prime}-\lambda \Omega^{\prime} f_{c n}^{\prime}=-v_{y}^{\prime} ; \\
& \frac{\partial f_{c n}^{\prime}}{\partial t^{\prime}}-C^{\prime} f_{c n}^{\prime}+\Omega^{\prime} f_{s n}^{\prime}=0,
\end{aligned}
$$

where $t^{\prime}=t \nu_{0}$. In order to obtain more rapidly damped solutions, the following terms have been added.

$$
\left\{\begin{array}{l}
\frac{\partial f_{s n}^{\prime}}{\partial t^{\prime}}+\alpha_{c} \frac{\partial f_{c n}^{\prime}}{\partial t^{\prime}}-C^{\prime} f_{s n}^{\prime}-\lambda \Omega^{\prime} f_{c n}^{\prime}=-v_{y}^{\prime} \\
\frac{\partial f_{c n}^{\prime}}{\partial t^{\prime}}+\alpha_{s} \frac{\partial f_{s n}^{\prime}}{\partial t^{\prime}}-C^{\prime} f_{c n}^{\prime}+\Omega^{\prime} f_{s n}^{\prime}=0 .
\end{array}\right.
$$

Here $\alpha_{s}$ and $\alpha_{c}$ are adjustable parameters which may be used to control the frequency and damping rate of the oscillations in the solution. An example of the influence of these parameters is indicated in Fig. 10 where $D_{n}^{\prime}$ is plotted vs time for various values of $\alpha_{s}$ with $\alpha_{c}=0$. For simplicity, the collision operator used here is the constant coefficient Fokker-Planck operator as given in Ref. 32 and will be indicated below. The oscillations are centered about the same equilibrium value in all cases, but converge only in the case where $\alpha_{s}>0$ and diverge where $\alpha_{s}<0$.

The coupled Eq. (93) has been solved on a two-dimensional grid in velocity space with equidistant spacing in $X$, the pltch angle, and a stretched coordinate spacing in $v$, velocity. This stretching is normally arranged so that more grid points are concentrated at the lower velocities. The finite difference equations and boundary conditions are similar to those given in Ref. 19 except that a simplified collision operator has been used for cases presented in this paper. In solving the finite difference equations, a banded matrix solver has been employed. 01d and new solutions are averaged every two half-time steps with a weighting chosen to provide underrelaxation. 

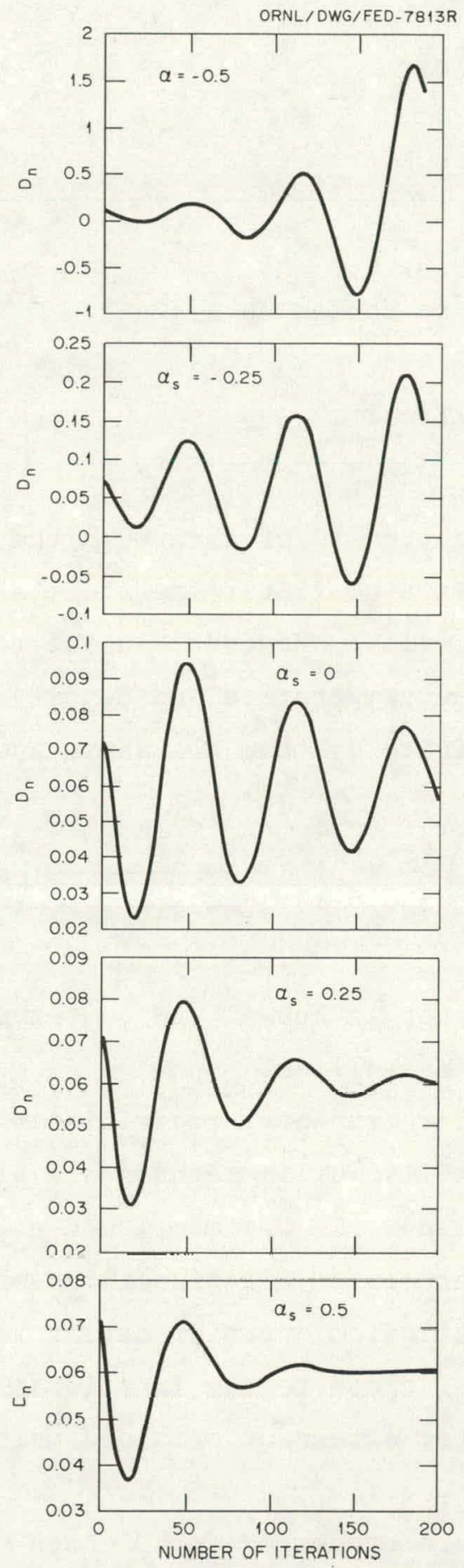

Fig. 10. Convergence properties of $\mathrm{ADI}$ finite difference solution of Eq. (93) using the constant coefficient model collision operator ${ }^{32}$ vs the damping factor $\alpha_{\mathrm{S}}$. These results are for $\nu_{0} / \Omega_{0}=1$ and $\mathrm{e} \phi_{0} / \mathrm{kT}=3$. 
The collision operator used in these calculations is that given by Dougherty: 32

$$
C f=\frac{\partial}{\partial \vec{v}} \cdot(\vec{A} f)+\frac{\partial^{2}}{\partial \vec{v}^{2}}:(\stackrel{\leftrightarrow}{D} f)
$$

The coefficients $\vec{A}$ and $\stackrel{\leftrightarrow}{D}$ are chosen as:

$$
\vec{A}=\nu_{u} \vec{v} ; \quad \overleftrightarrow{D}=\nu_{u}(k T / m) \stackrel{\leftrightarrow}{I}
$$

These coefficients are functionals of $f$ through the temperature and density so that Eq. (94) is a nonlinear operator, as is the case for the more exact operator of Eq. (11). However, in the following these will be assumed to be simply the temperature and density of $f_{0}$ so that $\mathrm{Cf}$ is linear. In the $\mathrm{v}, \chi$ coordinate system, the above operator becomes:

$$
C^{\prime} f=\frac{1}{2} \frac{\partial^{2} f}{\partial c^{2}}+\left(c+\frac{1}{c}\right) \frac{\partial f}{\partial c}+3 f+\frac{1}{2 c^{2} \sin x} \frac{\partial}{\partial x}\left(\sin x \frac{\partial f}{\partial x}\right)
$$

where $\mathrm{c}=\mathrm{v} / \alpha$. This operator is $\mathrm{known}^{32}$ to conserve particle number and energy, and to relax to a Maxwellian.

In Fig. 11, results for transport coefficients obtained with the functional expansion and ADI solution methods are plotted vs e $\phi_{\mathrm{o}} / \mathrm{kT}$ for $\nu_{0} / \Omega_{0}=1$ and positive fields. As was mentioned earlier, the functions used in the first method are exact eigenfunctions of the operator given in Eq. (96) so that the collision operator matrix is diagonal. The ADI results are, in most cases, close to the functional representation. results with somewhat better agreement obtained using a $50 \times 50$ grid than a $25 \times 25$ grid.

In Fig. 12, results are presented for $D_{n}^{\prime}$ both with inwardly and outwardly pointing ambipolar fields over the range $-1<\mathrm{e} \phi_{\mathrm{o}} / \mathrm{kT}<1$ and again for $\nu_{0} / s_{0}=1$. In this case, $s_{0}$ has been defined slight Ly differently than for the results with $e_{0} / k T>0$; it includes only drifts related to $\vec{\nabla} \mathrm{B}$. 
ORNL;'DWG/FED-7814

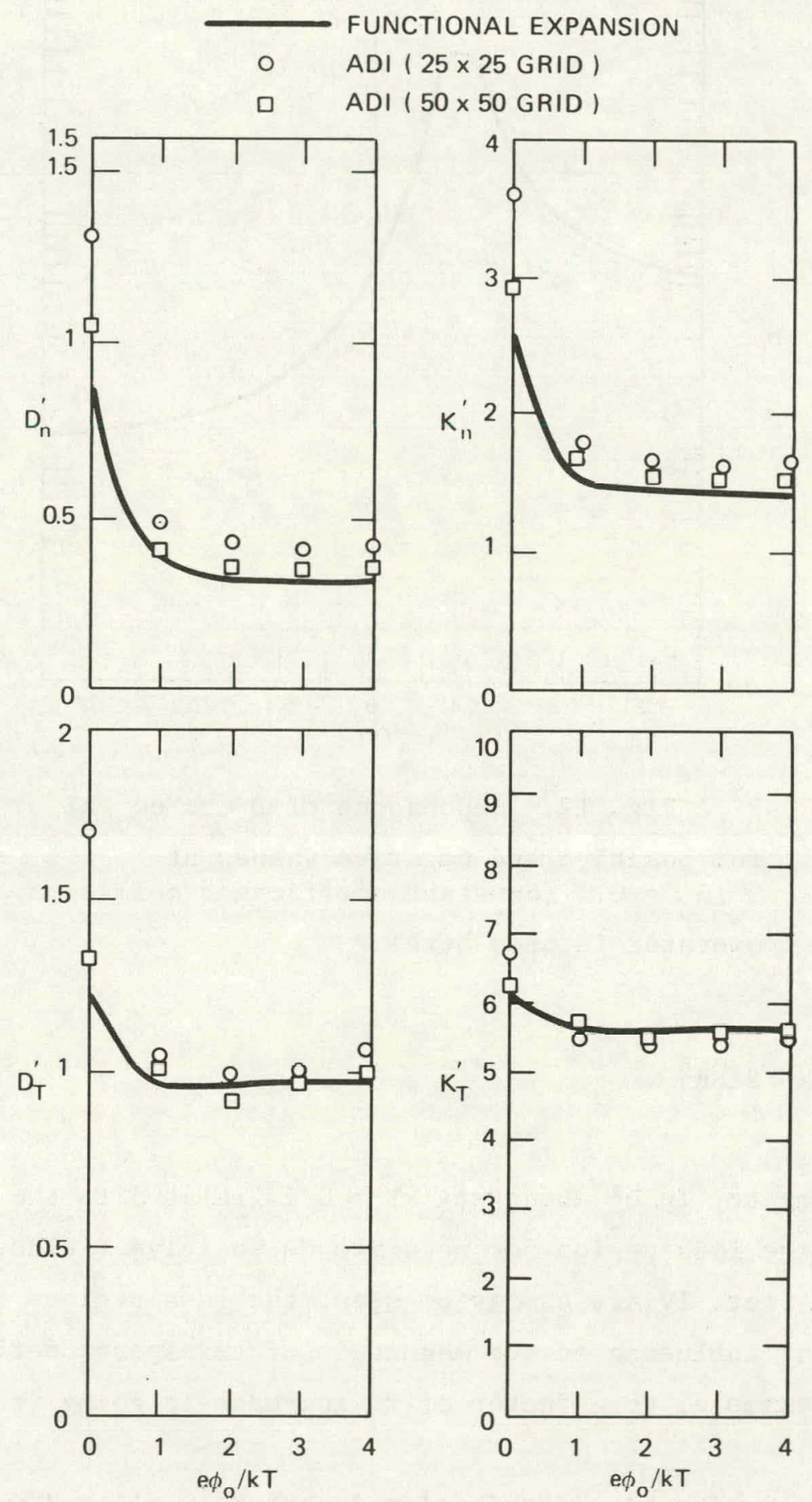

Fig. 11. Comparison between functional expansion and ADI finite difference solutions for the four transport coefficients vs e $\phi_{o} / \mathrm{kT}$ and at $\nu_{0} / \Omega_{0}=1$. These results are obtained with the constant coefficient collision operator. 32 


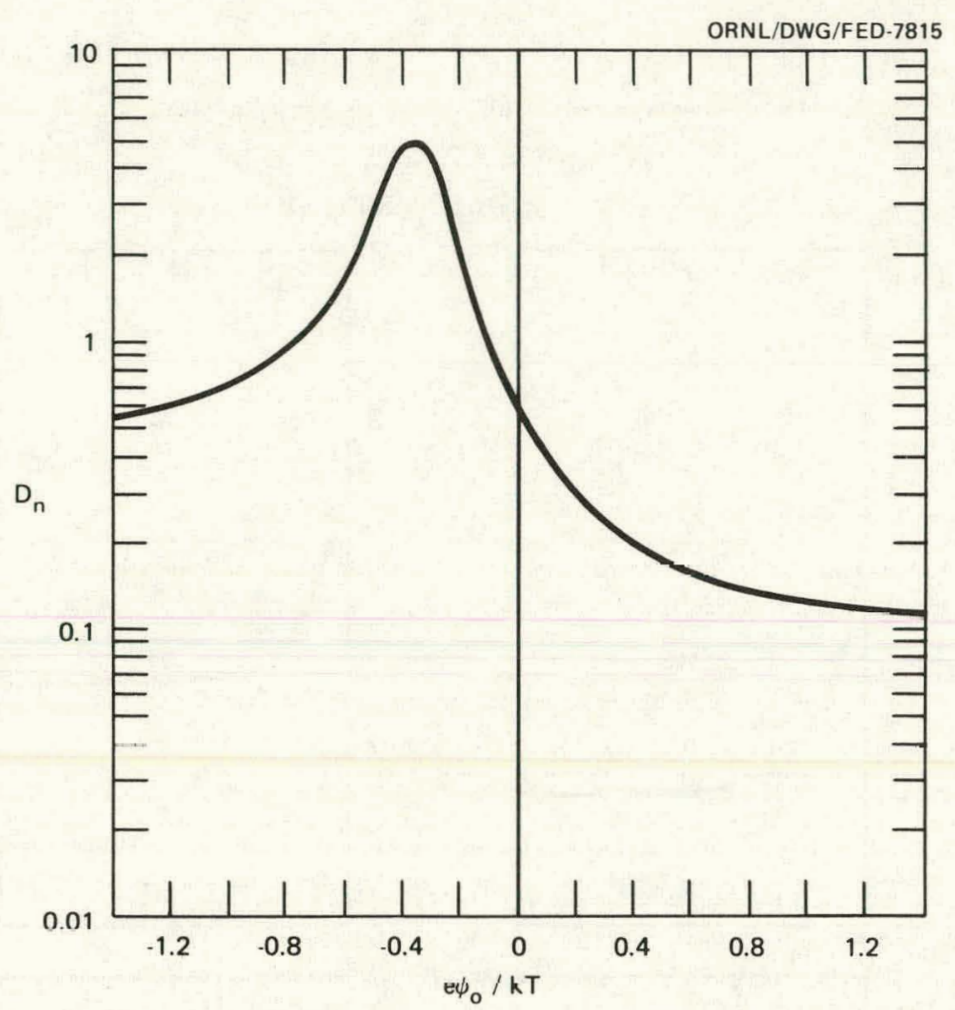

Fig. 12. Dependence of $\mathrm{D}_{\mathrm{n}}$ on $\mathrm{e} \phi_{\mathrm{o}} / \mathrm{kT}$

for positive and negative values at $\nu_{u} / \Omega_{u}=0.1$ (constant coefficient collision operator is used here).

$\Omega_{0}=\mathrm{N}^{2} \mathrm{kT} / 2 \mathrm{eBR} R^{2}$

The asymmetry in $D_{n}^{\prime}$ about e $\phi_{0} / k T=0$ is related to the different location of the loss region for negative vs positive fields, as has been discussed in Sect. IV.A. As may be seen, the loss regions can have a very important influence on the magnitude of transport coefficients, leading for example, to a factor of 20 increase in going from $\mathrm{e} \psi_{\mathrm{o}} / \mathrm{kT}-$ +0.4 to -0.4 .

In Fig. 13, the $f_{\text {sn }}^{\prime}$ distribution function is plotted vs velocity and $\zeta(=\cos x)$ for e $\phi_{0} / k T=-0.4$ and $\nu_{0} / \Omega_{0}=1$. The location of the peak in this distribution is closely related to the $\Omega=0$ contour, indicating its importance in determining the transport rates. 

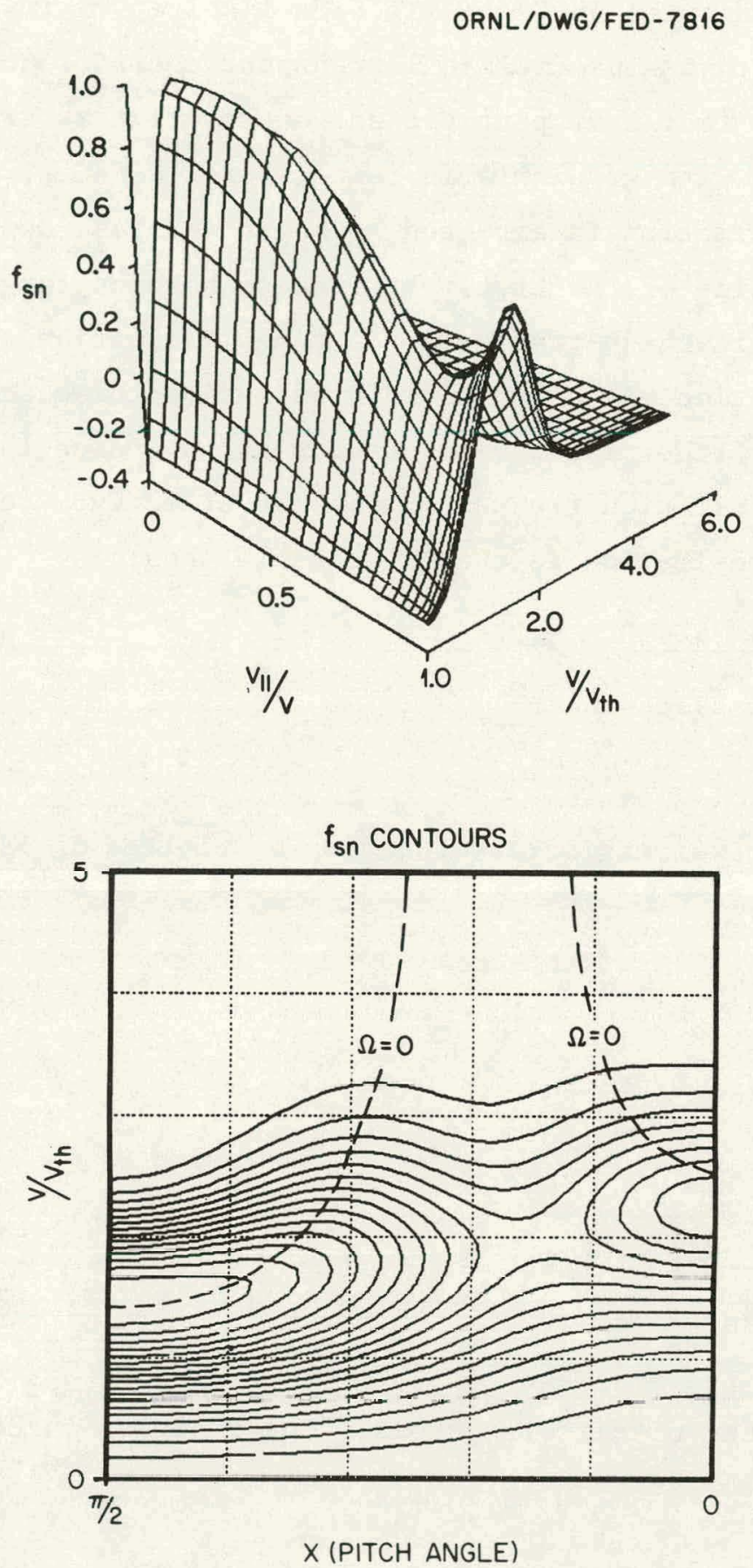

Fig. 13. $f_{s n}^{\prime}$ distribution function vs $\zeta$ and $v / v_{\text {th }}$ for e $\phi_{\mathrm{o}} / \mathrm{kT}=-0.4$ and $\nu_{0} / \Omega_{0}=0.1$. 


\section{Range of Validity for Local Approximation}

As was indicated in Sect. III.B, a local approximation in radius is inherent in deriving neoclassical transport coefficients. This is related to the fact that particle and energy fluxes have been assumed to scale linearly with gradients in temperature, density, and potential. Such an approximation is expected to begin to break down when the average step size of the transport process becomes large relative to scale lengths in these gradients. A rough indication of the validity of this approximation may be obtained by assuming these scale lengths are of the order of the plasma minor radius and by using the fact that $D_{n}$ is equal to the collision frequency times an effective step size squared. The local approximation is then reasonable when:

$$
\Delta x=\text { step size }=\sqrt{\frac{D_{n}}{v_{0}}}<a
$$

In terms of the dimensionless function $D_{n}^{\prime}$ plotted in Fig. 9, this becomes:

$$
\operatorname{lis}_{n} v_{n} a^{2}>v_{n}^{2} D_{n}^{\prime}
$$

or

$$
\mathrm{D}_{\mathrm{n}}^{\prime}<2\left(\frac{\Omega_{\mathrm{o}}}{\mathrm{v}_{\mathrm{o}}}\right)^{2}\left(\frac{\nu_{\mathrm{o}}}{\Omega_{\mathrm{o}}}\right) \mathrm{a}^{2}<\frac{\mathrm{N}^{4}}{8}\left(\frac{\mathrm{a}}{\mathrm{R}}\right)^{2}\left(\frac{\nu_{\mathrm{o}}}{\Omega_{\mathrm{o}}}\right)\left[1+\frac{4}{\mathrm{~N}^{2}}\left(\frac{\mathrm{R}}{\mathrm{a}}\right)^{2} \frac{\mathrm{e} \phi_{\mathrm{o}}}{\mathrm{kT}}\right],
$$

where $N=$ number of mirror sectors. For example, in EBT-I the above inequality becomes:

$$
D_{n}^{\prime}<415\left(\frac{\nu_{0}}{\Omega_{0}}\right)\left(1+0.7 \frac{\mathrm{e}_{0}}{\mathrm{kT}}\right)
$$


Based on the values of $D_{n}^{\prime}$ plotted in Fig. 9, it would appear that this is easy to satisfy for parameters characteristic of the present device $\left(\nu_{i} / \Omega_{i} \simeq 0.1, \nu_{e} / \Omega_{e} \simeq 1\right.$, e $\left.\phi_{0} / k \mathrm{kT} \simeq 1\right)$. In Fig. 14, a plot is given of $D_{n}^{\prime}$ vs e $\phi_{0} / k T$ and $\nu_{0} / \Omega_{0}$ along with Eq. (100) for e $\phi_{0} / k T=0$ (worst case). Except at very low values of $\nu_{0} / \Omega_{0}$ (not typical of the present experiment), it appears that a local approximation is justified. In the collisionless, large field limit (where $D_{n}^{\prime}=\nu_{0} / 12 \Omega_{0}$ ), Eq. (99) reduces to:

$$
\frac{\mathrm{e} \phi_{\mathrm{o}}}{\mathrm{kT}}>\frac{1}{6 \mathrm{~N}^{2}} \text {, }
$$

which should be readily satisfied.

Since we have assumed in this discussion that gradient scale lengths are of the order of the plasma minor radius, a slightly more stringent inequality may result in certain regions (such as near the plasma edge) where gradients can be much steeper than this. However, the neoclassical transport coefficients presented in this paper are not expected to be applicable in such regions due to neglect of the finite beta rings and the unstable cold plasma which exists just outside the rings. 
ORNL/DWG/FED-7817R

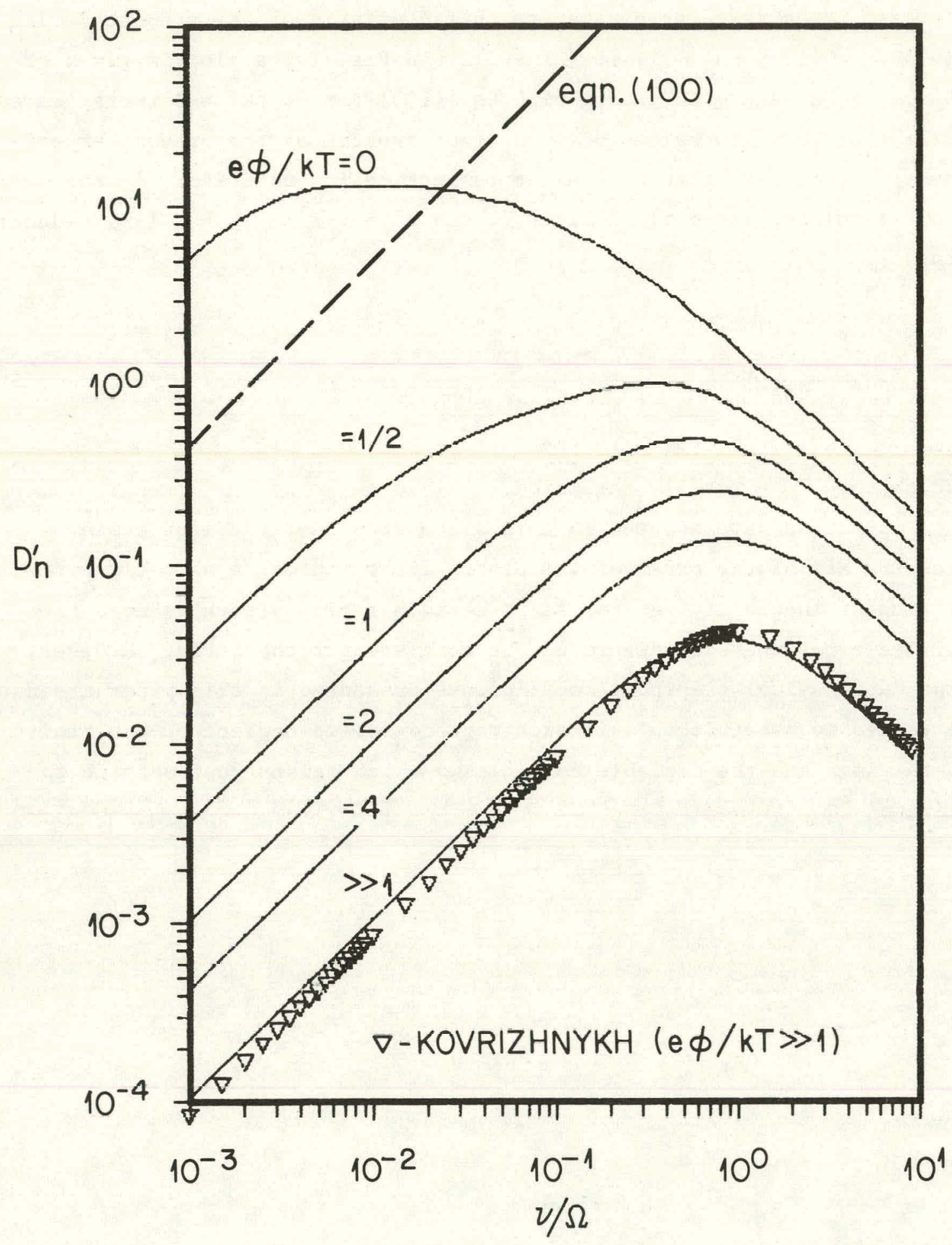

Fig. 14. Upper limit on $D_{n}^{\prime}$ for validity of the local approximation as indicated by Eq. (100). 


\section{PARTICLE AND ENERGY LIFETIME ESTIMATES}

The basic relevance of the results presented in this paper to the modeling of bumpy torus experiments is their influence on the plasma particle and energy lifetimes. In this section we shall make some simple estimates of these quantities for the EBT-I device. These will be based on assumed forms for the plasma density and temperature profiles and as such can indicate only the general scalings of these lifetimes with density, temperature, $\nu_{0} / \Omega_{0}$, and $\mathrm{e} \phi_{0} / \mathrm{kT}$. More refined estimates depend on better knowledge of the profiles; this can come either from one-dimensional fluid calculations ${ }^{23}$ or from experimental measurements.

The energy and particle balance equations are of the following form.

$$
\begin{aligned}
& \frac{\partial \mathrm{n}}{\partial t}+\frac{1}{r} \frac{\partial}{\partial r}\left(\mathrm{rS}_{r}\right)=S_{n}=\text { particle source. } \\
& \frac{\partial}{\partial t}\left(\frac{3}{2} \mathrm{nkT}\right)+\frac{1}{r} \frac{\partial}{\partial r}\left(r Q_{r}\right)=S_{E}=\text { energy source. }
\end{aligned}
$$

In sleady state the clme derivatives are zero and

$$
\begin{aligned}
& S_{n}=\frac{n}{\tau_{p}}, \\
& S_{E}=\frac{3}{2} \mathrm{nkT} / \tau_{E} .
\end{aligned}
$$

The particle and energy fluxes have been given in Eqs. (40) and (41). A Bessel function profile is assumed for temperature and density. In this case, Eq. (102) reduces to the following. 


$$
\tau_{\mathrm{p}}^{-1}=\frac{\mathrm{v}_{\mathrm{o}}^{2}}{2 \Omega_{\mathrm{o}}}\left(\frac{2.405}{\mathrm{a}}\right)^{2}\left[\mathrm{D}_{\mathrm{n}}^{\prime}\left(1+\frac{\mathrm{e} \phi_{\mathrm{o}}}{\mathrm{kT}}\right)+\mathrm{D}_{\mathrm{T}}^{\prime}\right]
$$

and

$$
\tau_{\mathrm{E}}^{-1}=\frac{\mathrm{v}_{\mathrm{o}}^{2}}{2 \Omega_{\mathrm{o}}}\left(\frac{2.405}{\mathrm{a}}\right)^{2}\left[\mathrm{~K}_{\mathrm{n}}^{\prime}\left(1+\frac{\mathrm{e} \phi_{\mathrm{o}}}{\mathrm{kT}}\right)+\mathrm{K}_{\mathrm{T}}^{\prime}\right],
$$

where $a=$ plasma minor radius; the other quantitiess were defined in the previous section. For EBT-I we assume the parameters: $\mathrm{B}_{\text {avg }}=0.9 \mathrm{I}$ (24 coils), $\mathrm{a}=10 \mathrm{~cm}$, and $\mathrm{R}=150 \mathrm{~cm}$. Then $\mathrm{Eq} \cdot$ (103) becomes:

$$
\tau_{\mathrm{p}}=\frac{-5.5\left(\frac{1 \mathrm{keV}}{\mathrm{kT}}\right)\left(1+0.7 \frac{\mathrm{e} \phi_{\mathrm{o}}}{\mathrm{kT}}\right)}{\mathrm{D}_{\mathrm{n}}^{\prime}\left(1+\frac{\mathrm{e} \phi_{\mathrm{o}}}{\mathrm{kT}}\right)+\mathrm{D}_{\mathrm{T}}^{\prime}} \quad \text { (in msec) }
$$

and

$$
\tau_{E}=\frac{8.2\left(\frac{1 \mathrm{keV}}{\mathrm{l.T}}\right)\left(1+1.1 \frac{\mathrm{e} \phi_{\mathrm{O}}}{\mathrm{k}^{\prime} !}\right)}{\mathrm{K}_{\mathrm{n}}^{\prime}\left(1+\frac{\mathrm{e} \phi_{\mathrm{o}}}{\mathrm{kT}}\right)+\mathrm{K}_{\mathrm{T}}^{\prime}} \quad \text { (1.n msec) }
$$

$D_{n}^{\prime}, D_{T}^{\prime}, K_{n}^{\prime}, K_{T}^{\prime}$ are the functions plotted in F1g. 9 and depind on $\nu_{0} / \Omega_{0}$ and $e \phi_{0} / \mathrm{kT}$. For EB'l-1 parameters, the quantity $\nu_{0} / s_{0}$ may be wrILlew as:

$$
\frac{\nu_{0}}{\Omega_{0}}=C_{0}\left(1+0.7 \frac{\mathrm{e} \phi_{0}}{\mathrm{kT}}\right)^{=1}\left(\frac{\mathrm{n}}{10^{12} \mathrm{~cm}^{-3}}\right)\left(\frac{\mathrm{kT}}{100 \mathrm{eV}}\right)^{-5 / 2},
$$


where

$$
\mathrm{C}_{\mathrm{o}}=\left\{\begin{array}{l}
0.078 \text { (ions) } \\
3.4 \text { (electrons) }
\end{array}\right.
$$

The particle and energy lifetimes, $\tau_{p}$ and $\tau_{E}$, given by Eq. (104) are plotted in Fig. $15 \mathrm{vs} \nu_{0} / \Omega_{0}$ and $e \phi_{0} / \mathrm{kT}$. The lowest case here is for e $\phi_{\mathrm{o}} / \mathrm{kT}=0$ with the next successive higher lines representing $\mathrm{e}_{\mathrm{o}} / \mathrm{kT}=$ $0.5,1,2$, and 4 .

Figure 15 indicates that for the parameter range considered, $\tau_{p}$ always exceeds $\tau_{E}$ by a factor in the range of 3 to 10 . Also, for values of collisionality and potential which are typical of the EBT experiment $(\nu / \Omega \simeq 0.1$ for ions, e $\phi / \mathrm{kT} \simeq 1)$, we note that $\tau_{\mathrm{p}} \simeq 17 \mathrm{msec}$ and $\tau_{\mathrm{E}} \simeq 5 \mathrm{msec}$.

ORNL / DWG /FED-7818R
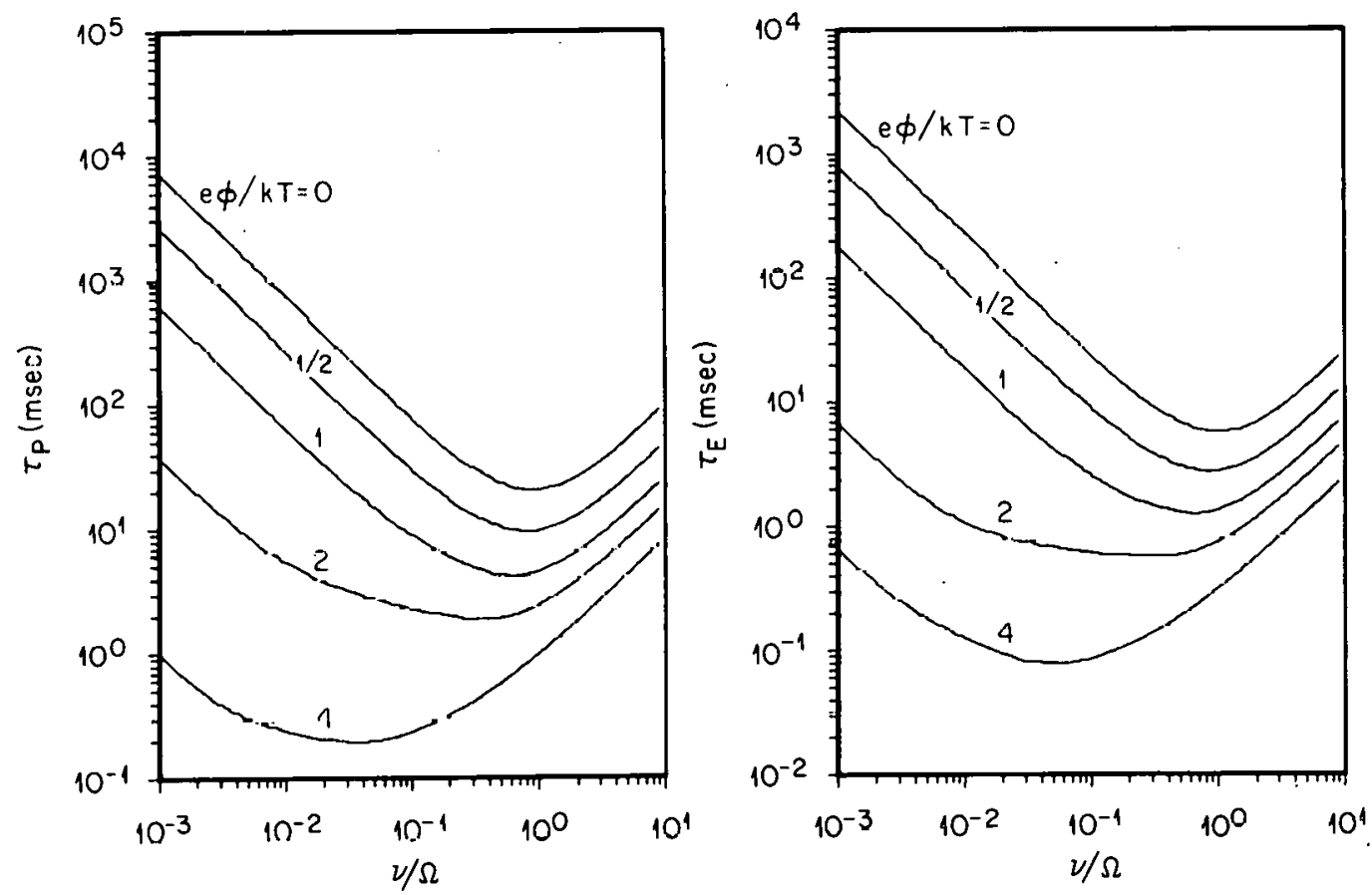

Fig. 15. Neoclassical particle and energy lifetimes (msec) as a function of collisionality $\left(\nu_{0} / \Omega_{0}\right)$ and ambipolar field $\left(\mathrm{e} \phi_{0} / \mathrm{kT}\right)$ for EBT-I parameters. 
THIS PAGE

\section{WAS INTENTIONALLY \\ LEFT BLANK}




\section{VII: SUMMARY AND CONCLUSIONS}

In the preceding sections, the basic theory and methods have been outlined for the calculation of neoclassical transport coefficients in a bumpy torus. Also, a number of specific results were presented which apply to the EBT-I device. The transport coefficients which we have obtained indicate a similar scaling with respect to collisionality - $(\nu / \Omega)$ as those given earlier by Kovrizhnykh.1,7 However, our results represent an extension to the earlier work with respect to the dependence on the ambipolar electric field. The methods and results indicated here are applicable for arbitrary values of the ambipolar field whereas before, only the large field limit had been considered. In the case of finite ambipolar fields $\left(e \phi_{o} / k T \simeq 1\right)$, it is found that the presence of regions in velocity space where particle orbits are not well centered. can substantially influence the transport coefficients and result in a stronger dependence on the ambipolar potential than is the case in the large field limit. Also, transport coefficients near e $\phi_{0} / \mathrm{kT}=0$ exhibit a marked dependence on the direction of the ambipolar field, indicating a considerable enhancement for slightly negative values of $e \phi_{o} / k T$ (inwardly pointing fields for positive particles). This behavior of the transport coefficients is of importance in determining the direction and size of the self-consistently calculated ambipolar electric field.

Due to the higher degree of structure which is present in the ion distribution function for inwardly pointing ambipolar fields vs outwardly pointing fields, it has been necessary to use differing methods in solving the kinetic equation for each case. For positive fields, a functional representation of the distribution in terms of Legendre and generalized Laguerre polynomials was used and seems to be adequate down to $e \phi_{0} / k T=0$. For negative ambipolar fields, the functional expansion generally tails to converge and a numerical tinite ditference (ADI) method was employed.

The analysis presented here is based on a number of approximations. The most fundamental is probably the local approximation; however, the range of validity for this has been examined in Sect. V.C and appears to 
be satisfactory for most plasma parameters of experimental interest. In addition, various simplifications are inherent in the particular results presented here. For example, the collision operator and $v_{y}$ drift have not been bounce averaged in the present results. Such averages have been given in Sects. II and IV and will be incorporated in future work on the problem. Also, it is expected that the particle orbit model will be upgraded in the near future to include the influence of finite beta on the equilibrium magnetic fields. Finally, the effects of $\mathrm{E}_{\theta}$ components in the ambipolar field on transport are of interest and are presently under consideration using simplified model collision operators. 
APPENDIX A - PRECESSIONAL DRIFT FREQUENCY MATRIX ELEMENTS

We shall consider a simple fit to $\Omega^{\prime}$ of the form given below:

$$
\frac{\Omega^{\prime}}{c^{2}}=a+b \zeta^{2}+d \zeta^{4}
$$

where $a, b$, and $d$ are constants and $\zeta=\cos x$. A typical fit for the central plasma region (assuming low beta) was given in Eq. (70). The matrix elements of interest were given in $\mathrm{Eq} .(84)$ as:

$$
\begin{array}{r}
\left\langle\mathrm{n}^{\prime} \ell^{\prime}\left|\Omega^{\prime}\right| \mathrm{n} \ell\right\rangle=\frac{4}{\pi^{1 / 2}} \int_{0}^{\infty} \mathrm{c}^{2} \mathrm{dc} \int_{0}^{1} \mathrm{~d} \zeta \mathrm{e}^{-c^{2}} \mathrm{R}_{\mathrm{n}^{\prime} \ell^{\prime}}(\mathrm{c}) \mathrm{Y}_{\ell^{\prime} \mathrm{o}}(\zeta) \\
\quad \times \Omega^{\prime}(\mathrm{c}, \zeta) \mathrm{R}_{\mathrm{n} \ell}(\mathrm{c}) \mathrm{Y}_{\ell \mathrm{o}}(\zeta) .
\end{array}
$$

The functions $R_{n \ell}(c)$ and $Y_{\ell o}(\zeta)$ are as given below: ${ }^{33}$

$$
\begin{aligned}
& \mathrm{R}_{\mathrm{n} \ell}(\mathrm{c})= {\left[2 \pi^{3 / 2} \Gamma\left(\frac{\mathrm{n}}{2}-\frac{\ell}{2}+1\right) \Gamma\left(\frac{\mathrm{n}}{2}+\frac{\ell}{2}+\frac{3}{2}\right)\right]^{1 / 2} } \\
& \times \times \sum_{\mathrm{m}=0}^{(\mathrm{n}-\ell) / 2}(-1)^{\mathrm{m}}\left[\Gamma\left(\frac{\mathrm{n}}{2}-\frac{\ell}{2}-\mathrm{m}+1\right)\right. \\
&\left.\times \Gamma\left(\mathrm{m}+\ell+\frac{3}{2}\right) \Gamma(\mathrm{m}+1)\right]^{-1} c^{2 m+\ell} . \\
& Y_{\ell 0}(\zeta)=\sqrt{\frac{2 \ell+1}{4 \pi}} \mathrm{P}_{\ell}(\zeta),
\end{aligned}
$$

where $\Gamma$ = the gamma function. Substituting Eq. (A.1) into Eq. (A.2) results in the following expression:

$$
\left\langle n^{\prime} \ell^{\prime}\left|\Omega^{\prime}\right| n \ell\right\rangle=\frac{\sqrt{2 \ell+1} \sqrt{2 \ell^{\prime}+1}}{\pi^{3 / 2}} I_{c^{3}} I_{\zeta} \text {, }
$$


where

$$
\begin{aligned}
I_{c}= & \int_{0}^{\infty} c^{4} d c e^{-c^{2}} R_{n} \cdot \ell^{\prime} R_{n \ell}, \\
I_{\zeta}= & \int_{0}^{1} d \zeta\left(a+b \zeta^{2}+d \zeta^{4}\right) P_{\ell} P_{\ell}, \cdot
\end{aligned}
$$

The pitch-angle integral, $I_{\zeta}$, may be evaluated by using the two identities given below.

$$
\begin{aligned}
& (2 \Omega+1) \zeta L_{\ell}(\zeta)=(U+1) P_{\ell+1}(l)+\ell \cdot P_{\ell-1}(\zeta) . \\
& \int_{0}^{1} d \zeta P_{\ell}(\zeta) P_{\ell^{\prime}}(\zeta)=\frac{1}{2 \ell+1} \delta \ell^{\prime}(\ell \text { even }) .
\end{aligned}
$$

For example, the second term in $I_{\zeta}$ is integrated by multiplying Eq. (A.6) by $\zeta$ and then applying Eq. (A.6) to the right-hand side of the resulting equation. This gives the following.

$$
\begin{aligned}
& \zeta^{2} \mathrm{P}_{\ell} \mathrm{P}_{\ell}=(2 \ell+1)^{-1}\left(2 \ell^{\prime}+1\right)^{-1}\left[(\ell+1)\left(\ell^{\prime}+1\right) \mathrm{P}_{\ell+1} \mathrm{P}_{\ell^{\prime}+1}\right. \\
& \left.\quad+\ell\left(\ell^{\prime}+1\right) \mathrm{P}_{\ell \ell^{\prime}+1} \mathrm{P}_{\ell-1}+\ell^{\prime}(\ell+1) \mathrm{P}_{\ell-1} \mathrm{P}_{\ell+1}+\ell \ell^{\prime} \mathrm{P}_{\ell \ell^{\prime}-1} \mathrm{P}_{\ell-1}\right],
\end{aligned}
$$

which may be integrated using Eq. (A.7) to obtain:

$$
\begin{aligned}
& \int_{0}^{1} \mathrm{~d} \zeta \zeta^{2} \mathrm{P}_{\ell^{2}} \mathrm{P}_{\ell^{\prime}}=\left(2 \ell^{\prime}+1\right)^{-1}(2 \ell+1)^{-1}\left[\frac{(\ell+1)\left(\ell^{\prime}+1\right)}{2 \ell^{\prime}+3} \delta_{\ell \ell^{\prime}}\right. \\
& \left.\quad+\frac{\ell \ell^{\prime}}{2 \ell^{\prime}-1} \delta_{\ell \ell^{\prime}}+\frac{\ell\left(\ell^{\prime}+1\right)}{2 \ell^{\prime}+3} \delta_{\ell^{\prime}+2, \ell}+\frac{\ell^{\prime}(\ell+1)}{2 \ell^{\prime}-1} \delta_{\ell^{\prime}-2, \ell}\right] .
\end{aligned}
$$


The first term in $I_{\zeta}$ is just the integral of Eq. (A.7). The last term of $I_{\zeta}, \int_{0}^{1} d \zeta \zeta^{4} P_{\ell} P_{\ell}$, has also been done in the same manner as the second term, i.e., by repeated application of identities (A.6) and (A.7), however it will not be indicated here as it is rather lengthy. The velocity integral, $I_{c}$, is of the following form:

$$
I_{c}=\int_{0}^{\infty} c^{4} d c e^{-c^{2}} \sum_{m=0}^{(n-\ell) / 2} a_{m} c^{2 m+\ell} \sum_{m^{\prime}=0}^{\left(n^{\prime}-\ell^{\prime}\right) / 2} a_{m^{\prime}} \cdot c^{2 m^{\prime}+\ell^{\prime}},
$$

where the coefficient $a_{m}$ is obtained from Eq. (A.3).

$$
a_{m}=\frac{(-1)^{m}\left[2 \pi^{3 / 2} \Gamma\left(\frac{\mathrm{n}}{2}-\frac{\ell}{2}+1\right) \Gamma\left(\frac{\mathrm{n}}{2}+\frac{\ell}{2}+\frac{3}{2}\right)\right]^{1 / 2}}{\Gamma\left(\frac{\mathrm{n}}{2}-\frac{\ell}{2}-\mathrm{m}+1\right) \Gamma\left(\mathrm{m}+\ell+\frac{3}{2}\right) \Gamma(\mathrm{m}+1)} .
$$

Multiplying the two sums in Eq. (A.10) leads to the following result for the velocity integrand:

$$
I_{c}=\frac{1}{2} \sum_{\substack{m=0 \\(m \text { even })}}^{n+n^{\prime}} A_{2 m^{\prime}} \Gamma\left(\frac{m}{2}+\frac{5}{2}\right)
$$

where the $A_{m}$ 's are the product coefficients of the two sums in Eq. (A.10).

$$
\sum_{m=0}^{(n-\ell) / 2} a_{m} c^{2 m i l \ell} \sum_{m^{\prime}=0}^{\left(n^{\prime}-\ell^{\prime}\right) / 2} a_{m}, c^{2 m^{\prime}+\ell^{\prime}}=\sum_{\substack{m=0 \\(m \text { even })}}^{n^{\prime}+n} a_{m} c^{m},
$$


that is,

$$
\begin{aligned}
& A_{0}=a_{0}^{2} \\
& A_{2}=2 a_{0} a_{2} \\
& A_{4}=a_{2}^{2}+2 a_{0} a_{4} \\
& \vdots \\
& A_{n+n^{\prime}}=a_{n^{\prime} n^{\prime}} .
\end{aligned}
$$

A subroutine was written to evaluate the $A_{m}$ coetticients for arbitrary values of $\mathrm{n}, \ell, \dot{n}^{\prime}$, and $\ell^{\prime}$. Th1s 1 s then called by another: program which performs the sum in Eq. (A.12) to obtain the velocity integral $I_{c}$. This is then multiplied by the pitch-angle integral, $I_{\zeta}$, and the factor in front of Eq. (A.5) to obtaln the desired matrix elements. $I_{\zeta}$ is evaluated by a subroutine which sums expressions of the type given in Eq. (A.9) for arbitrary values of $n, l, n^{\prime}$, and $\ell^{\prime}$. 


\section{APPENDIX B - LANDAU COLLISION OPERATOR MATRIX ELEMENTS}

The collision operator which has been used here is that given by Landau: 34

$$
\left(\frac{\partial f a}{\partial t}\right)_{\operatorname{col1}}=\sum_{b} \Gamma_{a b} \frac{\partial}{\partial v_{i}} \int d^{3} v^{\prime}\left(F_{b}^{\prime} \frac{\partial F_{a}}{\partial v_{j}}-\frac{m_{a}}{m_{b}} F_{a} \frac{\partial F_{b}^{\prime}}{\partial v_{j}^{\prime}}\right) \frac{\partial^{2}}{\partial v_{i} \partial v_{j}}\left|\vec{v}-\vec{v}^{\prime}\right|,
$$

where subscripts $a$ and $b$ denote species; $F_{b}^{\prime}=F\left(\vec{v}^{\prime}\right)$, and

$$
\Gamma_{\mathrm{ab}}=\frac{2 \pi^{2} \mathrm{e}_{\mathrm{a}}^{2} \mathrm{e}_{\mathrm{b}}^{2}}{\mathrm{~m}_{\mathrm{a}}^{2}} \ln \Lambda_{\mathrm{ab}}
$$

Since $m_{e} \ll m_{i}$, the ions will behave like a single component gas and it is sufficient to consider only ion-ion collisions. In the following, we shall drop the subscripts $a$ and $b$ and linearize the collision operator in the following manner.

$$
\mathrm{f}=\mathrm{f}_{0}(1+\phi)=\mathrm{f}_{0}+\mathrm{f}_{1} \text {, }
$$

where

$$
\begin{aligned}
& f_{0}=n\left(\frac{m}{2 \pi k T}\right)^{3 / 2} e^{-c^{2},} \\
& f_{i}=f_{s} \sin \theta+f_{c} \cos \theta,
\end{aligned}
$$

and

$$
\vec{c}=\sqrt{m / 2 k^{\prime} I^{\prime}} \vec{v} \text {. }
$$


The linearized collision operator is as given below:

$$
\begin{aligned}
& C(f, f)=\Gamma \frac{\partial}{\partial v_{i}} \int d^{3} v^{\prime} \frac{\partial^{2}}{\partial v_{i} \partial v_{j}}\left|\vec{v}-\vec{v}^{\prime}\right|\left[\left(f_{0}^{\prime} \frac{\partial f_{o}}{\partial v_{j}}-f_{0} \frac{\partial F_{0}^{\prime}}{\partial v_{j}^{\prime}}\right)\right. \\
& \left.\quad+\left(f_{l}^{\prime} \frac{\partial f_{0}}{\partial v_{j}}-f_{l} \frac{\partial f_{o}^{\prime}}{\partial v_{j}^{\prime}}\right)+\left(f_{0} \frac{\partial f_{l}}{\partial v_{j}}-f_{0} \frac{\partial f_{l}^{\prime}}{\partial v_{j}^{\prime}}\right)+o\left(f_{l}^{2}\right)\right] .
\end{aligned}
$$

The first term in parentheses above is identically zero because $C\left(t_{0}, t_{0}\right)=U$. Since $\dot{t}_{1}=\dot{f}_{0} \phi$,

$$
\frac{\partial f_{1}}{\partial v_{j}}=f_{0} \frac{\partial \phi}{\partial v_{j}}-\frac{m}{2 k T} f_{o} v_{j}
$$

The collision term of Eq. (B.3) becomes:

$$
\begin{aligned}
& C(f, f)=\Gamma \frac{\partial}{\partial v_{j}} \int d^{3} v f_{o} f_{o}^{\prime}\left[\left(\frac{\partial \phi}{\partial v_{j}}-\frac{\partial \phi^{\prime}}{\partial v_{j}}\right)-\frac{m}{2 k T}\left(\phi+\phi^{\prime}\right)\left(v_{j}-v_{j}^{\prime}\right)\right] \\
& \ddot{x} \underset{\partial v_{i} \partial v_{j}}{\partial z}\left|\dot{v}-\dot{v}^{\prime}\right| .
\end{aligned}
$$

However, because

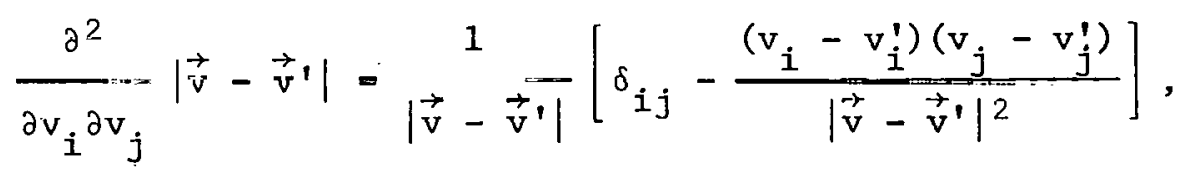

one finds that:

$$
\left(v_{j}-v_{j}^{\prime}\right) \frac{\partial^{2}}{\partial v_{i} \partial v_{j}}\left|\vec{v}-\vec{v}^{\prime}\right|=0
$$


The collision term then may be written as:

$$
\hat{c}_{1 \phi}=e^{c^{2}} \frac{\partial}{\partial c_{i}} \int \frac{d^{3} c^{\prime}}{\pi^{3 / 2}} e^{-\left(c^{2}+c^{\prime 2}\right)}\left(\frac{\partial \phi}{\partial c_{j}}-\frac{\partial \phi^{\prime}}{\partial c_{j}^{\prime}}\right) \frac{\partial^{2}}{\partial c_{i} \partial c_{j}}\left|\vec{c}-\vec{c}^{\prime}\right|,
$$

where we define

$$
\hat{C}_{1 \phi}=\frac{1}{\nu_{0}}\left(\frac{\partial \phi}{\partial t}\right)_{\operatorname{col1}}=\frac{1}{f_{0}} C(f, f),
$$

and

$$
\nu_{0}=\mathrm{n} \Gamma\left(\frac{\mathrm{m}}{2 \pi \mathrm{kT}}\right)^{3 / 2}
$$

It may be shown that this operator conserves particles, momentum, and energy, and is self-adjoint. Further, it is known ${ }^{35}$ that $\hat{C}_{1}$ satisfies the following relation:

$$
\hat{C}_{1} R(c) Y_{\ell m}(X, \beta)=S(c) Y_{\ell m}(X, \beta),
$$

where $R, S$ = arbitrary functions of velocity. In the following, we shall calculate the matrix elements $\left\langle n l\left|\hat{C}_{1}\right| n^{\prime} l^{\prime}\right\rangle$ using the functions given in Eq. (79). From the orthogonality of the $\mathrm{Y}_{\ell \mathrm{m}}$ 's and Eq. (B.9) one finds:

$$
<\mathrm{n} \ell\left|\hat{\mathrm{C}}_{1}\right| \mathrm{n}^{\prime} \ell^{\prime}>=0 \text { if } \quad \ell \neq \ell^{\prime}
$$

Thus it will only be necessary to calculate matrix elements for $\ell=\ell^{\prime}$ : These are given by: 


$$
\begin{aligned}
\left\langle\psi\left|\hat{C}_{1}\right| \phi\right\rangle=\pi^{-3} \int d^{3} c \psi \frac{\partial}{\partial c_{i}} & \int d^{3} c^{\prime} e^{-\left(c^{2}+c^{\prime 2}\right)} \\
& \times\left(\frac{\partial \phi}{\partial c_{j}}-\frac{\partial \phi^{\prime}}{\partial c_{j}^{\prime}}\right) \frac{\partial^{2}}{\partial c_{i} \partial c_{j}}\left|\vec{c}-\vec{c}^{\prime}\right|,
\end{aligned}
$$

where

$$
\psi(c, \zeta)=R_{n_{\ell}^{\prime}}(c) Y_{\ell o}(\zeta)
$$

and

$$
\phi(0, \zeta)=B_{n \ell}(c) Y_{\ell O}(\zeta)
$$

It is convenient to integrate this once by parts and write it in the following form:

$$
\left\langle\psi\left|\hat{C}_{1}\right| \phi\right\rangle=-\int \mathrm{d}^{3} \mathrm{c} \pi^{-3 / 2} \mathrm{e}^{-\mathrm{c}^{2}}\left(\frac{\partial \psi}{\partial c_{i}} \frac{\partial \phi}{\partial c_{j}} \dot{D}_{i j}-\frac{\partial \psi}{\partial c_{i}} \frac{\partial A}{\partial c_{i}}\right),
$$

where

$$
D_{i j}=\frac{\partial^{2}}{\partial c_{i} \partial c_{j}} \int d^{3} c^{\prime} \pi^{-3 / 2} e^{-c^{\prime 2}}\left|\vec{c}-\vec{c}^{\prime}\right|
$$

and

$$
A=\int d^{3} c \pi^{-3 / 2} e^{-c^{\prime 2}} \frac{\partial \phi^{\prime}}{\partial c_{j}^{\prime}} \frac{\partial}{\partial c_{i}}\left|\vec{c}-\vec{c}^{\prime}\right|
$$

'The integral $D_{i j}$ may be expressed in terms of error functions as follows: 


$$
D_{i j}=D_{\perp} \delta_{i j}+\left(D_{\|}-v_{1}\right) \frac{C_{i} c_{j}}{c^{2}}
$$

where

$$
\begin{aligned}
& D_{\perp}(c)=\frac{1}{c} \frac{\partial F}{\partial c}, \\
& D_{\|}(c)=\frac{\partial^{2} F}{\partial c^{2}},
\end{aligned}
$$

and

$$
F(c)=\frac{1}{2}\left[\left(2 c+\frac{1}{c}\right) \operatorname{erf}(c)+\frac{d}{d c} \operatorname{erf}(c)\right] \text {. }
$$

That is, $D_{\perp}$ (c) and $D_{\|}$(c) are given by:

$$
D_{\|}(c)=\frac{2}{\sqrt{\pi}}\left(\frac{1}{c^{3}} \int_{0}^{c} d c^{\prime} e^{-c^{\prime 2}}-\frac{1}{c^{2}} e^{-c^{2}}\right),
$$

and

$$
D_{1}(c)=\frac{2}{\sqrt{\pi}}\left[\left(\frac{1}{c}-\frac{1}{2 c^{3}}\right) \int_{0}^{c} d c^{\prime} e^{-c^{\prime 2}}+\frac{1}{2 c^{2}} e^{-c^{2}}\right] \text {. }
$$

The integrand of the first term in Eq. (B.11) may thus be written as:

$$
\begin{array}{r}
\frac{\partial \psi}{\partial c_{i}} \frac{\partial \phi}{\partial c_{j}} D_{i j}=D_{1} \frac{\partial \psi}{\partial c_{i}} \frac{\partial \phi}{\partial c_{i}}+\frac{\left(D_{\|}-D_{1}\right)}{c^{2}}\left(c_{i} \frac{\partial \psi}{\partial c_{i}}\right)\left(c_{j} \frac{\partial \psi}{\partial c_{j}}\right) \\
=D_{1} \vec{\nabla} \psi \cdot \vec{\nabla} \phi+\frac{\left(D_{\|}-D_{1}\right)}{c^{2}}(\vec{c} \cdot \vec{\nabla} \psi)(\vec{c} \cdot \vec{\nabla} \phi),
\end{array}
$$


where the gradient operators are understood to act in velocity space. Expressing Eq. (B.15) in spherical coordinates gives:

$$
\frac{\partial \psi}{\partial c_{i}} \frac{\partial \phi}{\partial c_{j}} D_{i j}=D_{\|} \frac{\partial \psi}{\partial c} \frac{\partial \phi}{\partial c}+\frac{D_{1}}{c^{2}}\left(\frac{\partial \psi}{\partial \chi} \frac{\partial \phi}{\partial \chi}+\frac{1}{\sin ^{2} x} \frac{\partial \psi}{\partial \beta} \frac{\partial \phi}{\partial \beta}\right),
$$

where $\beta=$ azimuthal angle (gyroangle) and $X=$ pitch angle. The first term of Eq. (B.11) then becomes:

$$
\int d^{3} \bar{c} \pi^{-3 / i} e^{-r^{2}} \frac{\partial \psi}{\partial c_{i}} \frac{\partial \phi}{\partial c_{j}} \tilde{u}_{1 j}=P+\dot{P}
$$

where

$$
P \equiv \int d^{3} c \pi^{-3 / 2} e^{-c^{2}} D_{\|} \frac{\partial \phi}{\partial c} \frac{\partial \psi}{\partial c}
$$

and

$$
Q \equiv \int d^{3} c \pi^{-3 / 2} e^{-c^{2}} \frac{D_{1}}{c^{2}}\left(\frac{\partial \psi}{\partial x} \frac{\partial \phi}{\partial x}+\frac{1}{s i n^{?} \times} \frac{\partial \psi}{\partial \beta} \frac{\partial \phi}{\partial \beta}\right)
$$

Integrating $Q$ by parts with respect to $X$ and $\beta$ gives the following:

$$
Q=-\int \frac{d^{3} c}{\pi^{3 / 2}} e^{-c^{2}} \psi \frac{D_{1}}{c^{2}}\left(\frac{1}{\sin \chi} \frac{\partial}{\partial \chi} \sin x \frac{\partial \phi}{\partial \chi}+\frac{1}{\sin ^{2} x} \frac{\partial^{2} \phi}{\partial \beta^{2}}\right) .
$$

However, since $\phi \sim \mathrm{Y}_{\ell \mathrm{m}}(X, \beta)$, the above reduces to:

$$
Q=\ell(\ell+1) \int d^{3} c \pi^{3 / 2} e^{-c^{2}} \frac{D_{1}}{c^{2}} \psi \phi
$$

Integrating $P$ and $Q$ over pitch angle and gyroangle results in the following expressions. 


$$
\begin{gathered}
P=\pi^{-3 / 2} \int_{0}^{\infty} \mathrm{d} c e^{-c^{2}} c^{2} D_{\|}(c) \frac{\partial R_{n} \ell \ell}{\partial c} \frac{\partial R_{n \ell}}{\partial c} . \\
Q=\pi^{-3 / 2} \ell(\ell+1) \int_{0}^{\infty} d c e^{-c^{2}} D_{1}(c) R_{n^{\prime} \ell}(c) R_{n \ell}(c) .
\end{gathered}
$$

We now return to the second term in Eq. (B.11) and simplify it first by an integration by parts:

$$
\begin{aligned}
T=-\int \frac{d^{3} c}{\pi^{3 / 2}} e^{-c^{2}} \frac{\partial \psi}{\partial c_{i}} \frac{\partial A}{\partial c_{i}}=\int \frac{d^{3} c}{\pi^{3 / 2}} e^{-c^{2}} \Lambda\left(\frac{\partial^{2} \psi}{\partial c_{i} \partial c_{i}}-2 c_{i} \frac{\partial \psi}{\partial c_{i}}\right) \\
=-2 n^{\prime} \int \frac{d^{3} c}{\pi^{3 / 2}} e^{-c^{2}} A \psi
\end{aligned}
$$

Here we have utilized the fact that the trial functions used to represent $\psi$ and $\phi$ are eigenfunctions of the Fokker-Planck operator with constant coefficients ${ }^{32}$ and satisfy the following equation.

$$
\frac{1}{2} \frac{\partial^{2} \psi_{n^{\prime} \ell}}{\partial c_{i} \partial c_{j}}-c_{j} \frac{\partial \psi_{n^{\prime} \ell}}{\partial c_{j}}=-n^{\prime} \psi_{n^{\prime} \ell} .
$$

The integral A may also be simplified in a similar manner by doing an integration by parts and using Eq. (B.22).

$$
\begin{aligned}
A & =\int \frac{\pi^{3 \vec{r}^{\prime}}}{\pi^{3 / 2}} e^{-r^{\prime 2}} \frac{\partial \phi^{\prime}}{\partial c_{j}^{\prime}} \frac{\partial}{\partial c_{j}}\left|\vec{c}-\vec{c}^{\prime}\right| \\
& =\int \frac{d^{3} c^{\prime}}{\pi^{3 / 2}} e^{-c^{\prime 2}}\left|\vec{c}=\vec{c}^{\prime}\right|\left(\frac{\partial^{2} \phi^{\prime}}{\partial c_{j}^{\prime} \partial c_{j}^{\prime}}-2 c_{j}^{\prime} \frac{\partial \phi^{\prime}}{\partial c_{j}^{\prime}}\right) \\
& =-2 n \int \frac{d^{3} c^{\prime}}{\pi^{3 / 2}} e^{-c^{\prime 2}}\left|\vec{c}-\vec{c}^{\prime}\right| \phi_{n l}^{\prime} .
\end{aligned}
$$


To further evaluate the integrals $A$ and $T$, it is necessary to expand $\left|\vec{c}-\vec{c}^{\prime}\right|$ in the spherical harmonics $Y_{\ell m}(X, \beta)$. Such an expression has been derived, for example, in Ref. 35 and is given below.

$$
\begin{aligned}
\left|\vec{c}-\vec{c}^{\prime}\right|=c_{>} \sum_{\ell=0}^{\infty} \sum_{\mathrm{m}=-\ell}^{\ell} \frac{4 \pi}{\left(4 \ell^{2}-1\right)}\left(\frac{c_{<}}{c_{>}}\right)^{\ell}\left[\left(\frac{2 \ell-1}{2 \ell+3}\right)\left(\frac{c_{<}}{c_{>}}\right)^{2}-I\right] \\
\\
\quad \times Y_{\ell \mathrm{III}}(x, \beta) \mathrm{Y}_{\ell \mathrm{III}}^{*}\left(x^{\prime}, \beta^{\prime}\right), \quad(B, 24)
\end{aligned}
$$

where $c_{>}$is the larger of $c$ and $c^{\prime}, c_{<}$is the smaller. Substituting Eq. (B.23) into Eq. (B.21) one has:

$$
\begin{aligned}
& T=4 \pi^{-3} n n^{\prime} \int d^{3} c \int d^{3} c^{\prime} e^{-\left(c^{2}+c^{\prime 2}\right)}\left|\vec{c}-\vec{c}^{\prime}\right| \\
& \quad \times R_{n^{\prime} \ell}(c) R_{n \ell^{\prime}}\left(c^{\prime}\right) Y_{\ell^{\prime} o}(X) Y_{\ell O}\left(X^{\prime}\right) .
\end{aligned}
$$

Substituting Eq. (B.24) into the above expression and using the orthogonality properties of the $\mathrm{Y}_{\ell m}$ functions give

$$
\begin{aligned}
& T=\frac{16 n n^{\prime}}{\pi^{2}\left(4 \ell^{2}-1\right)} \int_{0}^{\infty} c^{2} d c \int_{0}^{\infty} d c^{\prime} c^{\prime 2} e^{-\left(c^{2}+c^{\prime 2}\right)} R_{n^{\prime} \ell^{(c)}} R_{n \ell}\left(c^{\prime}\right) \\
& \times c_{>}\left(\frac{c_{s}}{c_{>}}\right)^{l}\left[\left(\frac{2 l-1}{2 l+3}\right)\left(\frac{c_{\dot{ }}}{c_{>}}\right)^{2}-1\right]
\end{aligned}
$$

To summarize, the collision operator matrix elements have been reduced to the following form.

$$
\left\langle\psi\left|\hat{\mathrm{C}}_{1}\right| \phi\right\rangle=-(P+Q+T) \delta_{\ell \ell^{\prime}},
$$


where $P, Q, T$ are the integrals given below:

$$
\begin{aligned}
& P=\pi^{-3 / 2} \int_{0}^{\infty} c^{2} d c e^{-c^{2}} D_{\|} \text {(c) } \frac{\partial R_{n^{\prime}} \ell}{\partial c} \frac{\partial R_{n \ell}}{\partial c} \text {; }
\end{aligned}
$$

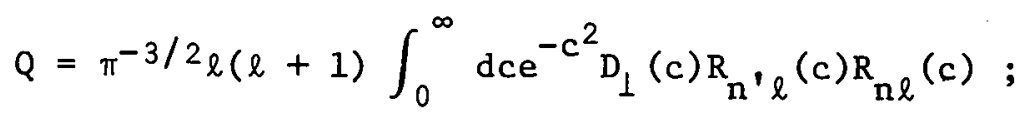

and

$$
\begin{aligned}
& T=\frac{16 n n^{\prime}}{\pi^{2}\left(4 l^{2}-1\right)} \int_{0}^{\infty} c^{2} d c \int_{0}^{\infty} d c^{\prime} c^{\prime 2} e^{-\left(c^{2}+c^{\prime 2}\right)} R_{n^{\prime} \ell}(c) R_{n \ell}\left(c^{\prime}\right) \\
& \times c_{>}\left(\frac{c_{<}}{c_{>}}\right)^{l}\left[\left(\frac{2 l-1}{2 l+3}\right)\left(\frac{c_{<}}{c_{>}}\right)^{2}-1\right]
\end{aligned}
$$

Here

$$
D_{\|}(c)=\frac{\dot{z}}{\sqrt{\pi}}\left(\frac{1}{c^{3}} \int_{0}^{r} \mathrm{dc} \mathrm{e}^{-\mathrm{c}^{\prime 2}}-\frac{1}{\mathrm{c}^{2}} \mathrm{e}^{-\mathrm{c}^{2}}\right),
$$

and

$$
D_{1}(c)=\frac{2}{\sqrt{\pi}}\left(\frac{1}{c}-\frac{1}{2 c^{3}} \int_{0}^{c} d c^{\prime} e^{-c^{\prime 2}}+\frac{1}{2 c^{2}} e^{-c^{2}}\right) .
$$

The function $R_{n \ell}(c)$ may be expressed in the following form: ${ }^{33}$ 


$$
\begin{aligned}
& \mathrm{R}_{\mathrm{n} \ell}(\mathrm{c})= {\left[2 \pi^{3 / 2} \Gamma\left(\frac{\mathrm{n}}{2}-\frac{\ell}{2}+1\right) \Gamma\left(\frac{\mathrm{n}}{2}+\frac{\ell}{2}+\frac{3}{2}\right)\right]^{1 / 2} } \\
& \times \sum_{\mathrm{m}=0}^{(\mathrm{n}-\ell) / 2}(-1)^{\mathrm{m}}\left[\Gamma\left(\frac{\mathrm{n}}{2}-\frac{\ell}{2}-\mathrm{m}+1\right) \Gamma(\mathrm{m}+1)\right. \\
&\left.\times \Gamma\left(\mathrm{m}+\ell+\frac{3}{2}\right)\right]^{-1} \mathrm{c}^{2 \mathrm{~m}+\ell} .
\end{aligned}
$$

When Eq. (B.29) is substituted into Eq. (B.28), all terms of P, Q, and T reduce to integrals of one of the following two forms.

$$
\begin{aligned}
& \mathrm{K}_{\mathrm{p}}=\int_{0}^{w} \mathrm{dce^{-c^{2 } }} \mathrm{c}^{\mathrm{p}} \text {. } \\
& J_{p q}=\int_{0}^{m} d c e^{-c^{2}} c^{p} \int_{0}^{c} d c^{\prime} e^{-c^{\prime 2}} c^{\prime q} .
\end{aligned}
$$

The $\mathrm{K}_{\mathrm{p}}$ integral may be done by a simple change of variables, resulting in:

$$
\mathrm{K}_{\mathrm{p}}=\left(\frac{1}{2}\right)^{(\mathrm{p}+3) / 2} \Gamma\left(\frac{\mathrm{p}}{2}+\frac{1}{2}\right)
$$

The $\mathrm{J}_{p q}$ 's may be evaluated by first making the transformation:

$$
\begin{aligned}
c & =r \cos \theta ; \\
c^{\prime} & =r \sin \theta,
\end{aligned}
$$

such that: 


$$
\begin{aligned}
J_{p q} & =\int_{0}^{\infty} r d r e^{-r^{2}} r^{p+q} \int_{0}^{\pi / 4} d \theta \cos ^{p} \theta \sin ^{q} \theta \\
& =\frac{1}{2} \Gamma\left(\frac{p}{2}+\frac{q}{2}+1\right) L_{p q},
\end{aligned}
$$

where

$$
\mathrm{L}_{\mathrm{pq}}=\int_{0}^{\pi / 4} \mathrm{~d} \theta \cos ^{\mathrm{P}} \theta \sin ^{\mathrm{q}} \theta
$$

Recurrence relations for the integrals $\mathrm{J}_{\mathrm{pq}}$ have been derived using integration by parts. These are given below:

$$
\begin{gathered}
J_{p, q+2}=\frac{q+1}{2} J_{p q}-\frac{1}{8}\left(\frac{1}{2}\right)^{(p+q) / 2} \Gamma\left(\frac{p+q}{2}+1\right) ; \\
J_{p+2, q}=\frac{p+1}{2} J_{p q}+\frac{1}{8}\left(\frac{1}{2}\right)^{(p+q) / 2} \Gamma\left(\frac{p+q}{2}+1\right) .
\end{gathered}
$$

All the required values of the $\mathrm{J}_{\mathrm{pq}}$ integrals may be obtained from these relations and from Eq. (B.34). One must start with the following elements of $\mathrm{J}_{\mathrm{pq}}$ :

$$
\begin{aligned}
& J_{00}=\pi / 8, \\
& J_{10}=\sqrt{2 \pi} / 8, \\
& J_{01}=\frac{\sqrt{\pi}}{4}\left(1-\frac{1}{2} \sqrt{2}\right),
\end{aligned}
$$

and

$$
\mathrm{J}_{11}=1 / 8
$$


In the code which calculates collision operator matrix elements, the $\mathrm{J}_{\mathrm{pq}}$ matrix is first calculated using Eqs. (B.37), (B.35), and (B.36). Next, the integrals $P, Q$, and $T$ are computed by adding up the appropriate terms involving the $\mathrm{J}_{\mathrm{pq}}$ and $\mathrm{K}_{\mathrm{p}}$ integrals (these relations are lengthy and have not been indicated here). These three integrals are then summed to obtain the collision operator matrix elements. 
APPENDIX C - VARIATIONAL PRINCIPLE FOR TRANSPORT COEFFICIENTS

In this appendix we shall derive a variational form for the density diffusion coefficient $D_{n}$. This coefficient was given in Eq. (88) as:

$$
-D_{n}=\frac{v_{0}^{2}}{2 v_{0}}\left\langle v_{y}^{\prime} \mid f_{s n}^{\prime}\right\rangle
$$

where $\left|f_{s n}^{\prime}\right\rangle$ was a solution of the following set of coupled equations.

$$
\begin{aligned}
& C^{\prime}\left|f_{\mathrm{sn}}^{\prime}\right\rangle+\lambda \Omega^{\prime}\left|\mathrm{f}_{\mathrm{cn}}^{\prime}\right\rangle=\left|\mathrm{v}_{\mathrm{y}}^{\prime}\right\rangle . \\
& C^{\prime}\left|\mathrm{f}_{\mathrm{cn}}^{\prime}\right\rangle-\Omega^{\prime}\left|\mathrm{f}_{\mathrm{sn}}^{\prime}\right\rangle=0 ; \quad \lambda \equiv\left(\Omega_{0} / \nu_{0}\right)^{2} .
\end{aligned}
$$

For simplicity in the following, primes will be dropped and the factor $v_{0}^{2} / 2 \nu_{0}$ in $D_{n}$ will be left off since it does not influence the proof. Recall that $\mathrm{C}^{\prime}$ is an Hermitian operator.

Using Eq. (C.2) in Eq. (C.1) for $\left\langle v_{y}^{\prime}\right|$ gives the following:

$$
-D_{n}=\lambda<f_{c n}|\Omega| f_{s n}>+\left\langle f_{s n}|c| f_{s n}>\right.
$$

Using Eq. (C.3), the identity given below may be derived:

$$
\left.\left\langle\mathrm{f}_{\mathrm{cn}}|\mathrm{C}| \mathrm{f}_{\mathrm{cn}^{\prime}}\right\rangle=<\mathrm{f}_{\mathrm{cn}}|\Omega| \mathrm{f}_{\mathrm{sn}}\right\rangle=\left\langle\mathrm{f}_{\mathrm{sn}}|\mathrm{s}| \mathrm{f}_{\mathrm{cn}}\right\rangle
$$

Dividing and multiplying Eq. (C.4) by $\left\langle f_{\mathrm{cn}}\left|r_{1}\right| f_{\mathrm{cn}}>\right.$ and using the abovo equation, one then obtains the following expression for $D_{n}$ :

$$
-D_{n}=\frac{\lambda<f_{s n}|\Omega| f_{c n}>^{2}+<f_{s n}|C| f_{s n}><f_{c n}|C| f_{c n}>}{<f_{c n}|C| f_{c n}>} .
$$


If we write $D_{n}$ as $D_{n}^{2} / D_{n}$ and use Eq. (C.1) for the numerator and Eq. (C.6) for the denominator, the form given below is obtained.

$$
-D_{n}=\frac{<v_{y}\left|f_{s n}>^{2}<f_{c n}\right| c \mid f_{c n}>}{\lambda<f_{s n}|\Omega| f_{c n}>^{2}+<f_{s n}|c| f_{s n}><f_{c n}|c| f_{c n}>} .
$$

From Eq. (C.7) it is now possible to demonstrate that the functions $f_{s n}$ and $f_{c n}$ which extremize $D_{n}$ are also. those which satisfy Eqs. (C.2) and (C.3). This is the same form as the variational principle given in Ref. 25. The proof is as followo.

First, $\left|f_{\text {on }}\right\rangle$ will be held fixed and $\left|f_{\mathrm{cn}}\right\rangle$ will be allowed to vary. It is then convenient to write Eq. (C.7) as follows:

$$
-D_{n}=\frac{<v_{y} \mid f_{s n}>^{2}}{\lambda \gamma+<f_{s n}|c| f_{s n}>}
$$

where

$$
\gamma=\frac{\left\langle f_{s n}|\Omega| f_{c n}{ }^{2}\right.}{\left\langle f_{c n}|c| f_{c n}>\right.}
$$

$-D_{n}$ i.s maximized by minimizing $\gamma$. This condition is given below:

$$
\begin{aligned}
& \delta \gamma<f_{\mathrm{LH}}|\mathrm{C}| \mathrm{f}_{\mathrm{eHi}}>+\gamma\left(<\mathrm{f}_{\mathrm{en}}|\mathrm{C}| \delta \mathrm{f}_{\mathrm{en}}>+\left\langle\delta \mathrm{f}_{\mathrm{on}}|\mathrm{C}| \mathrm{f}_{\mathrm{en}}>\right)\right. \\
& =2< \pm_{\mathrm{sn}}|s| \mathrm{f}_{\mathrm{cn}}><\mathrm{f}_{\mathrm{sn}}|s| \delta \mathrm{f}_{\mathrm{cn}} ; .
\end{aligned}
$$

Setting $\delta \gamma=0$ and using the Hermitian properties of $\Omega$ and $C$ results in:

$$
\left\langle\delta f_{c n}\right|\left(\gamma C f_{c n}-\left\langle f_{s n}|\Omega| f_{c n}>\Omega f_{s n}\right)\right\rangle=0,
$$


or, substituting Eq. (C.9) for $\gamma$, .

$$
\frac{<f_{s n}|\Omega| f_{c n}}{\left\langle f_{c n}|C| f_{s n}\right.}>C\left|f_{c n}>-\Omega\right| f_{s n}>=0 .
$$

Since the value of $\left|f_{c n}\right\rangle$ which satisfies Eq. (C.3) is exactly that which satisfies Eq. (C.12), the first half of the variational principle is proven.

The remaining part of the proof then involves extremizing $D_{n}$ with $\mid f_{c n}>$ held fixed and allowing $\mid f_{s n}>$ to vary. For this purpose we write [from Eq. (C.7)] :

$$
\begin{aligned}
& -D_{n}\left(\lambda<f_{s n}|\Omega| f_{c n}>^{2}+<f_{s n}|C| f_{s n}><f_{c n}|C| f_{c n}>\right) \\
& \left.=\left\langle v_{y} \mid f_{\text {sn }}\right\rangle^{2}<f_{\mathrm{cn}}|\mathrm{C}| \mathrm{f}_{\mathrm{cn}}\right\rangle \text {. }
\end{aligned}
$$

Requiring that $\delta D_{n} / \delta f_{s n}=0$ results in the following equation:

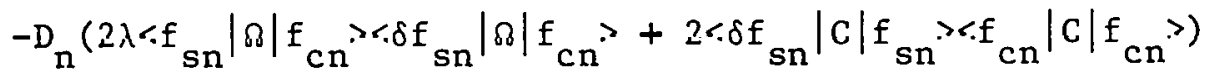

$$
\begin{aligned}
& =2<v_{y}\left|f_{s n}><\delta f_{s n}\right| v_{y}><f_{c n}|c| f_{c n}>
\end{aligned}
$$

Since $\left\langle f_{\mathrm{cn}}|\dot{\mathrm{C}}| \mathrm{f}_{\mathrm{cn}}>=\left\langle\mathrm{f}_{\mathrm{sn}}|\bar{\Omega}| \mathrm{f}_{\mathrm{cn}}>[\right.\right.$ from Eq. (C..3)], Eq. (C.14) reduces to:

$$
<\delta f_{s n}\left|\left[\lambda \Omega\left|f_{c n}\right\rangle+c\left|f_{s n}\right\rangle-\frac{\left\langle v_{y} \mid f_{s n}\right\rangle}{\left(-D_{n}\right)}\left|v_{y}\right\rangle\right]=0,\right.
$$

which is satisfied if: 


$$
\lambda \Omega\left|f_{\mathrm{cn}}>+C\right| f_{s n}>-\frac{<v_{y}\left|f_{s n}\right\rangle}{\left(-D_{n}\right)} \mid v_{y}>=0 .
$$

If Eq. (C.2) is satisfied, then $-D_{n}=\left\langle v_{y} \mid f_{s n}\right\rangle$ and Eq. (C.16) reduces to Eq. (C.2), thus completing the proof. 


\section{REFERENCES}

1. L. Kovrizhnykh, Sov. Phys. - JETP 29, 475 (1969).

2. R. A. Dandl et al., Research Progrom for Plasma Confinement and Heating in ELMO Brompy TOMu Devices, ORNL/TM-4941, Oak Ridge National Laboratory, Oak Ridge, Tennessee (1975).

3. C. L. Hedrick et a1., Nucl. Fusion 17, 1237 (1977).

4. M. N. Rosenbluth and R. D. Hazeltine, Phys. Fluids 15, 116 (1972).

5. F. L. Hinton and M. N. Rosenbluth, Phys. Fluids 16, 836 (1973).

6. J. W. Connor and R. J. Hastie, Phys. Fluids 17, 114 (1974).

7. L. M. Kovrizhnykh, Transport Processes in Toroidal Magnetic Traps, AEC-tr-7274, AEC-tr-7284 (see also Trieste reports IC/70/86 and 70/124).

8. J. G. Cordey and C. J. H. Watson, Fusion Reactor Design Problems, Culham (1974), Nucl. Fusion Suppl., 199.

9. J. G. Cordey and C. J. H. Watson, Proc. IAEA Conf. Plasma Physics and Controlzed Nuclear Research, Tokyo, Vol. II (1974), 643.

10. C. J. H. Watson, The Present Status of Research on Toroidally Linked Mirrors, CLM-P468, Abingdon, Berks, United Kingdom (June 1976).

11. D. A. Spong et al., EBT Transport Coefficients Using ModeZ ColZision Operators, to be published as an ORNL/TM.

12. P. L. Colestock et al., IEEE Intl. Conf. on Plasma Science, New York (1977), papers 1A3, 1 A4.

13. R. D. Hazeltine, Plasma Phys. 15, 77 (1973).

14. E. A. Frieman, Phys. Fluids 13, 490 (1970).

15. B. V. Wadde11, Report LPP-7(MIT), Cambridge, Massachusetts (Ecptcmbcr 1974)\& B. V. Woddell, Nuel. Fusioñ 15, 003 (1975).

16. T. G. Northrop and E. Teller, Phys. Rev. 117, 215 (1960).

17. M. N. Rosenbluth, W. M. MacDonald, and D. L. Judd, Phys. Rev. 107, 1 (1957).

18. D. C. Montgomery and D. A. Tidman, Plasma Kinetic Theory, McGrawHill, New York, 1964.

19. J. Killeen and K. D. Marx, Methods in Computational Physics, Vol. 9, p. 442, Academic Press, 1970. 
20. J. Killeen, A. A. Mirin, and M. E. Rensink, Methods in Computational Physics, Vo1. 16, p. 389, Academic Press, 1976.

21. 0. C. Eldridge, Phys. Fluids 15, 676 (1972).

22. C. L. Hedrick and E. F. Jaeger, Bul1. Am. Phys. Soc. 21, 1070 (1976).

23. E. F. Jaeger and C. L. Hedrick, A Nromerical Model for Radial Transport in the ELMO Bumpy TOrus, ORNL/TM-6013, Oak Ridge National Laboratory, Oak Ridge, Tennessee (1977).

24. A. I. Morozov and L. Solovev, "The Structure of Magnetic Fields," Reviews of Plasma Physics, Vol. 2 (1966), ed. by M. A. Leontovich, Consultants Bureau, New York.

25. B. Robinson and I. B. Bernstein, Ann. Phys. 18, 110 (1962).

26. S. I. Braginskii, "Transport Processes in a Plasma," Reviews of PZasma Physics, Vol. 1 (1965), New York.

27. I. P. Shkarofsky, T. W. Johnston, and M. P. Bachynski, The Particle Kinetics of Plasmas, Chap. 7, Addison Wesley, Reading, Massachusetts, 1966.

28. H. K. Meier, W. I. Van Rij, and C. O. Beasley, Jr., Techniques for Computing the Nromerical Values of the Matrix Elements of the Collision Operator in the Collisional Plasma Model, ORNL/TM-5315, Oak Ridge National Laboratory, Oak Ridge, Tennessee (1976).

29. W. I. van Rij, H. K. Meier, and C. O. Beasley, Jr., Plasma Phys. 19,135 (1977).

30. H. K. Meier, W. I. van Rij, and C. O. Beasley, Jr., Plasma Phys. $\underline{19}, 151$ (1977).

31. C. 0. Beasley, Jr. et al., Hlasma Phys. 19, 593 (1977).

32. J. P. Dougherty, Phys. Fluids I, 317 (1964).

33. W. Magnus, F. Oberhettinger, and R. P. Soni, Formulas and Theorems for tho Sposial. Funstions of Mathemrtinnt. Phynise, Springer=Verlag, New York, 1966.

34. L. D. Landau, Sov. Phys. - JETP 7, 103 (1937).

35. J. D. Lewis, An Expansion Theorem for the Linearized Fokker-Planck Equations, CONF-65-1015-20, Chicago, Illinois. 
ORNL/TM-6215

Dist. Category UC-20g

\section{INTERNAL DISTRIBUTION}

\author{
1. L. A. Berry \\ 2. J. D. Callen \\ 3. R. A. Dandl \\ 4. R. A. Dory \\ 5. G. G. Kelley \\ 6. H. H. Haselton \\ 7. P. N. Haubenreich \\ 8. M. S. Lube11 \\ 9. 0. B. Morgan \\ 10. H. Postma \\ 11. M. W. Rosenthal
}

\author{
12. J. Sheffield \\ 13. D. Steiner \\ 14-47. D. A. Spong \\ 48-49. Laboratory Records Department \\ 50. Laboratory Records, ORNL-RC \\ 51. Document Reference Section \\ 52-53. Central Research Library \\ 54. Fusion Energy Division Library \\ 55. Fusion Energy Division \\ Communications Center \\ 56. ORNL Patent Office
}

\section{EXTERNAL DISTRIBUTION}

57. Bibliothek, Max-Planck Institute für Plasmaphysik, 8046 Garching bei Muinchen, Federal Republic of Germany

58. Bibliothèque, Service du Confinement des Plasmas, C.E.A., B.P. No. 6, 92, Fontenay-aux Roses (Seine), France

59. Lung Cheung, Department of Electronics, University Science Center, The Chinese University of Hong Kong, Shatin, N.T., Hong Kong

60. J. F. Clarke, Office of Fusion Energy, G-234, Department of Energy, Washington, DC 20545

61. R. W. Conn, Fusion Technology Prugram, Nuclear Engineering Department, University of Wisconsin, Madison, WI 53706

62. CTR Library, c/o Alan F. Haughr, United Technologies Research Laboratory, East Hartford, CT 06108

63. CTR Reading Room, c/o Allan N. Kaufman, Physics Department, University of California, Berkeley, CA 94720

64. J. Nar1 Davidson, School of Nuclear Engineering, Georgia Institute of Technology, Atlanta, GA 30332

65. Documentation S.I.G.N., Départment de la Physique du Plasma et de la Fuston Controlée, Association EURATOM-CEA sur la Fusion, Centre d'Études Nucléaires, B.P. 85. Centre du TRI, 38011 Grenoble, Cedex, France

66. W. R. Ellis, Office of Fusion Energy, G-234, Department of Energy, Washington, DC 20545

67. Harold K. Forsen, Exxon Nuclear Co., Inc., 777 106th Avenue, N.E., C-000777, Bellevue, WA 98009

68. Harold P. Furth, Princeton Plasma Physics Laboratory, Princeton University, Forrestal Campus, P.0. Box 451, Princeton, NJ 08540

69. Roy W. Gould, California Institute of Technology, Mail Stop 116-81, Pasadena, CA 91125

70. Robert L. Hirsch, Exxon Research and Engineering, P.0. Box 101, Florham Park, NJ 07932 
71. Raymond A. Huse, Manager, Research and Development, Public Service Gas and Electric Company, 80 Park Place, Newark, NJ 07101

72. T. Hsu, Office of Fusion Energy, G-234, Department of Energy, Washington, DC 20545

73. V. E. Ivanov, Physical-Technical Institute of the Ukranian Academy of Sciences, Sukhumi, U.S.S.R.

74. A. Kadish, Office of Fusion Energy, G-234, Department of Energy, Washington, DC 20545

75. L. M. Kovrizhnikh, Lebedev Institute of Physics, Academy of Sciences of the U.S.S.R., Leninsky Prospect 53, Moscow, U.S.S.R.

76. Fily Lava1, Groupe de Physique Théorique, Ecole Polytechnique, 91 Palaiseau, Paris, France

77. Library, Centre de Recherches en Physique des Plasma, 21 Avenue des Baino, 1007, Lansanne, Switzerland

78. Library, Culham Laboratory, United Kingdom, Atomic Energy Authority, Ahingdon, Oxun, OX14 3DB, United Kingdom

79. Library, FOM-Institut voor Plasma - Fysica, Kijnhulzen, Jutphaas, Netherlands

80. Library, Institute for Plasma Physics, Nagoya University, Nagoya, Japan 464

81. Library, International Centre for Theoretical Phystcs, Trieste, Italy

82. Library, Laboratorio Gas Ionizzati, Frascati, Italy

83. Dsumber G. Lominadze, Academy of Sciences of the Georgian S.S.R., 8 Dzerzhinski St., 38004, Tbilisi, U.S.S.R.

84. Oscar P. Manley, Office of Fusion Energy, G-234, Department of Energy, Washington, DC 20545

85. D. G. McAlees, Exxon Nuclear Co., Inc., Research and Technology Laser Enrichment Department, 2955 George Washington Way, Richland, WA 99352

86. J. E. McCune, School of Engineering, Department of Aeronautics and Astronautics, Bldg. 37-391, Massachusetts Institute of Technology, Cambridge, MA 02139

87. Claude Mercier, Service du Theorie des Plasmas, Centre d'Études Nucléaires, Fontenay-aux-Roses (Seine), France

88. K. G. Moses, Office of Fusion Energy, G-234, Department of Energy, Washington, DC 20545

89. D. Pfirsch, Institute for Plasma Physics, 8046 Garching bei München, Federal Republic of Germany

90. Plasma Physics tròup, Depidttment of Enginepring Physics, Australian Natiulal Inivereity, P.n. Box 4, Canberra A.C.T. 2600, Australia

91. Robert E. Price, Office of Fusion Energy, G-234, Department of Energy, Washington, DC 20545

92. A. Rogister, Institute for Plasma Physics, KFA, Postfach 1913, D-5170, Jülich 1, Federal Republic of fermany

93. W. Sadowski, Office of Fusion Energy, G-234, Department of Energy, Washington, DC 20545

94. V. D. Shafranov, I. V. Kurchatov Institute of Atomic Energy, 46 Ulitsa Kurchatova, P.0. Box 3402, Moscow, U.S.S.R. 
95. Yu. S. Sigov, Institute of Applied Mathematics of the U.S.S.R. Academy of Sciences, Miuskaya, Sq. 4, Moscow A-47, U.S.S.R.

96. W. M. Stacey, Jr., School of Nuclear Engineering, Georgia Institute of Technology, Atlanta, GA 30332

97. J. B. Taylor, Culham Laboratory, U.K. Atomic Energy, Authority, Abingdon, Oxon, OX14 3DB, United Kingdom

98. Thermonuclear Library, Japan Atomic Energy Research Institute, Tokai, Naka, Ibaraki, Japan

99. Francisco Verdaguer, Director, Division of Fusion, Junta de Energia Nuclear, Madrid 3, Spain

100. Director, Research and Technical Support Division, Department of Energy, Oak Ridge Operations, P.O. Box E, Oak Ridge, TN 37830

101-276. Given distribution as shown in TID-4500, Magnetic Fusion Energy (Distribution Category UC-20g, Theoretical Plasma Physics) 